\title{
TRANSIT SIGNAL PRIORITY WITH CONNECTED VEHICLES TECHNOLOGY
}

\section{Dissertation Defense}

Jia $\mathrm{Hu}$

\author{
Dissertation Committee \\ Advisor: Byungkyu (Brian) Park \\ Brian L. Smith \\ John S. Miller \\ Michael D. Fontaine \\ Andrew Mondschein
}

June 13, 2014 


\section{ACKNOWLEDGEMENT}

I wish to convey my sincere appreciation for those who have supported me in successfully completing my doctoral study. I would like to first express my deepest gratitude to my advisor, Dr. Byungkyu (Brian) Park, for his assistance in all my research questions and concerns. This dissertation would not have been possible without his guidance and support. He has always led me to the right path of being a better researcher.

I would like to sincerely thank the members of my dissertation committee as well: Dr. Michael Fontaine, Dr. John Miller, and Dr. Brian Smith in the Department of Civil and Environmental Engineering, and Dr. Andrew Mondschein in the Department of Urban and Environmental Planning, for their efforts and feedback on this dissertation.

I also give my appreciation to everyone who has supported me, and my particular thanks to Dr. Noah Goodall, Dr. Joyoung Lee, Dr. Young-Jae Lee, Mr. Peter Ohlms, Dr. Emily Parkany, Mr. Amit Sidhaye, Dr. Jaehyun(Jason) So and Dr. Yingjie Xia for their sincere help on my research project and this dissertation.

I would like to take this opportunity to thank Dr. WeiLiang Jin from my undergraduate and Dr. George List and Dr. Nagui Rouphail from my master's study. I could never have reached the heights or explored the depths without their help and guidance.

A very special thank-you to my friends Long Chen, Long Di, Xiaochen Huang and Hantao Zhong for the support they have lent me over all these years. Thanks a lot for everything!

Lastly, I wish to thank my parents, Hongbo Hu and Jianyue Dai, and my dear one for their unconditional love and support. 


\begin{abstract}
Transit Signal Priority (TSP) has been proposed and studied as a control strategy that offers preferences to transit vehicles at signalized intersections. Conventionally, many challenges have been identified that are preventing the TSP to be widely deployed, for example, adverse effect on side streets, and uncertainty of benefit. Closer investigation on these challenges reveals that these shortcomings are mainly caused by the fact that the logic of conventional TSP is based on data collected from the past instead of the present. If with real time data, many uncertainties can be eliminated, and correspondingly, TSP could perform better with higher reliability.
\end{abstract}

The emerging new system known as connected vehicles is able to feed TSP with present data and also create many other possibilities for the TSP. In a connected vehicles environment, diagnostic sensors are installed on every vehicle to collect data and data are being transmitted wirelessly between vehicles and nearby infrastructures. It no longer has to rely on conventional data collection equipment, like loop detector or video detections, and it collects much more information than the conventional ways. Measurements that are previously unknown are now available, which include but not be limited to: vehicle speeds, positions, arrival rates, rates of acceleration and deceleration, queue lengths, stopped time and so on.

A system of bus priority techniques is developed, taking advantages of the resource provided by Connected Vehicles (CV) technology, including two-way communications between the bus and the traffic signals, accurate bus location detection and prediction, and other information. The TSP logics allow cooperative control that traffic signal and transit bus work together. The cooperation requires a bus to travel at a reasonable speed which is recommended based on road geometry, normal signal timing plan and remaining/expected queue. The TSP strategy used is the green time reallocation, which only moves green time instead of adding extra green time. The TSP is also designed to be conditional on certain criteria. Delay per person is used as one of the most important criterion to decide whether TSP shall be granted. The developed TSP techniques are able to accommodate: single TSP request at an isolated intersection, bus merging at a nearside bus stop, multiple conflicting TSP requests at an isolated intersection and bus progression along a corridor.

The logic developed in this research is evaluated in two ways: with analytical and microscopic simulation approaches. The proposed TSP techniques are usually compared against two scenarios: no TSP and conventional TSP. The measures of effectiveness (MOE) used are bus delay and per person delay of all travelers. Simulation-based evaluation results show that, compared to conventional TSP, the proposed TSP logic reduces bus delay of a single TSP request between $9 \%$ and $84 \%$, minimizes bus delay during conflicting requests between $5 \%$ and $48 \%$, decreases delay of buses progressing along a corridor between $35 \%$ and $68 \%$, and cut back up to $30 \%$ of delay buses lose at merging from nearside bus stops. The range of improvement corresponding to the four different $\mathrm{v} / \mathrm{c}$ ratios tested, which are $0.5,0.7,0.9$ and 1.0. In most cases, no significant negative effects are caused by the proposed TSP logic. 


\section{Table of Contents}

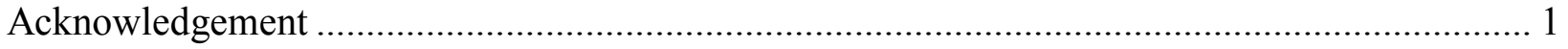

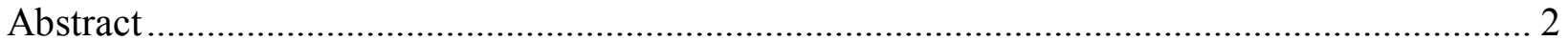

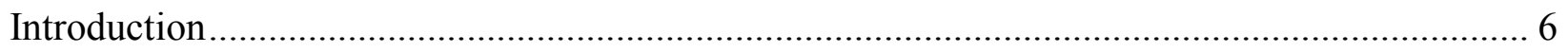

Transit Signal Priority with Connected Vehicle Technology ......................................................... 10

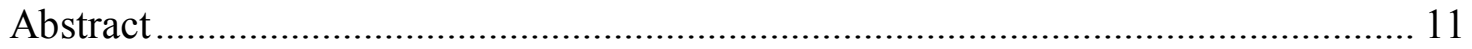

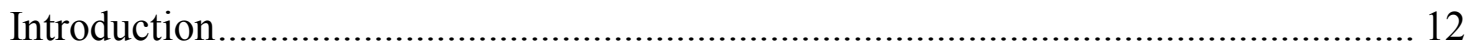

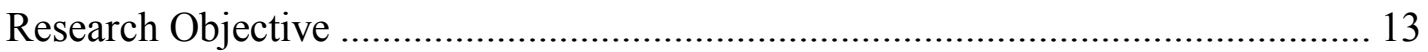

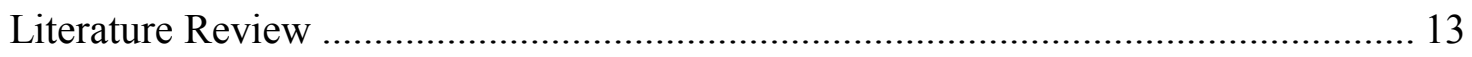

Conventional TSP Logic .................................................................................... 13

State of the Art TSP for One Bus Scenario............................................................... 14

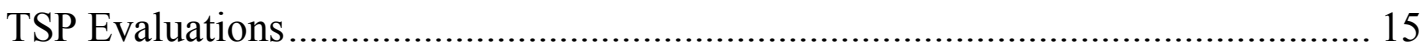

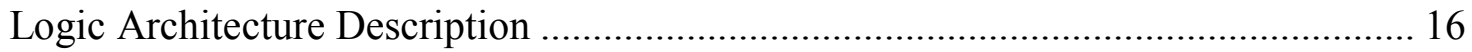

Arrival Time Prediction Component................................................................... 17

TSP Timing Plan and Bus Speed Calculation Component ........................................ 17

Logic Assessment and Implementation Component................................................ 19

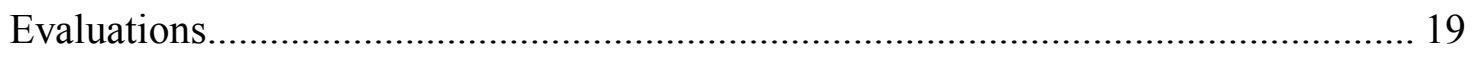

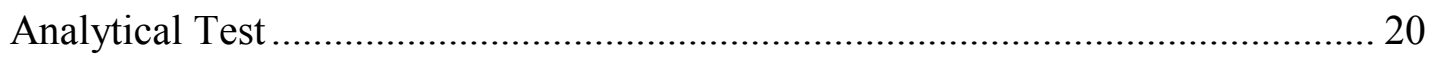

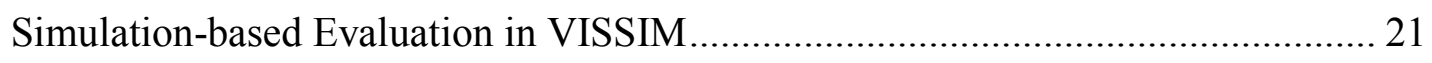

Sensitivity Analysis on Congestion Levels............................................................. 22

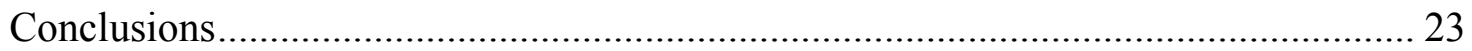

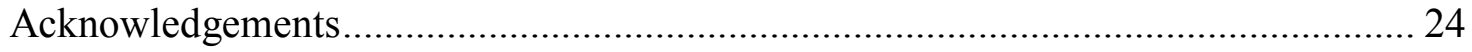

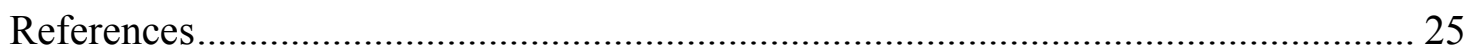

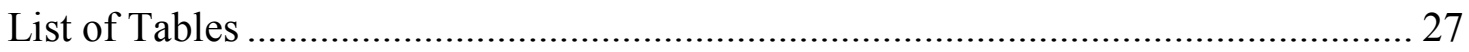

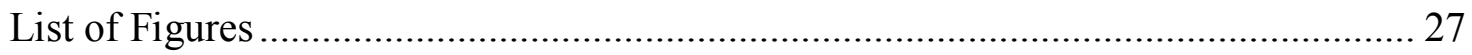

Near-Side Bus Stop with Queue Jumper Lane under Connected Vehicles ................................. 32

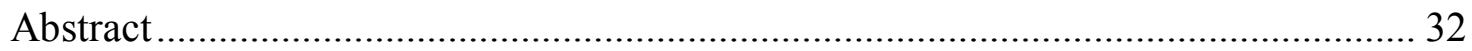

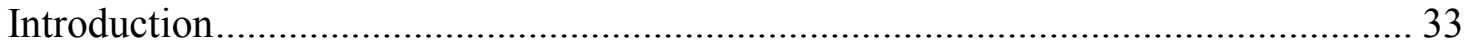

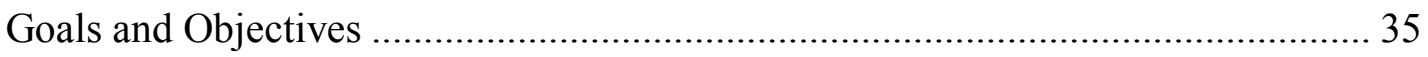

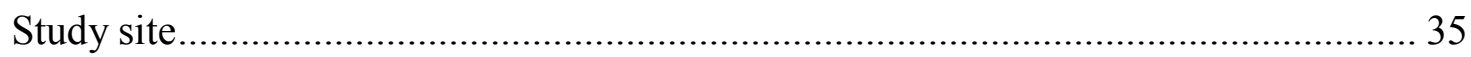

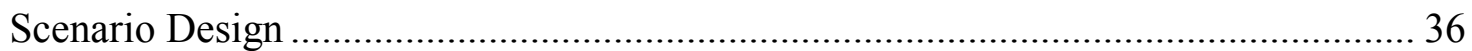

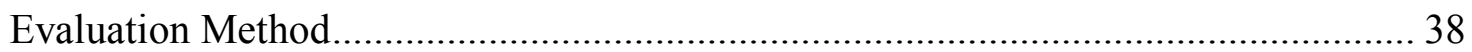

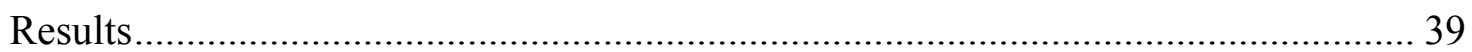

Congestion Level ......................................................................................... 39

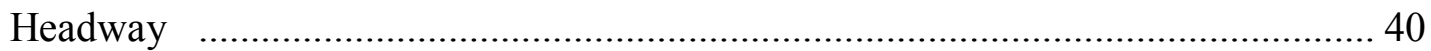


Right Turn Volume ............................................................................................ 42

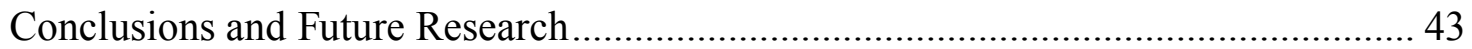

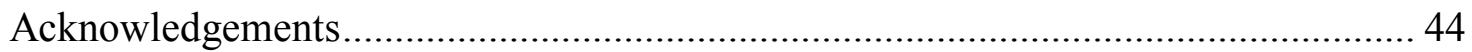

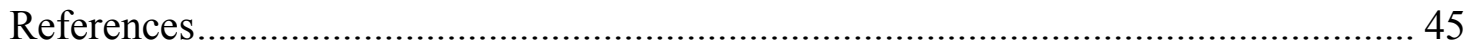

Intelligent Transit Signal Priority Considering Conflicting Requests ..................................... 47

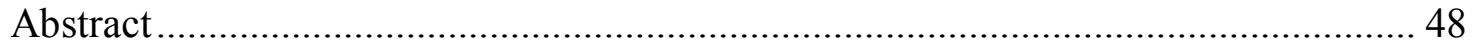

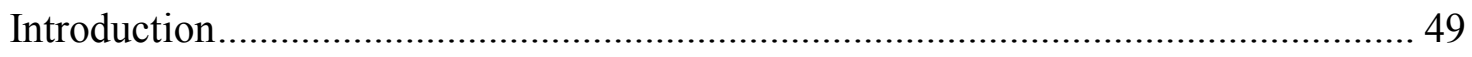

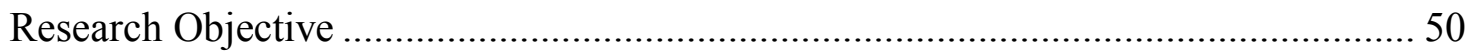

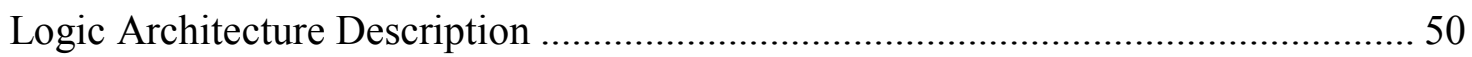

Arrival Time Prediction Component........................................................................... 51

TSP Timing Plan and Bus Speed Calculation Component ........................................ 51

Logic Assessment and Implementation Component ................................................ 54

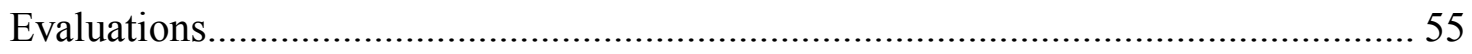

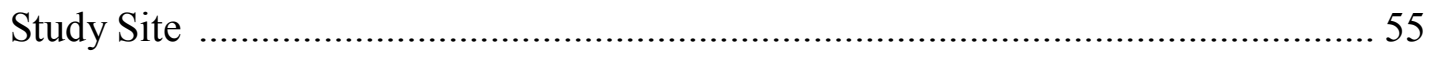

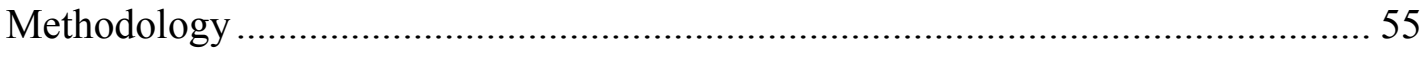

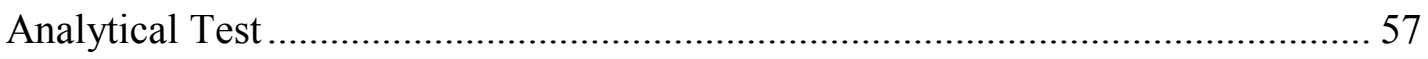

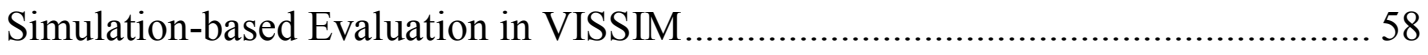

Conflicting Requests From Opposite Directions ……………………………......... 59

Conflicting Requests from Perpendicular Directions .................................................... 60

Conflicting Requests from Three Directions ........................................................... 61

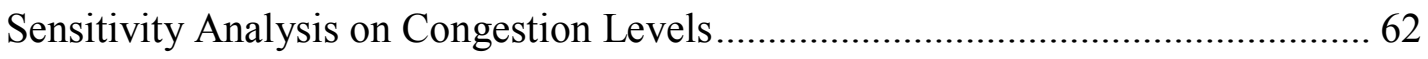

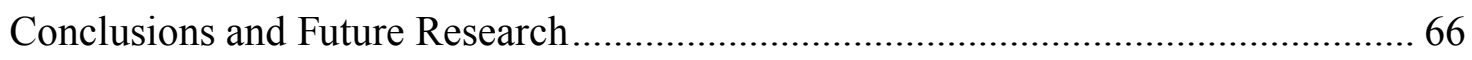

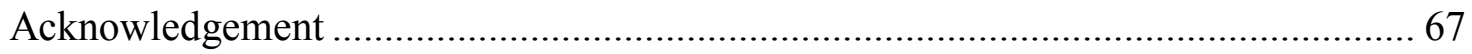

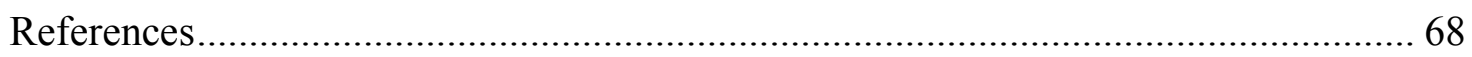

Intelligent Transit Signal Priority Considering Bus Progression.................................................. 70

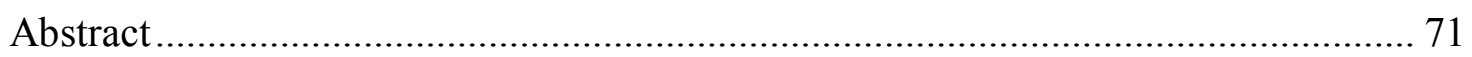

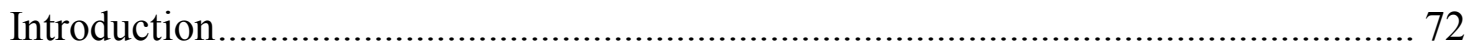

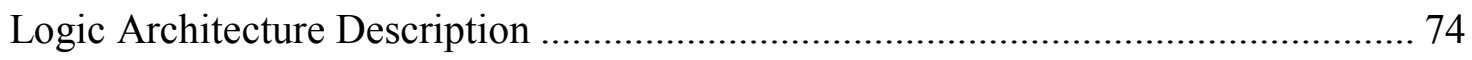

Arrival Time Prediction Component.................................................................. 76

TSP Timing Plan and Bus Speed Calculation Component ......................................... 76

Logic Assessment and Implementation Component.................................................. 78

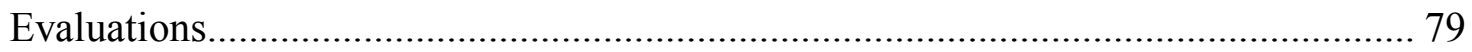

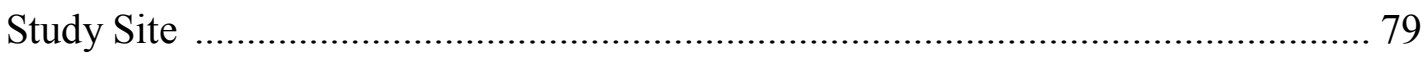

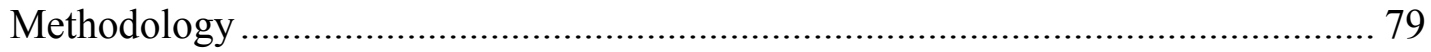




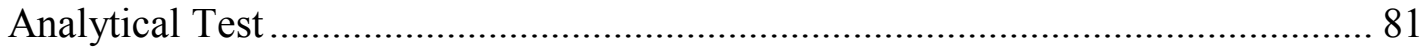

Simulation-based Evaluation in VISSIM ......................................................... 82

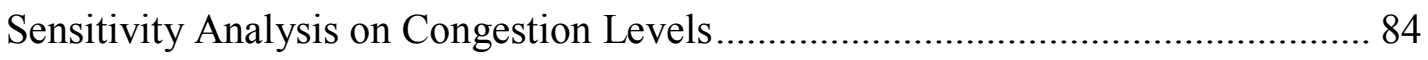

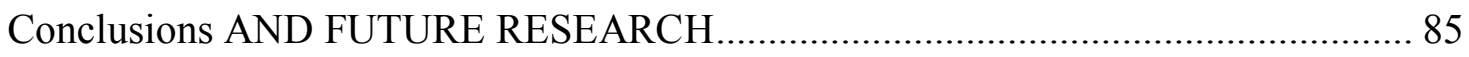

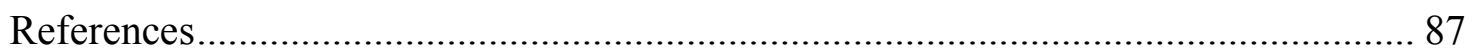

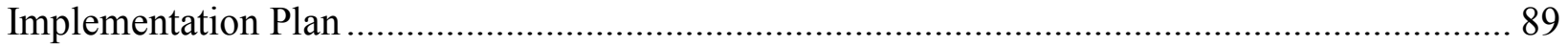

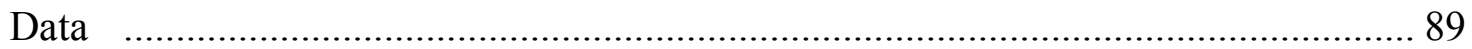

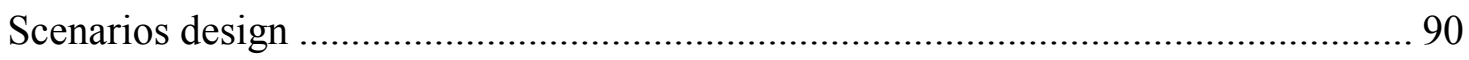

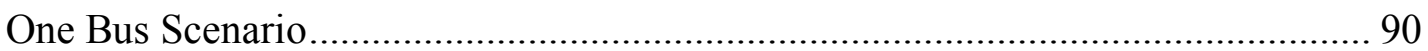

Multiple Conflicting Requests ................................................................... 90

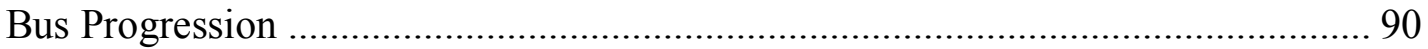

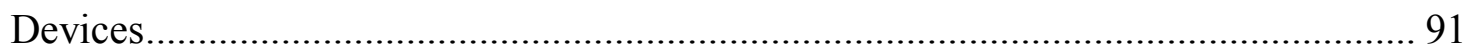

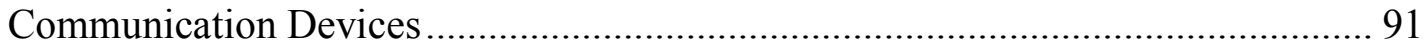

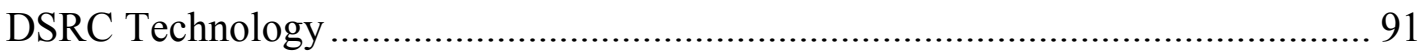

Commercial Cellular Services ........................................................................ 91

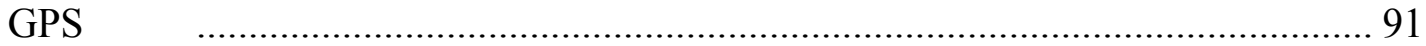

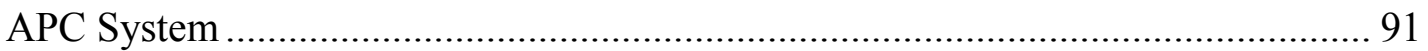

Blue Tooth Reader ........................................................................................ 91

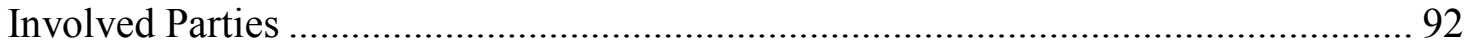

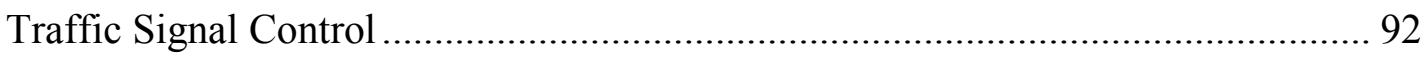

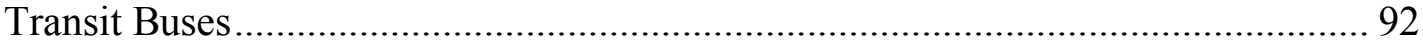

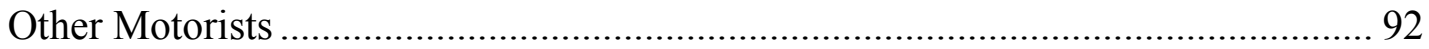

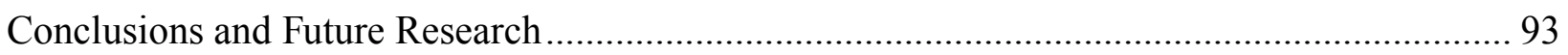

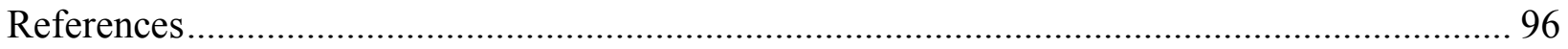




\section{INTRODUCTION}

Congestion is becoming more serious every day around the world, especially for cities with growing population, where demand is rising while road geometry is constrained by existing architects. To solve this dilemma, one solution is to promote public transportation. By increasing passenger density, the throughput and capacity of an existing road would be increased. Transit bus, as a public transportation mode, is presently being utilized in most metropolitan areas. By nature, transit bus is designed to efficiently move large amount of passengers through areas with dense population. However, current transit bus system has its shortcoming compared to private transportation, for example, longer travel time, less reliable and worse comfort. To draw more riders to the public sector, it is important to improve ridership quality of transit buses. Therefore, many believe that preference should be placed on transit buses at signalized intersections. By having signal plan adjusted according to bus arrival, the delay that transit buses spend at intersections would be reduced, as a result, travel time can be saved and transit quality of service can be increased. This action of giving preference to transit buses is often referred to as Transit Signal Priority (TSP).

However, conventional TSP shows a lot of shortcomings and has considerable room to be improved. Conventionally, TSP is activated when a transit bus sends out a request for TSP when it is approaching an intersection from upstream. In most of the cases, the strategy of TSP is simple extension or early start of its original green time. As for which strategy should be used, a quick calculation is performed on site driven by a bus arrival time model fed with historical data. If the bus is expected to arrive shortly before its original green time, the green time starts early, if the bus is expected to arrive shortly after its original green time, the green time is extended.

This kind of the TSP logic has its restriction in many ways. Most importantly, because the data fed into the model is out dated, the bus arrival time forecast could be severely biased. The false forecast of bus arrival time could lead to the waste of extra TSP green time and cause unnecessary but tremendous adverse effect of side streets. Besides, even with extension and early start, conventional TSP can only cover a small portion of a signal cycle, therefore, a large portion of buses could not benefit from employing conventional TSP. Apart from that, conventional TSP rarely deals with conflicting TSP requests of more than 2 buses. And up to now, very few studies were found researching resolve conflicting TSP calls. Nevertheless, Zlatkovic et al. pointed out in his paper that the conventional "first come first serve" way of dealing with conflicting TSP request is very inefficient. He proved his argument by proposing a very simple logic which, in short, always provides priority first to the direction with green phase on (1). Compared to the conventional "first come first serve", this simple logic already shows a benefit of more than $30 \%$ reduction on traffic delay.

The problems that the conventional TSP bears could be solved by introducing the technology usually referred to as "Connected vehicles". This technology put diagnostic sensors onto vehicles and has data collected been transmitted wirelessly between vehicles and nearby infrastructures. It no longer needs to rely on conventional data collection equipment, like loop detector or video 
detections, and it collects much more information than the conventional approaches.

Measurements that are previously unknown are available, which include but not be limited to: vehicle speeds, positions, arrival rates, rates of acceleration and deceleration, queue lengths, and stopped time and so on.

With this extra information, many applications are made possible. These applications are usually categorized into 3 major kinds: improve safety, enhance mobility and minimize environmental impact. It is important to understand that not all applications share the same interest from state and local transportation agencies, but TSP with connected vehicles technology (TSPCV) is one of the connected vehicles applications that best serve the interests of transportation agencies and their constituents. According to the AASHTO Connected Vehicles Infrastructure Deployment Analysis (2), TSPCV is one of the key applications that could enhance mobility. It is also confirmed by the USDOT which include TSPCV in its list of High-Priority Dynamic Mobility Applications (3).

TSPCV is worth the attention also because it is one of the few applications that generate benefits even at the early stage of connected vehicles system rollout. By nature, transit buses are typical good targets for equipping highly customized electronic devices. According to 2006 data, the number of transit buses serving in the United States is around 70,000 (2). Therefore, the cost of investing on TSPCV is relatively modest compared to other applications which require almost ubiquitous coverage for effectiveness. Let alone the cost, it would still take time for connected vehicles market penetration reaching an optimum level. It is forecasted by AASHTO research team that at least 10 years is necessary for connected vehicles to reach $90 \%$ of market penetration (2). This is even an optimistic forecast, given the FHWA predicts 9 years for market rates to reach $50 \%$ and 30 year to $90 \%$ (4). The growth rate follows an S-Curve which means low initial growth, max middle years growth and flatter growth in later years. In other words, a long period of time shall we observe for connected vehicles market rate rise above $50 \%$ and that is under the assumption of mandatory. In the worst case, if without mandatory, market rate could possibly level off before it reaches $90 \%$. This is exactly the case with the Anti-Lock Brake System (2).

The importance of researching TSPCV also lies in the fact that it could be the foundation of ecodriving with connected vehicles technology. This technology, sometimes also referred to as dynamic eco driving, provides real time driving advice to motorists on both freeway and arterials based on real time traffic condition and signal phase information. For example, on an arterial, if the driver learns whether he can make through the next intersection, he would be able to decide whether he should keep driving or gradually decelerate. Compared to hitting the brakes at the light, motorists save fuel and reduce emission. This is just one example of eco driving; there are a lot more possibilities to be explored, while very limited research has been performed.

Although the importance of TSP with connected vehicles (referred to here as TSPCV) has been identified by most agencies, the mechanism of how TSPCV works is still undefined to all of 
them. The clearest definition of TSPCV is found in an updated description on USDOT HighPriority Dynamic Mobility Applications (3). This guideline proposes, by equipped with on-board equipment, transit vehicles should be able to communicate information such as passenger count data, service type, scheduled and actual arrival time and heading information to roadside equipment via DSRC. Clearly, this definition is not sufficient for field implementation.

Therefore, in this research, the researcher developed a collection of TSPCV logics that fully realizes the connected vehicles technology based on two-way communications among multiple transit buses and traffic signal, and among the transit buses and vehicles. This next generation Transit Signal Priority (a.k.a. TSPCV) no longer has to rely on conventional TSP sensors. This is because $\mathrm{CV}$ technology provides better real-time information about bus location, and passenger count to support more TSP possibilities. One can expect that with better data, bus arrival time could be predicted with higher accuracy. Therefore, with the help of CV technology, the logic of TSP becomes more flexible instead of simple "green extension" and "red truncation." The CVbased logic grants extra TSP green time more preciously to where it is most needed. The less waste of extra TSP green time, the less adverse side streets effect would be.

This research also upgrades TSP in terms of bus-signal cooperation. Because 2-way communication is made possible by DSRC, transit buses not only are able to "tell" the signal where it is, but also get instructions (ex. Speed) from the signal. Hence, in contrast to the current TSP logic where a bus (or buses) approaching at an intersection sends priority request and the traffic control tries to accommodate it, this logic requires cooperation from the bus as well. The cooperation requires a bus to travel at a reasonable speed which is recommended based on road geometry, normal signal timing plan and remaining/expected queue. The TSP strategy used is the green time reallocation, in other word, instead of adding additional green time to the original timing plan this TSP logic splits the original green time and moves part of it to when green time is most needed by a transit bus (or buses). Furthermore, in addition to the schedule adherence, the logic accounts for delay per person as a criterion to grant the TSP green time. The delay per person measurement indicates the trade-off between travel time saved by bus and travel time wasted by side streets. Therefore, the strategy is expected to have more control on the adverse side effect than basic "green extension" and "red truncation" kind of TSP. In this way, we take advantages of the resource provided by Connected Vehicles (CV) technology, including two-way communications between the bus and the traffic signal, accurate bus location detection and prediction, and other extra information.

The remainder of this dissertation is arranged in journal paper format. Each paper covers one aspect or scenario in terms of TSP with connected vehicles.

The first paper proposes a TSPCV logic designed to accommodate single TSP request at an isolated intersection. This TSP technology takes advantage of the two-way communications and additional and more accurate information provided by Connected Vehicle technology. The paper has been accepted for publication by the Journal of the Transportation Research Record. 
The second paper demonstrates a bus priority technique that helps transit buses merge back into traffic from nearside bus stops and reduces delay associated. The paper has been accepted for presentation by the $21^{\text {st }}$ World Congress on Intelligent Transport Systems.

The third paper presents a method concerning the resolving of 2 or more conflicting TSP requests. Research has shown that the conventional approach of granting TSP to whichever bus comes first is a very inefficient way of distributing green time. With more accurate information of speed and location of every traffic users, the researcher is aiming to find a best way to distribute green time to buses coming from different directions and cause the minimum overall delay.

The last paper tackles the problem of the coordination of TSPCV between adjacent intersections. The research provides methods to secure the mobility benefit generated by intelligent TSP logic along a corridor so that the bus delay saved at one intersection is not wasted at the downstream intersections. 
By

\author{
$\mathrm{Jia} \mathrm{Hu}^{*}$,1) \\ Ph.D. Candidate \\ Tel: 919-744-9842, Email: jh8dn@virginia.edu \\ Byungkyu "Brian" Park, Ph.D. ${ }^{1)}$ \\ Associate Professor \\ Tel: (434) 924-6347, Email: bpark@virginia.edu \\ A. Emily Parkany, Ph.D. ${ }^{1}$ \\ Associate Director, Center for Transportation Studies \\ Tel: (434)-982-2897, Email: aep5x@,virginia.edu \\ 1) Department of Civil and Environmental Engineering \\ University of Virginia \\ P.O. Box 400742 \\ Charlottesville, VA 22904-4742 \\ Fax: (434) 982-2951
}

November 2013

Submitted for consideration for publication and presentation at the $93^{\text {rd }}$ Annual Meeting of the Transportation Research Board, January 12-16, 2014

Word Count: 5,996 text words plus 1,500 for figures/tables $(6 * 250)=7,493$ total

*Corresponding Author 


\section{ABSTRACT}

Transit Signal Priority (TSP) has been studied as a control strategy that offers preferences to transit vehicles at signalized intersections. While TSP has been deployed in many places, several shortcomings such as adverse effect on side streets and uncertainty of benefit have been identified. Therefore, a new Transit Signal Priority (TSP) logic is proposed to overcome these shortcomings, taking advantage of the resources provided by Connected Vehicle (CV) technology, including two-way communications between the bus and the traffic signal controller, accurate bus location detection and prediction and the number of passengers. The key feature of the proposed TSP logic is green time re-allocation, which moves green time instead of adding extra green time. The TSP is also designed to be conditional. That is, delay per person is used as the most important criterion deciding whether TSP is to be granted.

The logic developed in this research is evaluated in two ways: with analytical and microscopic simulation approaches. In each evaluation, the proposed TSP is compared against two scenarios: no TSP and conventional TSP. The measures of effectiveness used are bus delay and per person delay of all travelers. Simulation-based evaluation results show that the proposed TSP logic reduces bus delay between $9 \%$ and $84 \%$ compared to conventional TSP and between $36 \%$ and $88 \%$ compared to the no TSP condition. The range of improvement corresponding to the four different $\mathrm{v} / \mathrm{c}$ ratios tested, which are $0.5,0.7,0.9$ and 1.0. No significant negative effects are caused by the proposed TSP logic. 


\section{INTRODUCTION}

Transit bus, as a transportation mode, is presently being utilized in almost every city around the world. By nature, transit bus is designed to efficiently move large numbers of passengers through areas with dense population. Because of this, many believe that preference should be given to transit buses at signalized intersections. By adjusting the traffic signal plan according to bus arrivals, the delay that transit buses experience at intersections would be reduced, and therefore, travel time can be saved and transit service quality can be improved. This action of providing preference to transit buses is referred to as Transit Signal Priority (TSP).

Conventionally, TSP is activated when a transit bus sends out a request as it approaches a traffic signal controlled intersection. In most cases, the logic of TSP is a simple extension to or early start of the originally planned green time. To decide which logic should be used, a quick calculation is performed on site using a bus arrival time model based on historical data. If the bus is expected to arrive shortly before the original green time, the green time starts early; if the bus is expected to arrive shortly after the original green time ends, the green time is extended. This kind of TSP logic is restricted in many ways. Most importantly, because the data fed into the model is either outdated or not accurate, the bus arrival time forecast could be severely biased. The inaccurate forecast of bus arrival time could lead to the waste of extra TSP green time and cause unnecessary adverse effects on side streets. Additionally, even with extension and/or early start, conventional TSP green time can only cover a small portion of a traffic signal cycle, therefore, a large portion of buses may not benefit from the TSP.

To properly address these challenges, a more sophisticated algorithm which would provide service to a greater proportion of transit buses as needed. This might not be easily accomplished by using inaccurate and outdated data collected from conventional sensors, such as loop detectors or video cameras. Therefore, it is necessary to strengthen conventional TSP with the new emerging "Connected Vehicle" technology. This technology puts diagnostic sensors onto vehicles and collects data transmitted wirelessly between vehicles and nearby infrastructure. Instead of relying on conventional data collection equipment, it collects more accurate information. Additional measurements that were previously not available include vehicle speeds, positions, arrival rates, rates of acceleration and deceleration, queue lengths, number of passengers and stopped time.

With this extra information, many applications would be possible. These applications are usually categorized into improving safety, enhancing mobility and minimizing environmental impact. It is important to understand that not all applications share the same priority among State and local transportation agencies, but TSP with Connected Vehicle (TSPCV) is one of the Connected Vehicle applications that best serve the interests of transportation agencies and their constituents. According to the AASHTO Connected Vehicle Infrastructure Deployment Analysis [1], TSPCV is one of the key applications that would enhance mobility. USDOT also includes TSPCV in its list of High-Priority Dynamic Mobility Applications [2].

It takes time (i.e., sizable market penetration rate) for Connected Vehicle systems to fully realize their potential, but TSPCV is one of the few applications that would generate benefits even at the early stage of a Connected Vehicle system rollout. By nature, transit buses are typically good targets for equipping highly customized electronic devices. According to 2006 data, the number of transit buses deployed in the United States is around 70,000 [1]. Therefore, 
the cost of investing in TSP would be relatively modest compared to other applications which may require almost ubiquitous coverage to realize sizable benefit. Putting aside the cost, it will take time for Connected Vehicle to reach a certain level of market penetration. The AASHTO research team forecasts that at least 10 years is necessary for Connected Vehicle to reach $90 \%$ market penetration [1]. This is an optimistic forecast, given that the FHWA predicts 9 years for market rates to reach $50 \%$ and 30 years to reach $90 \%$ [3]. The growth rate would follow an SCurve which means low initial growth, max middle years growth and flatter growth in later years. In other words, a long period of time is expected before the Connected Vehicle market rate rises above $50 \%$ and that is under the assumption of mandatory devices in new cars. In the worst case, without a mandate, the market rate could possibly level off before it reaches $90 \%$. This is exactly the case with Anti-Lock Braking Systems [1].

Although the importance of TSP with Connected Vehicle (TSPCV) has been identified by most agencies, the mechanism of how TSPCV works is yet to be determined. An up-to-date definition of TSPCV is found in "Updated description on USDOT High-Priority Dynamic Mobility Applications" [2]. This guideline proposes, by equipping with on-board equipment, transit vehicles should be able to communicate information such as passenger count data, service type, scheduled and actual arrival time and heading information to roadside equipment via Dedicated Short Range Communications (DSRC). Unfortunately, this definition is not sufficient for field implementation. This paper describes a potential field implementation of TSPCV.

\section{Research Objective}

Therefore, this research would present a new TSP method that will fully utilize Connected Vehicle technology based on two-way communications between transit buses and traffic signals, and among transit buses and vehicles. This next generation Transit Signal Priority (TSPCV) would not have to rely on conventional TSP sensors. This is because CV technology will provide better real-time information of bus location and number of passengers to support the proposed TSP logic. It is expected that with better data, bus arrival time could be predicted with higher accuracy. Therefore, with the help of CV technology, the logic of TSP could be more flexible instead of simple "green extension" or "red truncation." The CV-based logic will grant extra TSP green time more precisely to where it is most needed. The less waste of extra TSP green time, the fewer adverse side street effects it would cause.

The remainder of this paper reviews the relevant literature, explains the need for research, states the rationale of TSPCV, presents analytical and simulated test results and findings, and identifies the contributions.

\section{LITERATURE REVIEW}

This literature review was undertaken to understand the need to research this area and to understand the state-of-the-art of TSP logic. This section organizes the discussion of literature into three sections: conventional TSP logic, state-of-the-art TSP logic for a one-bus scenario, and TSP evaluation.

\section{Conventional TSP Logic}

For years, Transit Signal Priority has been proposed and studied as an efficient way of improving transit operations. It provides preference to transit at traffic signalized intersections and has potential in reducing transit travel time and improving schedule adherence and customer ride 
quality. Furthermore, it has been discovered that TSP has the ability to cancel out some of the adverse effects due to outdated timing plans [4]. The technology has been applied in many cities in Europe, Asia and North America. In the US, Seattle, Portland, Los Angeles, Chicago and several other large cities have all implemented conventional TSP systems [5].

However, conventional TSP has shortcomings which have limited the promotion of TSP. One big disadvantage is its adverse effect on side streets. Especially for intersections that are nearly operating at their capacity, the benefit of adding TSP is controversial [6]. Another potential challenge of the current TSP is the great uncertainty in bus arrival time prediction. As a result, longer green time is taken from side streets to ensure that bus can pass the intersection during TSP green. This increases adverse effects on traffic.

In Canada, a project team consisting of professionals from a wide range of municipalities representing both traffic and transit agencies once developed a list of issues related to TSP [7]. In the US, transportation and emergency personnel from the Washington D.C area have identified questions and reservations against the deployment of TSP systems [8]. Together, a list of needs, issues and concerns related to TSP was made to guide this research. The list is presented as follows:

- A balance needs to be maintained between transit and non-transit users. It is the goal to reduce transit signal delay, but it is also important to consider the impact on the side streets.

- Minimizing transit travel time to improve schedule flexibility and reduce operation cost.

- Improvement on schedule adherence is as important as travel time saving.

- Bus priority system should be included in a larger ITS system with improved rider information.

\section{State of the Art TSP for One Bus Scenario}

To address the shortcomings of conventional TSP technology, research efforts have been dedicated to find advanced TSP logic. Advanced TSP, also known as intelligent TSP, improves the logic using three essential aspects: arrival time prediction module, TSP logic library and selective priority.

For the arrival time prediction module, unlike conventional TSP which uses fixed location check-in and check-out detectors [6], intelligent TSP takes advantage of AVL systems that are usually equipped on many transit buses today [5][9] [10][11][12]. The upgraded AVL hardware does improve the travel time prediction in between bus stops while it is still unable to help predict dwell time at the bus stops.. In addition to hardware upgrades, Lee et al. [9] took TSP one step further and proposed to use a high-performance online microscopic simulation model for the purpose of predicting transit travel time. But the complexity of this model makes it impractical for a real-time application. Nevertheless, not all prediction models are so complicated, for example, Ekeila et al. [11] utilized a simple linear model based on historical data. But the simplicity could result in the sacrifice of accuracy.

A number of new TSP strategies have been added to the TSP logic library. Apart from the basic TSP strategies which are "green extension," "red truncation" and "phase skipping" [9], green time extension at other phase transitions is made possible with AVL [10], cycle extension is proposed and found to be beneficial during rush hours [11], compensation is introduced to 
limit the adverse effects on the side streets by cutting or skipping the time from the non-bus phase, and finally combining TSP consideration into adaptive signal control [5] [12]. All of these strategies aim for one goal: maximizing the benefit of TSP while minimizing the negative impact on side streets. But so far, restricted by the limited information regarding bus passenger and other motorists, more precise strategy like green re-allocation has not been investigated.

Selective priority is also an enhancement to TSP that is meant to reduce adverse effects on non-transit users. Balke et al. [10] and Skabardonis [13] proposed that TSP logic should consider selective priority that grant priority only to transit buses that meet certain requirements, for example, deviation from schedule or average headway. This kind of TSP requires additional mechanisms to determine if the bus meets the criteria. The mechanism will make sure that no unnecessary delay is added to non-transit users, for instance, if the bus is on schedule. A secondary benefit of granting selective priority is less transit travel time variability. Unreliability in service can increase uncertainty and anxiety among passengers [14]. By improving schedule adherence, better public transportation service would be achieved. A simulation study on conditional TSP shows that selective priority would bring statistically significant improvements of $3.2 \%$ in bus service reliability and $0.9 \%$ for bus travel time [15]. Christofa [16] and Mirchandani et al. [17] proposed to condition TSP based on delay of all traffic users. This rationale of giving higher weight to transit bus, instead of providing bus with absolute priority shares some similarity with this research. However, closer investigation of Christofa's and Mirchandani et al.'s logic revealed that their control algorithm is different from this paper and they did not take advantage of other CV technology features including two-way communications between bus and signal facility.

It is noted that there is no completed research strengthening TSP with Connected Vehicle technology. Currently, there is one ongoing project that aims at designing a multi-modal intelligent traffic signal system that would operate in a Connected Vehicle environment - the Multi-Modal Intelligent Traffic Signal System (MMITSS) project [18]. This MMITSS project investigates TSP at a high level without developing actual algorithms under a connected vehicle environment.

\section{TSP Evaluations}

The benefits of implementing TSP vary significantly from site to site. Table 1 summarizes TSP benefits and disbenefits from various research efforts. The travel time savings could be as low as $2 \%$ [10] or as high as $71 \%$ [12]. Therefore, it is necessary to perform an evaluation of new TSP logic at specific sites before implementation. Although TSP has been installed in many cities, it appears that very few deployments were followed up and evaluated after installation. The most common way to evaluate TSP logic is through simulation, only one out of 13 studies investigated performance benefits based on field testing [19]. Various simulation models have been utilized in the past: PARAMICS [9], AIMSUN [5], TexSIM [10], VISSIM [11] and [13], NETSIM [4] and [12] and WATSim [6]. 


\section{LOGIC ARCHITECTURE DESCRIPTION}

Based on the background information collected, it is clear that several aspects can be improved for the current TSP strategies. First, combining TSP with Connected Vehicle technology is believed to be beneficial. This is because $\mathrm{CV}$ technology may provide better real-time information of bus location and number of passengers to support better TSP performances. Second, with the help of CV technology, the logic of TSP could be more flexible instead of simple "green extension" and/or "red truncation." The CV-based logic will grant extra TSP green time more precisely to where it is most needed. The less waste of extra TSP green time, the less adverse side street effects will be. Third, the logic of TSP should be able to resolve the conflict between general traffic users and public transportation users and find an optimal balance.

The proposed logic is based on the idea that the bus could cooperate with the traffic signal to perform TSP. In contrast to current TSP logic where a bus approaching an intersection sends a priority request and the traffic controller tries to accommodate it without additional interactions, the proposed logic would include cooperation between the bus and traffic signal controller. The cooperation would require a bus to travel at a reasonable speed which is recommended based on remaining/expected queue, road geometry and normal signal timing plan. The proposed TSP logic would implement the green time reallocation, in other words, instead of adding additional green time to the original timing plan the proposed TSP logic splits the original green time and moves part of it to when green time is mostly needed by a transit bus. Furthermore, in addition to schedule adherence, the logic would account for delay per person as a conditional criterion to grant the TSP green time. The delay per person measurement indicates the trade-off between travel time saved by bus and travel time wasted by side streets. Therefore, the logic is expected to have more control on adverse side effects than basic "green extension" and "red truncation." Connected Vehicle (CV) technology will provide two-way communications between the bus and the traffic signal controller, including accurate bus location detection and prediction, and the number of passengers. Figure1 shows the architecture of the proposed TSP with Connected Vehicle technology for the One Bus Scenario with a nearside bus stop. This TSP logic takes advantage of the cooperation between transit buses and traffic signal to maximize flexibility and performance of TSP. It also utilizes vehicle level information to control adverse effect on side streets. The logic is composed of three major components:

1. Arrival time prediction component, in which two time ranges are predicted: bus arrival time ranges at the bus stop as well as at the subject intersection.

2. TSP timing plan and bus speed calculation component, in which, given the arrival time ranges, the algorithm generates a timing plan that will have minimum impact on general traffic users and calculates corresponding recommended bus speed.

3. Logic assessment and implementation component, in which the TSP timing plan will be compared against the normal signal time (winner overwrites the other) and the recommended bus speed will be transmitted to the coming bus. 


\section{Arrival Time Prediction Component}

One of the great advantages of integrating TSP into a CV system is having more information with better quality. Most importantly, the passenger counts on buses and potential passenger counts at the bus stops can be obtained. Utilizing this information, dwell time at the bus stops can be predicted more accurately.

Another feature of CVs which this study takes advantage of is the two-way communications between road side equipment (RSE) and traffic users which in this case is the bus. A CV-equipped bus communicates with traffic signal controller and is capable of receiving speed instructions. It is assumed that the desired speed of an approaching bus could vary between $10 \%$ above and $20 \%$ below the speed limit. Therefore, the prediction result generated from this component is not a fixed number; instead, it is a range of time. The range of arrival time is given so that the bus can adjust its travel at various speeds to cooperate with TSP strategy.

With the proposed logic, the arrival time is calculated using a simple equation derived from vehicle physics. The queue in front of bus will be cleared before bus arrives at the stop bar. Thus, the movement of the bus with a nearside bus stop will be:

$$
T_{\text {BusArrival }}=T_{c}+T_{d e c}+T_{a c c}+T_{d w e l l}
$$

Where:

$\mathrm{T}_{\mathrm{c}}$ : Travel time at near constant speed.

$\mathrm{T}_{\text {dec }}$ : Travel time that bus spends decelerating.

$\mathrm{T}_{\mathrm{acc}}$ : Travel time that bus spends accelerating.

$\mathrm{T}_{\mathrm{dwell}}$ : Time bus stopped at the bus stop (e.g., 30s).

It is noted that a far-side bus stop does not include bus' deceleration and acceleration before the intersection. For the arrival time prediction component, upper and lower bounds of arrival time at the bus stop and at the intersection are calculated to generate an expected range of arrival time.

\section{TSP Timing Plan and Bus Speed Calculation Component}

The TSP timing plan is calculated based on the goal that TSP green is inserted exactly where it is needed for the duration it is needed. The cycle length will be the same even when the TSP green is inserted, because the TSP green time is spliced from the original green time of the direction of the bus. So strictly speaking, the extra TSP green time is "moved" rather than "inserted" or "added." TSP green time is designed such that the bus will catch up with the end of the queue right at the stop bar of the intersection. The advantage of this approach is twofold:

1. Queue is cleared before the bus arrives at the intersection

2. The inserted green time taken from the certain direction is $100 \%$ used in clearing traffic for that direction. Therefore, theoretically speaking, not a single second is wasted during the TSP.

The calculation of the real-time queue length estimation presented below is based on the model developed by Liu [20] which is an extension of shock wave theory. 
The range of predicted bus arrival time was passed on from the last step for TSP timing plan calculation. Therefore, the computation finds a range of TSP green start time and end time. While there will be numerous TSP timing plans depending on when the bus arrives, the following rules were used in calculating TSP timing plan:

1. A TSP green time is preferred to start at the end of phase than to cut into the middle of a phase (for better safety and drivers' expectation)

2. If a TSP green has to start in the middle of a phase, it is preferred that the bus travels at its normal speed

3. Minimum green time is required for both the TSP green time and the original timing plan.

Based on these rules, the algorithm finds optimal TSP start and end times from the time range that TSP can possibly start and end. A mathematical model is run every time a TSP request is received, it find the value of choice variables $G_{1 \text { before }}, G_{2 b e f o r e}, G_{3 b e f o r e}$, and $T_{\text {TSPend }}$ to minimize total person delay at the intersection for three signal cycles. It is noted that three cycles were used to be long enough to capture residual effects caused by TSP and be short enough not to include another TSP, given three cycles of 160-second cycle is about the minimum headway between buses. The objective function estimating total person delay can be expressed as follows:

The choice variables include: $G_{1 \text { before}}, G_{2 \text { before }}, G_{3 \text { before, }}$ and $T_{\text {TSPend }}$.

$$
\operatorname{Minimize}\left(\sum_{\text {cycle }=1}^{\text {cycle }=3} D_{i} * O c c_{i}+\sum_{c y c l e=1}^{\text {cycle }=3} D_{b u s} * O c c_{b u s}\right)
$$

Which subject to:

$$
\begin{aligned}
& \sum_{j}\left(G_{\text {jbefore }}+G_{\text {jafter }}\right)+G_{T S P}+G_{\text {remain }}=\text { cyclelength }=\text { constant } \\
& T_{T S P e n d}-T_{T S P S}=G_{T S P} \\
& T_{\text {BAlow }} \leq T_{\text {TSPend }} \leq T_{\text {BAup }} \\
& T_{\text {TSPS }}=\sum_{j} G_{\text {jbefore }} \\
& G_{\text {jbefore }}+G_{\text {jafter }}=\text { Constant } \\
& G_{\text {jbefore }} \geq G_{\min } \text { or } G_{\text {jbefore }}=0 \\
& G_{\text {jafter }} \geq G_{\min } \text { or } G_{\text {jafter }}=0 \\
& G_{T S P} \geq G_{\min } \text { or } G_{T S P}=0 \\
& G_{\text {remain }} \geq G_{\text {min }} \text { or } G_{\text {remain }}=0
\end{aligned}
$$

Where: 
$\mathrm{D}_{\mathrm{i}}$ : Delay of vehicle $\mathrm{i}$

$\mathrm{D}_{\text {bus }}$ : Delay of bus

Occ $_{\mathrm{i}}$ : Occupancy on vehicle $\mathrm{i}$

Occ $_{\text {bus: }}$ : Occupancy on the bus

$\mathrm{G}_{\mathrm{jbefore}}$ : Green time for phase $\mathrm{j}(1,2$, or 3$)$ before TSP green

$\mathrm{G}_{\mathrm{jafter}}$ : Green time for phase $\mathrm{j}(1,2$, or 3$)$ after TSP green

$\mathrm{G}_{\mathrm{TSP}}$ : TSP green time

$\mathrm{G}_{\text {remain }}$ : Remaining green time for lane group with bus after taking out the TSP green

$\mathrm{G}_{\text {min }}$ : minimum green time requirement

$\mathrm{T}_{\text {TSPend }}$ : End time of TSP green

$\mathrm{T}_{\text {TSPs }}$ : Start time of TSP green

$\mathrm{T}_{\text {BAlow }}$ : Lower bound of bus arrival time range

$\mathrm{T}_{\mathrm{BAup}}$ : Upper bound of bus arrival time range

Once the timing plan is generated, the recommended bus speed could then be computed so that bus would travel through the intersection right after the queue in front is cleared and before TSP green phase ends.

Then, for the scenario with a nearside bus stop, there will be an extra step to update timing plan in case of extraordinary high volume. Since a bus stop is located on a bus' way to the intersection, it is possible that the queue in front could block the bus from loading and unloading. Then the previously predicted arrival time would not be accurate any more. In that case, the bus would miss the inserted TSP green. This situation cannot be anticipated until the speed of bus is found. Therefore, after the recommended speed of bus is computed, the logic is designed to double check if queue spillback happens with the initial TSP timing plan. In case queue spillback does happen, the TSP timing plan would be adjusted so that bus can get to the stop without impedance. However, the bus will have to wait for the normal green time to traverse through the intersection after it finishes loading and unloading.

\section{Logic Assessment and Implementation Component}

After a TSP timing plan is determined, the algorithm would compare the "with TSP" scenario against the "normal timing" scenario. Since the number of passengers on board is likely to be known under the CV environment, person delay performance measure is to be used. The person delay will be calculated for three consecutive signal cycles starting from the TSP implemented cycle. In this study, a TSP timing plan would be only implemented when its corresponding person delay is less than the "no TSP" scenario.

During implementation, two major steps are conducted. First, an instruction is given to a bus about the desired recommended speed. Second, a buffer green time is possibly given to a bus in case a bus is not expected to make it to the intersection. The TSP green time would be extended up to 5 seconds to accommodate the random delay.

\section{EVALUATIONS}

Both analytical tests and simulation evaluations have been performed for the proposed TSP as well as conventional TSP and no TSP cases. The test network is a calibrated VISSIM model of the intersection at Emmet St. and Barracks Rd. in Charlottesville, VA, as shown in Figure 2. Vehicle volumes and turning movements are actual morning peak-hour data collected from the 
site. In order to verify that the findings from the experiment are consistent with various congestion levels, a sensitivity analysis is also conducted. Four scenarios are tested: $\mathrm{v} / \mathrm{c}=0.5$, $\mathrm{v} / \mathrm{c}=0.7, \mathrm{v} / \mathrm{c}=0.9$ and $\mathrm{v} / \mathrm{c}=1.0$. Although there are currently no buses passing through the intersection, the research assumes a bus is traveling NB on Emmet St. with a mid-block bus stop located 750 feet upstream of the intersection. The speed limit on Emmet St. is $40 \mathrm{mph}$, therefore, buses are allowed to travel within the speed range between $30 \mathrm{mph}$ and $45 \mathrm{mph}$. The TSP logic is activated when buses pass 0.5 mile upstream of the intersection.

Both analytical tests and simulation evaluations compared three scenarios: without TSP (NTSP), conventional TSP (CTSP) and TSP with Connected Vehicle (TSPCV). The conventional TSP logic compared here is TSP with AVL and an on board passenger counting system. In other words, CTSP is the state-of-the-art TSP plus a more accurate bus arrival time forecast module. The difference between CTSP and TSPCV is that the logic CTSP utilizes is a simple one (green extension only) with no cooperative interactions between the bus and the traffic signal controller. The CTSP will grant 10-second extra green time to buses which arrive within 10 seconds of the end of normal green time. In case the bus could not make through the intersection within that 10 seconds, CTSP will add to the previous 10 seconds with up to 5 seconds to accommodate the late arrival. The logic follows the real implementation in the Northern Virginia [21].

\section{Analytical Test}

The cycle length at the intersection is 160 seconds. Assuming a TSP can be activated at any given second, there are 160 possible situations. Travel time for bus and for all traffic users are calculated for these 160 situations. FIGURE 3 shows bus travel times associated with various buses activating TSP over the cycle length at the intersection. The blue bars are bus travel time without TSP and red bars are travel time with TSPCV. It is observed that two scenarios overlap with each other for some portion of the chart. This is when a bus would travel through the intersection within the original green time without any impedance. Therefore, it is reasonable to see maximum time saving occurs right after the end of original green time and the savings decrease with time.

Another interesting observation is that bus travel time with TSPCV fluctuates with time. This is due to the rule specifying TSP is preferred to start from the end of phase than to cut into the middle of a phase or the minimum green time requirement. In these cases, the bus would have to travel faster or slower than at its normal speed. As a result, travel time varies, but still, transit buses are not stopped at the intersection.

While the conventional TSP is not shown in Figure 3 to keep the figure clean and more comprehensible, the pattern can be easily anticipated. Since the logic of conventional TSP is to extend green for extra a few seconds to let buses arriving right after the original green pass the intersection, the travel times of buses that arrive right after the dent (between 75 seconds to 85 seconds in FIGURE 3) would be as low as that of TSPCV condition (red bars). Hence, it can be expected that the travel time pattern of CTSP would be very similar to that of without TSP scenario with couple of the highest blue columns behind the dent drop to the red columns' height. The number of lowered bars would depend on how many seconds the TSP is allowed to extend the green time. But no matter how many seconds CTSP is extended, the delay time saving of conventional TSP would be less than that of TSPCV. 
TABLE 2 shows a comparison of analytical delay among three scenarios: without TSP (NTSP), conventional TSP (CTSP) and TSP with Connected Vehicle (TSPCV). The person delay is the average delay of all traffic users over three cycle lengths and then averaged over all 160 situations. The bus delay is the average bus waiting time of all 160 situations. The field collected volume is at $\mathrm{v} / \mathrm{c}=0.9$ level. The results of a sensitivity analysis of other $\mathrm{v} / \mathrm{c}$ ratios are presented and will be discussed in detail in the following section. In summary, during the morning peak, the proposed TSP logic (TSPCV) would reduce bus delay by $89.7 \%$ and reduce total delay by $5.6 \%$. In the same conditions, the conventional TSP would only reduce bus delay $12.6 \%$ and not reduce total delay. Intuitively, one may expect TSP to cause extra delay to the overall traffic, but this may not always be true. Because of the TSP green time, vehicles on the major street are being released on a higher frequency. As a result, the delay polygon would be smoothed out and never reach the old maximum. Thus, total delay would actually decrease, given the volume is not over saturated. In addition, considering the higher passenger density on bus, the time saving from bus riders will likely reduce the overall delay.

\section{Simulation-based Evaluation in VISSIM}

While the analytical test results show significant benefits under the proposed TSP logic, it does not consider any variability due to vehicle interactions and inter-arrival times. A microscopic traffic simulator can assess the performance of the proposed TSP under more plausible conditions. The microscopic simulation software package VISSIM [22] is used to evaluate the proposed TSP logic under a Connected Vehicle environment. A COM interface is used to assess information that would be available within a Connected Vehicle environment. The evaluation is performed under the assumption that only transit buses are connected to traffic signal controller and other traffic users do not have $\mathrm{CV}$ equipment. In other words, $0 \% \mathrm{CV}$ market penetration except for buses. The end of queue is estimated based on incoming vehicles and outgoing vehicles at the intersection. Detailed algorithm can be found in the model developed by Liu [20] which is an extension of shock wave theory. Therefore, the data extracted via COM interface [23] would only be speed and position of bus, number of passengers on board, number of potential passenger at the bus stop, number of vehicles passing the intersection and volume from all four approaches. Also, the COM interface is used to change signal timing plan during the simulation. All programs are coded in Microsoft EXCEL VBA.

As noted, the test network is a calibrated model of the intersection at Emmet St. and Barracks Rd. in Charlottesville, VA. Vehicle volumes and turning movements are actual morning peak-hour data collected from the site. Bus dwell time at the stop is 30 seconds average with a standard deviation of 2 seconds. A transit bus is designed to arrive every 494 seconds. Given the cycle length is 160 seconds at the intersection; the interval of bus arrival is about 3 cycles. During the evaluation, the team discovered that the simulation evaluation of CTSP could be easily biased. In order to include as many arrival scenarios as possible, the headway between buses should not be an exact multiple of signal cycles. Otherwise, all buses would arrive at the same specific time relative to the signal cycle. Therefore, there should be a small offset added to the arrival interval to include various arrival scenarios. But, if this offset is less than TSP green extension time, and because the TSP extension causes the original green phase to shift the same direction as the bus arrival time, as long as one bus gets TSP, all following buses would be included in the green phase. Therefore, this research purposefully designed the offset to be 14 seconds so that a sequence of buses will arrive at various times relative to signal cycles without causing the domino effect; hence the simulation result would be less biased. 
The simulation based evaluations also compared three scenarios: without TSP (NTSP), with conventional TSP (CTSP) and TSP with Connected Vehicle (TSPCV). Each scenario ran at least five times with different random seeds. It is ensured that the results show statistical significance with a 95\% confidence level and 5\% tolerance error. The sample size computation formula documented in the Traffic and Highway Engineering textbook written by Garber and Hoel [24] was used. Five runs are sufficient in this case.

The simulation based results shown in Table 3 support the results from analytical tests. Delay per person is approximately on the same level for all three scenarios and so is the bus delay. Although the delay of all traffic users for conventional TSP is slightly lower than the analytical result, statistical tests show that the three scenarios do not have statistically significant difference on delay of all traffic users. Therefore, the simulation results are consistent with those of the analytical model in terms of person delay. For bus delay, CTSP seems to perform better than expected while TSPCV performs a bit worse than expected. Closer investigation shows that this phenomenon is caused by the bias of CTSP simulation evaluation. Although the bus arrival time has been designed to shift 14 seconds every time a new bus comes to minimize the bias, this bias cannot be eliminated. Because the CTSP green shifts the green cycle 10-15 seconds the same direction as bus arrival time, their synchronization increases with the number of buses helped by CTSP. But this would not be the case in real world as bus arrival time and green cycles do not shift in the same direction. As for TSPCV, the lower than expected performance is caused by a couple of buses that do not make through the intersection within TSP green time due to randomness of traffic and dwell time at the bus station.

The TSP with Connected Vehicle (TSPCV) shows better performance over the conventional TSP in terms of bus travel time savings. It reduces the bus delay by $50.0 \%$ compared to conventional TSP and $60.1 \%$ compared to NTSP condition.

This research also compared the standard deviation of bus travel times as a measurement to represent the reliability of bus service. Table 3 demonstrates that TSPCV improves reliability of bus service while CTSP is actually less reliable than no-TSP condition. However, the statistical tests show that the reliability difference between NTSP and CTSP is not statistically significant.

\section{Sensitivity Analysis on Congestion Levels}

In order to verify that the findings from the experiment are consistent with various congestion levels, a sensitivity analysis is conducted. Since the field collected volume data is at v/c ratio 0.9, three other scenarios are tested: $\mathrm{v} / \mathrm{c}=0.5, \mathrm{v} / \mathrm{c}=0.7$ and $\mathrm{v} / \mathrm{c}=1.0$. The results have been presented in TABLE 2 and Table 3.

Both analytical and simulation evaluation results show similar trends with respect to how TSPCV performs under various congestion levels. In terms of bus delay at the intersection, TSPCV always reduces bus delay compared to conventional TSP and NTSP conditions. It should be considered that the analytical evaluation is to obtain upper ceiling benefits, while the simulation evaluation is to assess expected performance under real world implementation.

When the congestion level is low, TSPCV would help reduce bus delays up to about $90 \%$ compared to NTSP under VISSIM simulations. As the congestion level rises, the benefit of TSPCV decreases, while no extra delay is caused. This is because the algorithm is designed to be 
conditional on person delay. When the volume becomes closer to the capacity, less portion of the green time will be granted to TSPCV to prevent TSP from causing extra delay on other travelers. As a result, the benefit would drop correspondingly, while adverse effects on side streets would still be kept under a certain level. It is interesting to see that even when $\mathrm{v} / \mathrm{c}$ ratio equals to 0.9 , the benefit of TSPCV is still significant and drops dramatically when $\mathrm{v} / \mathrm{c}$ becomes 1.0. However, even when $\mathrm{v} / \mathrm{c}=1.0, \mathrm{TSPCV}$ is still superior to conventional TSP.

As noted, per person delay at the intersection is a measurement that reflects adverse effects caused by TSP. When examining the results, both CTSP and TSPCV did not cause additional person delay at various $\mathrm{v} / \mathrm{c}$ ratios. For low $\mathrm{v} / \mathrm{c}$ ratio scenarios $(\mathrm{v} / \mathrm{c}<0.9)$, TSPCV person delays are lower than those of CTSP. As the congestion level increases, the difference of person delay between TSPCV and CTSP decreases and eventually becomes statistically insignificant.

\section{CONCLUSIONS}

To address challenges identified in the current TSP strategies, a next generation TSP logic based on Connected Vehicle technology is proposed. This new TSP takes advantage of the two-way communications and additional and more accurate information provided by Connected Vehicle technology. Based on the simulation results, it can be concluded that the proposed TSP provides buses with more accuracy and better effectiveness. Furthermore, it accommodates a higher percentage of transit buses than conventional TSP. Its performance is compared against conventional TSP (CTSP) and no-TSP (NTSP) conditions under various congestion levels. The results show that the TSPCV would greatly reduce bus delay at signalized intersection without causing negative effects on side streets.

The performance of TSPCV was evaluated under various congestion conditions, including near capacity volume condition. The results show that, at all congestion levels, TSPCV outperforms both CTSP and NTSP conditions. Although the benefit would be small during peak hour, little adverse effects on side streets are expected. Hence, it will no longer be a must for local agencies and DOTs to perform a study of LOS and/or V/C ratio for potential TSP intersections before installation.

The last important point to make is that TSPCV is one of the few ITS applications that would generate benefits even at early stages of Connected Vehicle technology deployment. The quantitative evaluation performed in this research is based on the assumption that only buses are equipped with Connected Vehicle technology (i.e., DSRC) devices. This feature also brings out another character of TSPCV that its deployment cost is moderate compared to other Connected Vehicle applications: the application only needs equipment upgrades on buses and traffic signal controllers. Thus, TSPCV could be a good starting point to promote Connected Vehicle technology. 


\section{ACKNOWLEDGEMENTS}

This research project was in part supported by the Mid-Atlantic University Transportation Center, Connected Vehicle/Infrastructure University Transportation Center and the Virginia Center for Transportation Innovation and Research. The authors are grateful to Mr. Peter Ohlms at VCTIR and Mr. Amit Sidhaye at Arlington County for their help for understanding Virginia's transit signal priority status.

The paper has been presented at the $93^{\text {rd }}$ Annual Meeting of the Transportation Research Board, January 2014, Washington, D.C., and accepted for publication in the 2014 series of the Transportation Research Record: Journal of the Transportation Research Board (forthcoming). The material from your paper is reproduced with permission of the Transportation Research Board. 


\section{REFERENCES}

[1] Hill, C. J., and J. K. Garrett. AASHTO Connected Vehicle Infrastructure Deployment Analysis. Publication FHWA-JPO-11-090. FHWA, U.S. Department of Transportation, 2011.

[2] Intelligent Transportation Systems Joint Program Office. Updated Descriptions on USDOT High-Priority Dynamic Mobility Applications. Research and Innovative Technology Administration, U.S. Department of Transportation. http://www.its.dot.gov/press/2011/mobility app.htm. Accessed April 182013.

[3] Volpe J. A.. Vehicle-Infrastructure Integration (VII) Initiative Benefit-Cost Analysis Version 2.3 (Draft), National Transportation Systems Center, Federal Highway Administration, 2008.

[4] Muthuswamy, S., W. R. McShane, and J. R. Daniel. Evaluation of Transit Signal Priority and Optimal Signal Timing Plans on Transit and Traffic Operations. November 15, 2006, revised and submitted for inclusion in the CD-ROM and possible publication to: Transportation Research Board86thAnnual Meeting, January 2007, Washington, D.C.

[5] Liao, C., and G. A. Davis. Simulation Study of a Bus Signal Priority Strategy Based on GPS, AVL and Wireless Communications. Revised and submitted for inclusion in the CD-ROM and possible publication to: Transportation Research Board 86th Annual Meeting, Washington, D.C., January 2007.

[6] Al-Sahili, K. A., and W. C. Taylor. Evaluation of bus priority signal strategies in Ann Arbor, Michigan. In Transportation Research Record: Journal of the Transportation Research Board, No. 1554, Transportation Research Board of the National Academies, Washington, D.C., 1996, pp. 74-79.

[7] Shalaby, A., J. Lee, G. S. Hung and M. D. Bowie, Development, Evaluation, and Selection of Advanced Transit Signal Priority Concept Directions, Journal of Public Transportation, Vol. 9, No. 5, 2006.

[8] Gifford, J., D. Pelletiere, and J. Collura, Stakeholder Requirements for Traffic Signal Preemption and Priority in Washington, D.C., Region. In Transportation Research Record: Journal of the Transportation Research Board, No. 1748, Transportation Research Board of the National Academies, Washington, D.C., 2003, pp. 1-7.

[9] Lee, J., A. Shalaby, J. Greenough, M. Bowie and S. Hung. Advanced Transit Signal Priority Control with Online Micro-simulation-Based Transit Prediction Model. In Transportation Research Record: Journal of the Transportation Research Board, No. 1925, Transportation Research Board of the National Academies, Washington, D.C., 2005, pp. 185-194.

[10] Balke, K. N., C. L. Dudek and T. Urbanik II. Development and Evaluation of Intelligent Bus Priority Concept. In Transportation Research Record: Journal of the Transportation Research Board, No. 1727, Transportation Research Board of the National Academies, Washington, D.C., 2000, pp. 12-19.

[11]Ekeila, W., T. Sayed and M. El Esawey, Development of Dynamic Transit Signal Priority Strategy, In Transportation Research Record: Journal of the Transportation Research Board, No. 2111, Transportation Research Board of the National Academies, Washington, D.C., 2009, pp. 1-9.

[12] Chang, G., M. Vasudevan and C. Su. Modeling and evaluation of adaptive bus-preemption control with and without automatic vehicle location systems. In Transportation Research Part A: Policy and Practice, No. 30 A, Transportation Research Board of the National Academies, Washington, D.C., 1996, pp. 251-268. 
[13] Skabardonis, A.. Control Strategies for Transit Priority. In Transportation Research Record: Journal of the Transportation Research Board, No. 1727, Transportation Research Board of the National Academies, Washington, D.C., 1998, pp. 20-26.

[14] Transit Cooperative Research Program. Traveler Response to Transportation System Changes: Interim Handbook. TCRP Project B-12. TRB, National Resource Council, Washington, D.C., Mar. 2000. http://onlinepubs.trb.org/onlinepubs/tcrp/tcrp webdoc 12.pdf. Accessed July 15, 2012.

[15]Chang, J., J. Collura, F. Dion and H. Rakha. Evaluation of Service Reliability Impacts of Traffic Signal Priority Strategies for Bus Transit. In Transportation Research Record: Journal of the Transportation Research Board, No. 1841, Transportation Research Board of the National Academies, Washington, D.C., 2003, pp. 23-31.

[16] Eleni, C. Traffic Signal Optimization with Transit Priority: A Person-based Approach. University of California, Berkeley. http://search.proquest.com//docview/1081483605, accessed July 20, 2013.

[17] Mirchandani, P, A. Knyazyan, L. Head, W. Wu. An Approach Towards the Integration of Bus Priority, Traffic Adaptive Signal Control, and Bus Information. Scheduling Systems Lecture Notes in Economics and Mathematical Systems Volume 505, 2001, pp 319-334. http://link.springer.com/chapter/10.1007/978-3-642-56423-9_18, accessed July 12, 2013.

[18] Multi-Modal Intelligent Traffic Signal System, University of Arizona, http://cts.virginia.edu/PFS_MMITSS02_Task1_PMP.pdf, accessed Jan 13, 2013.

[19] Rakha, H. and K. Ahn, Transit signal priority project - phase II- Simulation study results. VTRC 06-CR. Contract Research Sponsored by Virginia Transportation Research Council, Jan, 2006.

[20]H. X. Liu, X. Wu, W. Ma, and H. Hu, Real-time queue length estimation for congested signalize intersections, Transportation research Part C 17 (2009) 412-427.

[21] Rakha, H. and K. Ahn, Transit signal priority project - phase II- Field Evaluation results. VTRC 06-CR. Contract Research Sponsored by Virginia Transportation Research Council, Jan, 2006.

[22] PTV, "VISSIM 5.10 User Manual," Jul. 2008.

[23]PTV, "VISSIM 5.10-03 COM Interface Manual," Sep. 2008.

[24] Garber, N. J. and L. A. Hoel, Traffic and Highway Engineering, Fourth Edition, Nelson Education Ltd., Canada, 2008, Page 103. 


\section{LIST OF TABLES}

TABLE 1 Summary of TSP Benefits/Disbenefits .................................................... 28

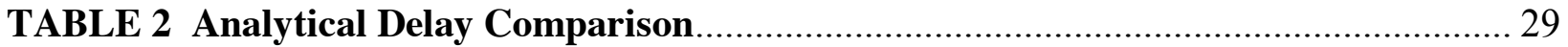

\section{LIST OF FIGURES}

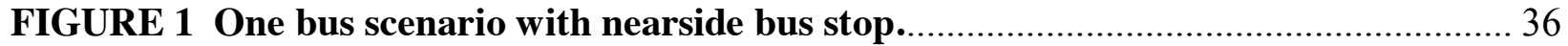

FIGURE 2 Study site-Emmet St and Barracks Rd intersection, Charlottesville............. 30

FIGURE 3 Bus travel time (without TSP compared to TSPCV). ................................. 31 
TABLE 1 Summary of TSP Benefits/Disbenefits

\begin{tabular}{|c|c|c|c|c|c|}
\hline Location & TSP Type & \multicolumn{2}{|c|}{ Measurements } & Result & Reference \\
\hline \multirow{2}{*}{ Newark, NJ } & \multirow{2}{*}{ Conventional } & \multirow{2}{*}{$\begin{array}{l}\text { Travel } \\
\text { Time }\end{array}$} & Bus & $\begin{array}{l}10 \%-20 \% \\
\text { decrease }\end{array}$ & \multirow{2}{*}[4]{} \\
\hline & & & $\begin{array}{l}\text { Auto (Main } \\
\text { St) }\end{array}$ & $5 \%-10 \%$ decrease & \\
\hline \multirow{2}{*}{ Minneapolis } & \multirow{2}{*}{$\begin{array}{l}\text { TSP with } \\
\text { AVL }\end{array}$} & \multirow{2}{*}{$\begin{array}{c}\text { Bus Travel } \\
\text { Time } \\
\end{array}$} & AM Peak & $12-15 \%$ decrease & \multirow{2}{*}[5]{} \\
\hline & & & PM Peak & 4- $11 \%$ decrease & \\
\hline \multirow{2}{*}{$\begin{array}{l}\text { Ann Arbor, } \\
\text { Michigan }\end{array}$} & \multirow{2}{*}{ Conventional } & \multirow{2}{*}{ Delay } & Bus & little benefit & \multirow{2}{*}[6]{} \\
\hline & & & Auto & increase & \\
\hline \multirow{3}{*}{$\begin{array}{l}\text { Hypothetical } \\
\text { Intersection }\end{array}$} & \multirow{3}{*}{$\begin{array}{l}\text { TSP with } \\
\text { AVL }\end{array}$} & \multirow{2}{*}{$\begin{array}{l}\text { Average } \\
\text { Bus Delay }\end{array}$} & Non Peak & $24.81 \%$ decrease & \multirow{3}{*}{ [9] } \\
\hline & & & Peak & $-28.92 \%$ & \\
\hline & & \multicolumn{2}{|c|}{ Side Street Delay } & $\begin{array}{c}23.30 \%-55.23 \% \\
\text { increase }\end{array}$ & \\
\hline \multirow{2}{*}{$\begin{array}{l}\text { Hypothetical } \\
\text { network with } 3 \\
\text { intersections }\end{array}$} & \multirow[t]{2}{*}{$\begin{array}{l}\text { TSP with } \\
\text { AVL }\end{array}$} & \multirow[t]{2}{*}{$\begin{array}{l}\text { Stop } \\
\text { Delay }\end{array}$} & $\begin{array}{c}\text { Veh in } \\
\text { Bus's } \\
\text { Direction }\end{array}$ & $6 \%-10 \%$ decrease & \multirow[t]{2}{*}[10]{} \\
\hline & & & Cross Street & $2 \%-26 \%$ & \\
\hline \multirow{2}{*}{ Vancouver } & \multirow{2}{*}{$\begin{array}{l}\text { TSP with } \\
\text { AVL }\end{array}$} & \multirow{2}{*}{\multicolumn{2}{|c|}{$\begin{array}{l}\text { Bus Travel Time } \\
\text { Cross Street Delay }\end{array}$}} & $33 \%$ & \multirow{2}{*}[11]{} \\
\hline & & & & Not significant & \\
\hline $\begin{array}{l}\text { Hypothetical } \\
\text { Intersection }\end{array}$ & $\begin{array}{l}\text { Adaptive } \\
\text { TSP }\end{array}$ & \multicolumn{2}{|c|}{ Total Delay } & $\begin{array}{c}3.04 \%-71.03 \% \\
\text { decrease }\end{array}$ & {$[12]$} \\
\hline \multirow{4}{*}{ Arlington, Virginia } & \multirow{4}{*}{$\begin{array}{l}\text { TSP with } \\
\text { AVL }\end{array}$} & \multicolumn{2}{|c|}{ Reliability } & $3.20 \%$ & \\
\hline & & \multicolumn{2}{|c|}{ Bus Travel Time } & $-0.90 \%$ & \\
\hline & & Total & Per Veh & $1 \%$ & \\
\hline & & Delay & Per Person & $0.60 \%$ & \\
\hline \multirow[b]{2}{*}{ Portland, OR } & & Bus Tra & vel Time & $10 \%$ decrease & \\
\hline & Conventional & On-Time & erformance & $\begin{array}{c}8 \%-10 \% \\
\text { improvement }\end{array}$ & \\
\hline & & Priority & Bus Delay & $34 \%$ decrease & \\
\hline Seattle, WA & Conventional & Bus Inters & ction Stops & $24 \%$ decrease & [14] \\
\hline & & Bus Tra & vel Time & $8 \%$ decrease & \\
\hline Los Angeles, CA & Conventional & Bus Tra & vel Time & $8 \%-10 \%$ decrease & \\
\hline Rremerton WA & Conventional & Bus Tra & vel Time & $10 \%$ decrease & \\
\hline Bremerton, w A & Conventional & Stopped D & lay/Vehicle & Insignificant & \\
\hline Chicago, IL & Conventional & Bus Tro & vel Time & $\begin{array}{l}\text { 2-3-min decrease } \\
\text { from } 13-17 \mathrm{~min}\end{array}$ & \\
\hline Northern Virginia & $\begin{array}{l}\text { TSP with } \\
\text { AVL }\end{array}$ & Bus Tra & vel Time & $\begin{array}{c}\text { Insignificant } \\
\text { benefit }\end{array}$ & [19] \\
\hline
\end{tabular}


TABLE 2 Analytical Delay Comparison

\begin{tabular}{ccccccc}
\hline & $\begin{array}{c}\text { v/c } \\
\text { ratio }\end{array}$ & NTSP & CTSP & TSPCV & N/TSPCV & C/TSPCV \\
\hline Bus & 0.5 & 57.7 & 50.3 & 5.3 & $-90.8 \%$ & $-89.5 \%$ \\
Delay & 0.7 & 57.7 & 50.3 & 5.3 & $-90.8 \%$ & $-89.5 \%$ \\
(Sec) & 0.9 & 57.7 & 50.4 & 5.9 & $-89.7 \%$ & $-88.3 \%$ \\
& 1.0 & 57.7 & 51.1 & 27.1 & $-53.0 \%$ & $-46.8 \%$ \\
Delay per & 0.5 & 46.8 & 46.8 & 44.1 & $-5.6 \%$ & $-5.6 \%$ \\
person & 0.7 & 46.8 & 46.8 & 44.1 & $-5.6 \%$ & $-5.6 \%$ \\
(Sec) & 0.9 & 49.5 & 49.5 & 46.7 & $-5.6 \%$ & $-5.6 \%$ \\
& 1.0 & 51.1 & 51.1 & 48.3 & $-5.6 \%$ & $-5.5 \%$ \\
\hline
\end{tabular}

TABLE 3 Simulation Delay Comparison

\begin{tabular}{ccccccc}
\hline MOE & v/c ratio & NTSP & CTSP & TSPCV & N/TSPCV & C/TSPCV \\
\hline Bus Delay & 0.5 & 39.1 & 27.5 & 5.6 & $-85.7 \%$ & $-79.7 \%$ \\
(Sec) & 0.7 & 40.6 & 29.2 & 4.8 & $-88.3 \%$ & $-83.7 \%$ \\
& 0.9 & 45.4 & 36.3 & 18.1 & $-60.1 \%$ & $-50.0 \%$ \\
& 1.0 & 48.7 & 34.0 & 30.9 & $-36.5 \%$ & $-9.2 \%$ \\
Delay per & 0.5 & 36.1 & 33.0 & 30.9 & $-14.3 \%$ & $-6.2 \%$ \\
person & 0.7 & 37.6 & 34.7 & 33.2 & $-11.6 \%$ & $-4.3 \%$ \\
(Sec) & 0.9 & 42.8 & 39.2 & 40.3 & $-5.9 \%$ & $2.6 \%$ \\
$\begin{array}{c}\text { STDDEV } \\
\text { (sec) }\end{array}$ & 1.0 & 52.0 & 44.5 & 45.7 & $-12.0 \%$ & $2.8 \%$ \\
\hline & All & 31 & 32 & 26 & $-16.1 \%$ & $-18.8 \%$ \\
\hline
\end{tabular}




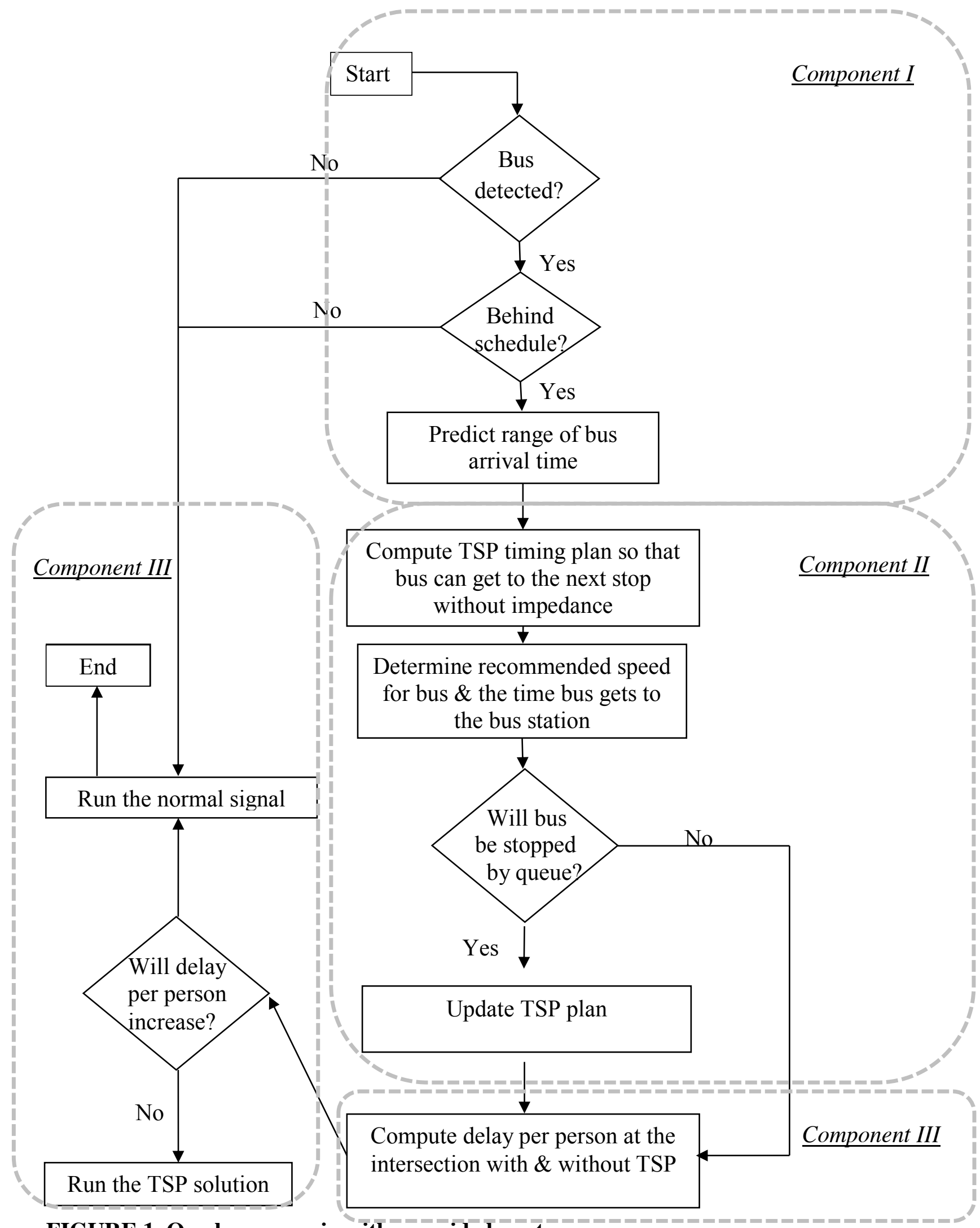

FIGURE 1 One bus scenario with nearside bus stop. 


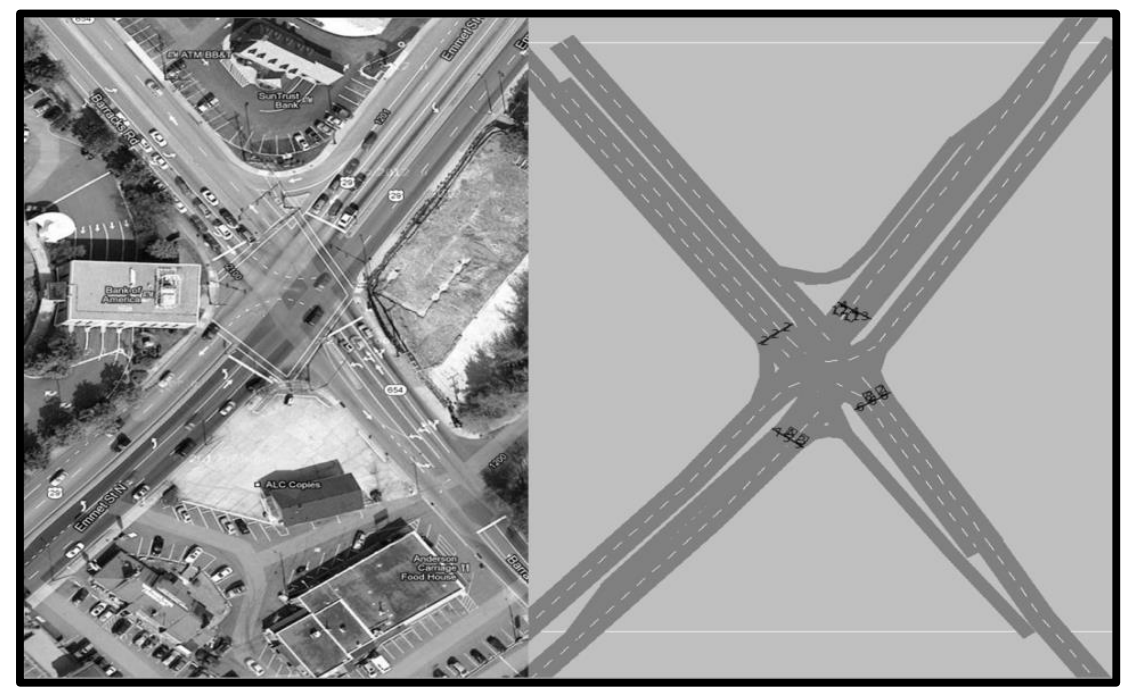

FIGURE 2 Study site-Emmet St and Barracks Rd intersection, Charlottesville.

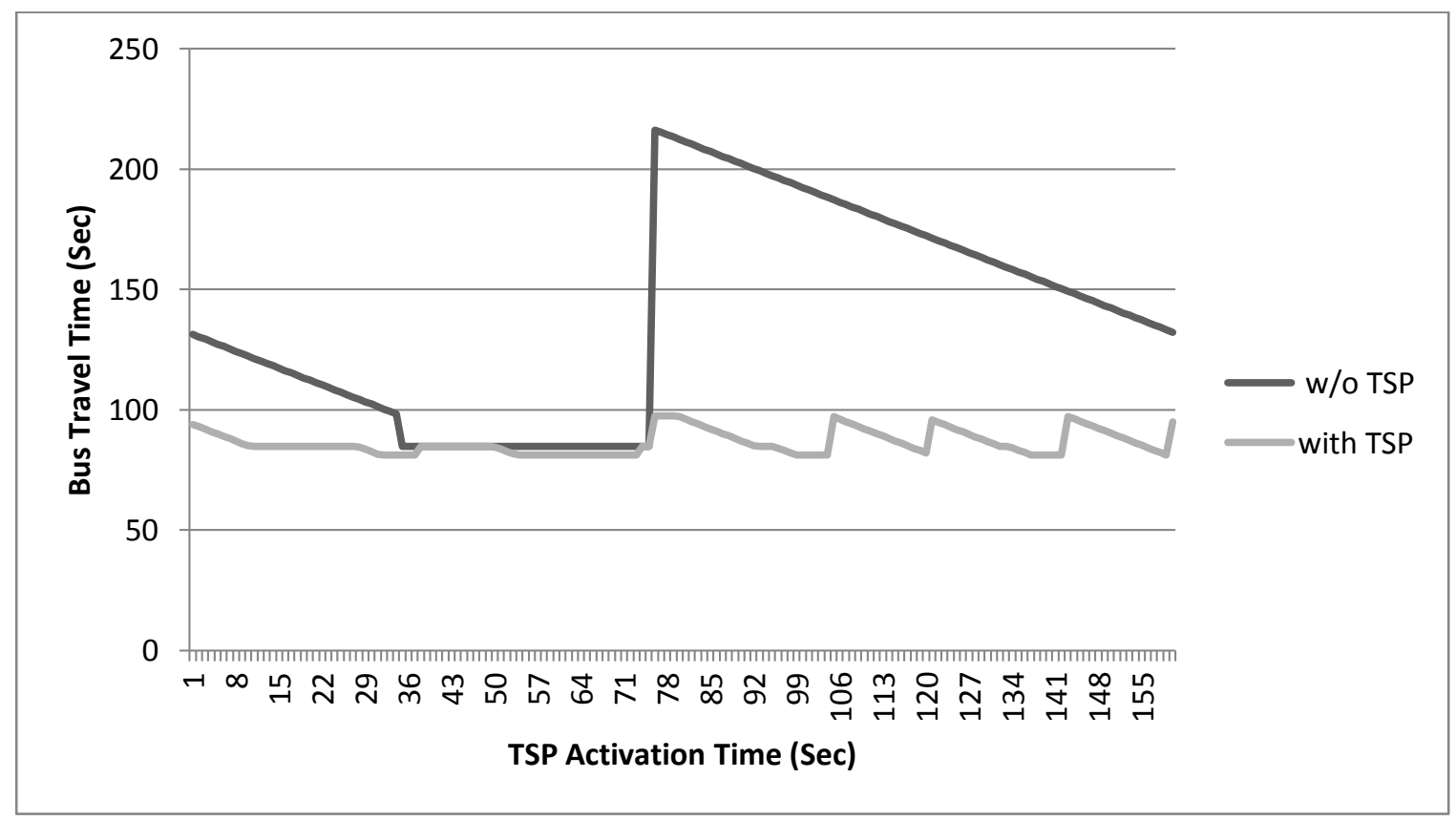

FIGURE 3 Bus travel time (without TSP compared to TSPCV). 


\title{
NEAR-SIDE BUS STOP WITH QUEUE JUMPER LANE UNDER CONNECTED VEHICLES
}

\author{
Jia $\mathrm{Hu}^{* 1)}$ \\ Ph.D. Candidate
}

Tel: 919-744-9842. Email: jh8dn@virginia.edu

\author{
Byungkyu "Brian" Park, Ph.D. 1) \\ Associate Professor
}

Tel: (434) 924-6347, Email: bpark@virginia.edu

\author{
Robert Robison ${ }^{1)}$ \\ Undergraduate Research Assistant
}

Tel: (434) 249-7601, Email: rar4dx@virginia.edu

Amit Sidhaye, $\mathbf{P E}^{2)}$

Project Manager

Tel: (703) 228-3730, Email: asidhaye@arlingtonva.us

2) Department of Civil and Environmental Engineering

University of Virginia

P.O. Box 400742

Charlottesville, VA 22904-4742

Fax: (434) 982-2951

\section{3) Department of Environmental Services, Division of Transportation Arlington County, VA 22201}

\begin{abstract}
Many bus stops in the urban area in the US are near-side. A major limitation of near-side bus stop is that buses lose time when they exit the near-side bus stop to make a sharp serpentine merging maneuver to continue through the intersection. A potential solution to this limitation is to add a queue jumper lane to near-side bus stop. This paper investigates multiple designs of queue jumper at the bus stop including one recently implemented in Arlington County, VA. Then an enhanced queue jumper lane at near-side bus stop (i.e., queue jumper bus stop) with Connected Vehicle (CV) technology is proposed and evaluated. A microscopic traffic simulation based evaluation reults indicated that the bus stop with CV saves the bus delay by about $30 \%$ and outperforms other alternatives by at least $15 \%$. Additionally, queue jump bus stop with CV shows no adverse effect on other traffic users.
\end{abstract}

Key words: Queue Jumper Lane; Near-side Bus Stop; Intelligent Transportation System; Connected Vehicle; Bus Priority; Delay 


\section{INTRODUCTION}

Bus stops are generally categorized into three kinds: near-side, far-side, and mid-block. They all have scenarios where they are more beneficial than the other stops. Although, each kind of bus stop has advantages and disadvantages, near-side is found to be the most commonly used in many highly populated areas such as Houston and Washington D.C. (1). As a matter of fact, the majority of D.C. bus stops are near-side and $80 \%$ of Houston's are near-side (2). Hence, the performance of a near-side bus stop greatly influences the overall performance of a city's transit system.

By definition, near-side bus stops are stops that are located immediately before an intersection. These stops are commonly utilized if there are a great number of pedestrians on the near-side and heavier traffic on the far-side of the intersection. Near-side stops may also be particularly worthwhile if the bus route requires a right-hand turn at the intersection. The main disadvantages to near-side bus stops as far as traffic flow is concerned are: 1, buses conflict with right-turning vehicles, it is very difficult for them to merge back in with traffic after dwelling; 2 , queues can potentially prevent buses from entering the bay in peak traffic hours. These downsides cause a great increase on bus delay. By solving these problems, the performance of a city's transit system would be considerably improved. Therefore, it is necessary to come up with a mechanism to help buses with near-side stops reach bus stops fast and make efficient merging maneuver.

One potential solution is to add Queue Jumper Lane (QJL) to the near-side stops. Queue jumper lane is a short lane that allows buses or other high priority vehicles to bypass the accumulated queues at intersections. It is usually accompanied by a traffic signal that allows all the transit buses in the queue jumper lane to leave before the vehicles in the other lanes. Essentially, if a bus arrives at the near-side stop while the traffic signal is red, it would receive a head-start on other vehicles at the intersection.

A new emerging system known as connected vehicles is able to provide real-time traffic data and thus create many possibilities. In a connected vehicles environment, diagnostic sensors are installed on every vehicle to collect data and data are being transmitted wirelessly between vehicles and nearby infrastructures. It would no longer have to rely on conventional data collection equipments, like loop detectors or video detections, and it collects much more information than the conventional ways. Measurements that are previously unknown are now available, which include but not limited to: vehicle speeds, positions, arrival rates, rates of acceleration and deceleration, queue lengths, stopped time and so on. By taking advantage of these extra information, more advance queue jumper lane mechanism can be developed.

Currently, no study has been conducted in the US regarding performance of near-side bus stop equipped with queue jumper lane, since queue jumper lane is usually considered as a supplement for transit signal priority (3) instead of a bus stop design. When it comes to bus stop design, most studies have focused on determining the optimum location of a bus stop (4-6). There is only one study tested the performance of bus stop with queue jumper lane and significant benefit was discovered when volume was above $250 \mathrm{vphpl}$. However, the bus stop investigated was a far-side bus stop and the queue jumper lane design was a simple infrasture without signal priority. 
Several analyses have evaluated the efficiency of Transit Signal Priority (TSP) with queue jumper lanes. In a VISSIM microscopic simulation comparing TSP with and without queue jumper lanes, the results showed that adding the queue jumper lane reduced bus delays by up to $50 \%(7,8)$. The other factors, such as intersection, minor, and major street delays were not significantly affected by the addition. In other words, providing buses with early green benefits more when they have their own queue jumper lane. The study also found that TSP with queue jumper lane was much more efficient with the bus stop located closer to the jumper lane. However, the study only addressed the conditions that queue jumper lane was placed downstream of a bus stop. Therefore, this design would not benefit buses that need to merge back into traffic from near-side bus stop. In addition, this previous study focused mainly on TSP logic. Queue jumper lane was merely one part of a large signal priority system. They did not evaluate the impact of queue jumper lane on near-side bus stop performance.

A research analyzing the addition of jumper lanes to already existing signal priorities noted that without jumper lanes TSP did not work well during peak hours, when it was needed the most (9). A major merit of queue jumper lanes would be their efficiency at peak hours, when normal TSP struggles. A possible disadvantage, however, was the possibility of the jumper lane being blocked off because the normal queue was so long. This problem can be resolved through moving the bus stop to the beginning of the lane, or extending the lane. Again, same as the previous research, they did not address the condition when a bus stop is located right next to the intersection.

Researchers at FIU tested queue jumper lanes containing multiple TSP strategies, traffic volumes, and dwell times (10). They found that queue jumper lanes without TSP were ineffective in reducing delays. Basically, even if there is a specific lane just for buses, but without phase insertion or early green, no benefit would be gained. However, the TSP logic proposed in that research was complicated. It is possible that a queue jumper lane might function well without sophisticated TSP logic. Therefore, it is beneficial to analyze how helpful implementing simple head-start phase insertion in combination with queue jumper lane is when dealing with current near-side bus stops.

The review of the research activities on the queue jumper lane implies the following findings. Firstly, there is no systematic investigation completed merely for near-side bus stop with queue jumper lane, nor is quantitative study performed to evaluate the recently implemented near-side bus stop with queue jumper lane. However, it is important to understand the nature of queue jumper bus stop facility and find out whether it has an adverse effect on other traffic users. Secondly, the current implemented QJL offers the possibility of improvement by introducing CV technology. By taking advantage of the resources provided by $\mathrm{CV}$, including two-way communications between the bus and the traffic signal controller, more efficient mechanism can be developed. Finally, the Arlington queue-jumper near-side bus stop has separated right turn lane and queue jumper lane. It is not always possible to obtain such geometry in the US, especially in the metropolitan area where space is constrained. Therefore, it is valuable to determine whether combining the only existing right turn lane with queue jumper lane is beneficial enough to warrant widespread implementation. 


\section{Goals and Objectives}

In summary, the objectives of this paper are three-fold.

1. Validate the existing facility in Arlington using a microscopic traffic simulation.

2. Propose an enhanced queue jumper bus stop using CV technology.

3. Test other feasible innovative alternatives of realizing queue jumper function on nearside bus stop.

\section{STUDY SITE}

Arlington County in Virginia, USA recently implemented a queue-jumper signal with a bus-only phase on southbound Glebe Road at Henderson. The location was problematic due to the need for a bus exiting the near-side bus stop to make a sharp serpentine merging maneuver to continue through the intersection. A dual right-turn lane was converted to a bus-only bus stop zone and, a right-turn lane to the left of the bus zone. Inductive loops in the bus-only zone detect stopped buses and a 7 -second bus-only phase would then be inserted before the normal southbound Glebe through movement. During the bus-only phase, a "NO RIGHT TURN" sign is illuminated and a green ball is shown that is louvered to be visible only from the bus zone. While the addition of this queue jumper lane function receives positive feedback from the bus drivers, the design is not supported by engineering and/or scientific study. One of the major concerns is whether this mechanism causes adverse effect to other traffic users. In other words, quantitative performance of this new facility is not available, nor is the impact of this design on the general traffic.

The intersection used in this study is located at the Glebe and Henderson intersection in Arlington County, VA. It is the only intersection in the United States that currently has a queue jumper lane setup on a near-side bus stop. It is a high volume intersection with $1500 \mathrm{vph}$ coming from southbound, which is about $84 \%$ of the capacity. The queue jumper lane is in the southbound direction, and an extra bus only lane is located to the right of the right-hand turn lane. The near-side bus stop is right before the traffic light, making it more convenient for pedestrians, but more difficult to determine when the early green (for queue jumper lane) should be given. While CV technology would help assigning early green more accurately and efficiently.

Figure 1 shows the geometry of the study site. The north and south bound directions have two lanes each. The southbound expands to four lanes before the intersection including a right-turn lane and a queue jumper lane. The northbound remains two before the traffic light. The east and west bound directions have less traffic, and therefore less green time every cycle. They both have two lanes approaching the intersection. The westbound expands to three lanes before the intersection including an exclusive right-turn lane and a left-turn lane. 


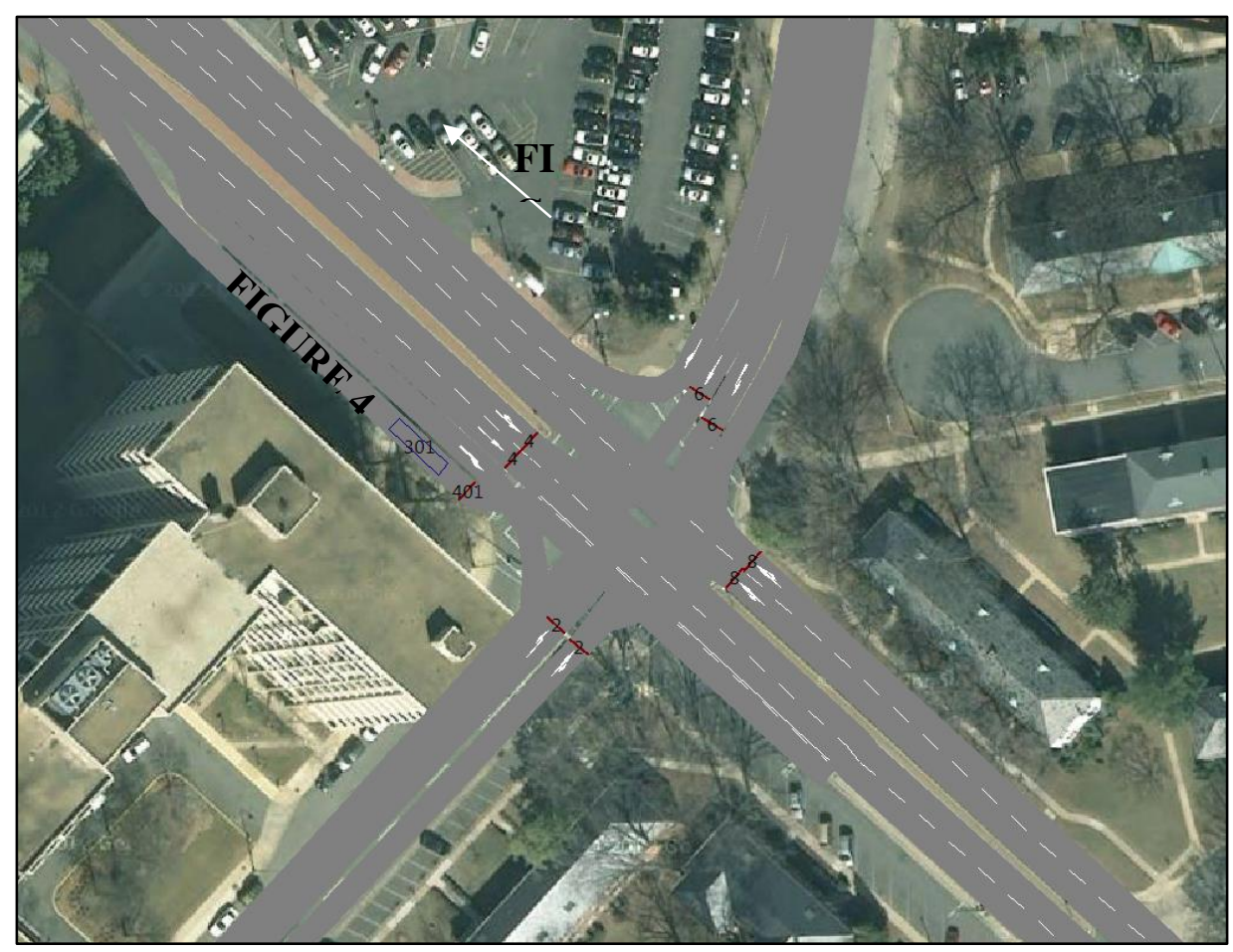

Figure 1 Study site geometry

\section{SCENARIO DESIGN}

As noted, this study assesses existing queue jumper bus stop in Arlington County and to compare it with Connected Vehicle (CV) and no queue jumper lane case. CV allows the bus to communicate with the traffic controller when it is ready to leave, which would be beneficial if the bus stop is at a traffic light. These two scenarios are to be compared to a control of queue jumper lane without the transit signal priority. The goal is to test how beneficial queue jumper lanes are when paired with phase insertion signal priority, and to test whether or not utilizing CV with queue jumper lanes is feasible.

The third objective involves removing the extra queue jumper lane and installing queue jumper lanes in right-hand turn lanes. Since not many intersections are with dual right turn lane, and making geometry changes is costly. It is our goal to achieve a better understanding whether combining the only existing right turn lane with a queue jumper lane is beneficial enough to warrant widespread implementation. In this model, both buses and right-hand turning traffic share the lane. This model is designed to emulate current transit bus stop setup, and to measure the effectiveness of making the right turn lane into a queue jumper lane as well. While this model no longer resembles Glebe and Henderson, it is a decent replica of stops around the country. When the right-hand turn lane is also used as a queue jumper lane, the right-hand turning vehicles receive an early green as well if there is a bus in the queue. Ideally, the bus would move to the front of the queue because of vehicles turning right on red. Our research will 
determine how beneficial it is to turn an existing right turn lane into a right turn and queue jumper combined lanes.

The following is a detailed description of all five scenarios tested. In short, the first three scenarios are based on the existing road geometry in Arlington County, while the rest two scenarios are under different road geometry, or the intersection with the queue jumper lane removed.

The first scenario represents the current conventional jumper lane bus stop as it currently is. The general algorithm for this scenario is that at the beginning of every cycle, right before the south bound direction traffic light turns green. If a bus is at the front of the jumper lane, an early green of 7 seconds is given. No early green is awarded when no bus is detected. This scenario is referred to as normal queue jumper bus stop (Normal).

The second scenario is the proposed enhanced queue jumper bus stop with the addition of the Connected Vehicle (CV) technology. The objective of this advanced mechanism is not only to reduce bus delays, but also to reduce the delays that queue jumper lanes cause to other vehicles. In this scenario, with CV technology, two-way communications between a bus and traffic signal controller is taken advantage of. When a bus arrives at the bus stop, it estimates its dwell time based on potential passenger count at the bus stop, and transmits this information to the signal. Then the signal will decide whether to grant queue jumper green to guarantee that a bus does not receive an early green unless it is ready to go. Also, when there are multiple buses requesting for queue jumper green, the algorithm increases the green length ( 3 seconds more per bus in this study) according to the number of requests. Theoretically, this queue jumper with $\mathrm{CV}$ algorithm potentially prevents a bus from impeded by the queue accumulating during the head start green for bus (if the bus is dwelling and does not take advantage of the head start). This algorithm also saves time for following buses when multiple buses come at the same time and minimize unnecessary delay caused to general traffic. This scenario is referred as queue jumper with $\mathrm{CV}$ $(\mathrm{CV})$.

The third scenario is a control scenario, or the queue jumper bus stop setup without a transit signal priority. This assesses how beneficial giving transit buses phase insertion is when a queue jumper lane already exists. The objective of this scenario is to determine the magnitude bus delay is reduced by the addition of the signal priority. This scenario is referred as no queue jumper (NO).

The fourth scenario is another control. It is the baseline for the fifth scenario and is essentially a normal intersection with a bus stop in the right turn lane. The goal of this scenario is to imitate as many current near-side stops as possible. This scenario is referred to as joint lane without queue jumper (JL_NO).

The final scenario is an innovative alternative design of queu jumper bus stop. It is a modified version of the fourth scenario with the addition of queue jumper function on the right turn lane. Its purposes are to measure the effectiveness of a queue jumper lane when it is used in a right turn lane, and to determine whether it would be beneficial enough compared to adding an extra queue jumper lane. Assuming queue jumper lane with signal priority decreases delays both 
"with extra lane" and "without extra lane", it is important to measure which improves more and by how much magnitude. This scenario is referred to as joint lane with queue jumper (JL).

\section{EVALUATION METHOD}

A stochastic simulation model-based evaluation was performed to accommodate the randomness in the real world. A microscopic simulation tool VISSIM was utilized to conduct a performance evaluation on all five scenarios. (11) The simulation network was an isolated intersection based on the Glebe and Henderson intersection in Arlington County, VA,. USA. Traffic volume and bus schedule data were collected from the study site. Volume utilized was the morning peak hour data. The signal timing plan adopted was directly from the study site that only reflected the current volume with a queue jumper lane. It had not been optimized for other volume scenarios and bus headways evaluated in this study. Future research may consider the change in timing plan with the change in traffic volumes, bus headways, and the existence of shared queue jumper lane. The model had been calibrated to represent the real world. It was achieved by adjusting the car following model parameters to reach a realistic saturation flow rate at the intersection. The model was visually examined by the project manager at the division of transportation in Arlington, VA to ensure the validity of the simulation. In order to access all the information that will be available in a connected vehicles environment, such as identification number, coordinates, speeds, and acceleration, a COM interface was added into the VISSIM model. The COM interface was the tool that made changes in signal phasing in real time during simulation (12).

To consider the effect of simulation randomness, 10 simulation runs were performed for each scenario and the MOEs for each scenario were averaged from the output of each of the 10 runs. Minimum sample size requirement was checked to make sure that sufficient number of simulation runs was achieved to represent the entire population. Minimum sample size was calculated using formula recommended by the Virginia Department of Transportation (13) , which is:

$$
\mathrm{N}=Z^{2} * S_{S}^{2} /\left(X_{S} * E\right)^{2}
$$

Where,

Z: Number of standard deviations away from the mean corresponding to the required confidence level in a normal distribution. In this study, confidence level is set to be $95 \%$.

Ss: Sample standard deviation.

Xs: Sample mean.

E: Tolerable error. In this study, $\mathrm{E}=10 \%$.

The study intended to evaluate the performance of a queue jumper lane in terms of both bus driver's perspective and general traffic's perspective. Therefore, performance measures adopted are bus delay and total delay at the intersection. Sensitivity studies had been conducted on three parameters: congestion level (i.e., v/c=0.35, 0.50, 0.7 and 0.9), headway (i.e., $3 \mathrm{~min}, 5 \mathrm{~min}, 9$ min and $15 \mathrm{~min}$ ), and right turn volume (i.e., 108, 308, 508, and $708 \mathrm{vph}$ ). To be noted, only one parameter varied at a time. For example, when conducting sensitivity study on congestion level, bus arrival time followed strictly the bus schedule on site. When headway level varies, the volume was the current peak hour volume at the intersection. 
Finally, all the differences had been checked for statistical significance. The purpose was to ensure that all the improvements or adverse effects claimed in the result session are statistically significant. Paired t-test with two tailed was utilized, since data in comparison was collected from the same site. Confidence level tested was $95 \%$.

\section{RESULTS}

The performances of the 5 different designs of queue jumper bus stop were compared in this section. They were compared in terms of bus delay and total delay at the intersection. Various congestion levels, bus headway and right turn volumes were tested to reveal the performance of QJL under different traffic conditions.

\section{Congestion Level}

Various congestion levels were tested here to gain better understanding of the performances of different queue jumper bus stop designs. The results are shown in Figure 2. Overall, queue jumper with $\mathrm{CV}(\mathrm{CV})$ showed the best performance under various congestion levels. Compared to no queue jumper condition (NO), it saved bus delay time about $30 \%$. Compared to existing queue jumper bus stop at Arlington County, the CV saved bus delay about $15 \%$. The results also proved the value of the existing queue jumper bus stop at Arlington County (i.e., Normal condition). In general, existing queue jumper saved bus delay about $15 \%$. Joint lane with queue jumper (JL) showed no statistically significant disadvantage over normal queue jumper lane (Normal) when $\mathrm{v} / \mathrm{c}$ ratio is under 0.7. It means adding an extra lane for queue jumper purpose provides no extra benefit if the site of interest is not highly congested. However, if congestion level gets close to capacity, queue jump lane fails to function and give normal queue jumper an advantage of about $13 \%$ bus delay saving. This finding is consistent with Zhou (7) that signal priority without an auxiliary lane would be less effective under congested traffic. Finally, Figure 2 shows that bus delay for NO condition and JL_NO condition demonstrated no statistical significance throughout various congestion levels. This result also tells that, if without queue jumper function, adding a bus bay would have very minor influence on bus' travel time. 


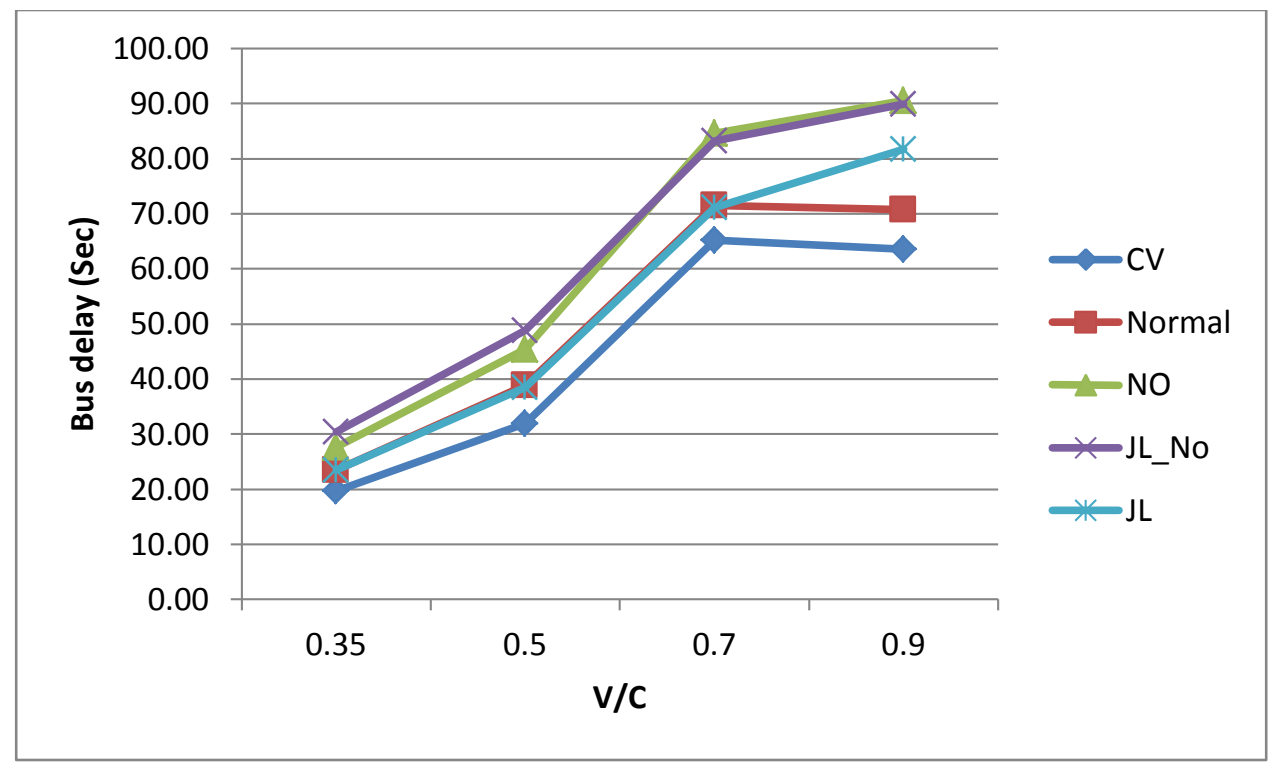

Figure 2 Bus delay with various congestion levels.

No statistically significant difference was found on total delay of general traffic among all five scenarios. The hypothesis that these five scenarios have the same total delay cannot be rejected at a 95\% confidence level. The results are shown in Figure 3 . Therefore, adding queue jumper function had very little impact on general traffic.

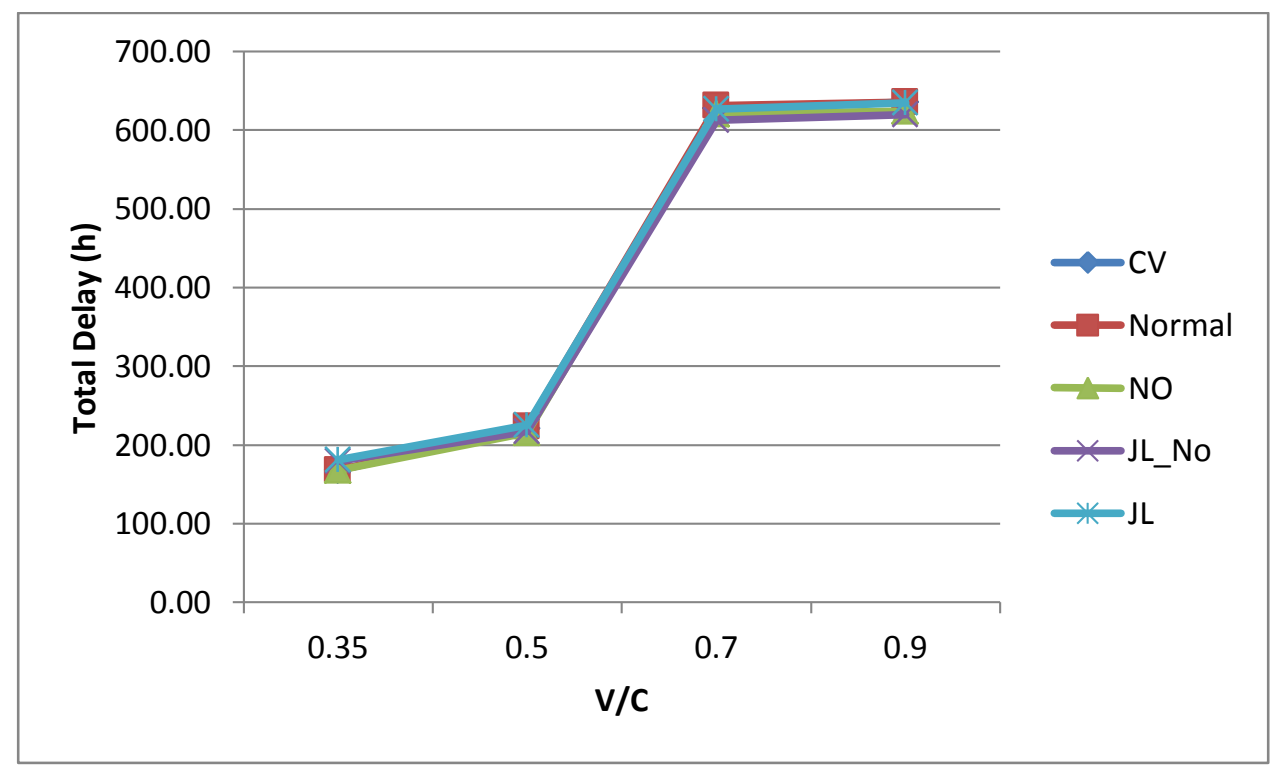

Figure 3 Total delay with various congestion levels.

\section{Headway}

The research investigated the performance of different queue jumper bus stops under various headways. Four headway levels were tested: $3 \mathrm{~min}, 5 \mathrm{~min}, 9 \mathrm{~min}$ and $15 \mathrm{~min}$. The results are presented in Figure 4 and Figure 5. Overall, headway did not impact much on bus delay, but did 
impact total delay of all traffic users at the intersection. It makes sense because the smaller the headway between buses the more interference would be cast on general traffic.

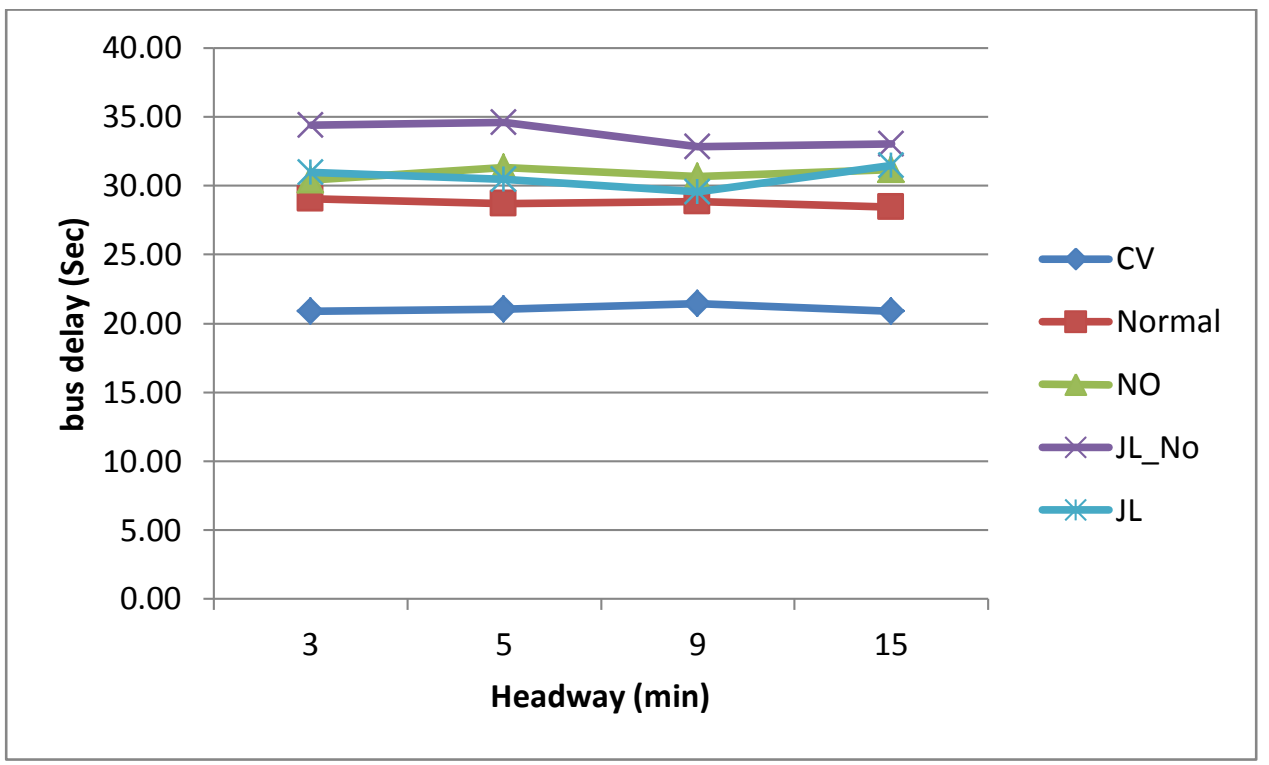

Figure 4 Bus delay with various headway.

Most scenarios have consistent performance throughout various headway levels, except for joint lane with queue jumper scenario. When headway was around 9 min, which is the current headway at the intersection, JL showed similar performance as normal queue jumper. However, when headway increases or decreases, the benefit of JL would decrease accordingly. It is possible that for JL, there is an optimal headway level for bus.

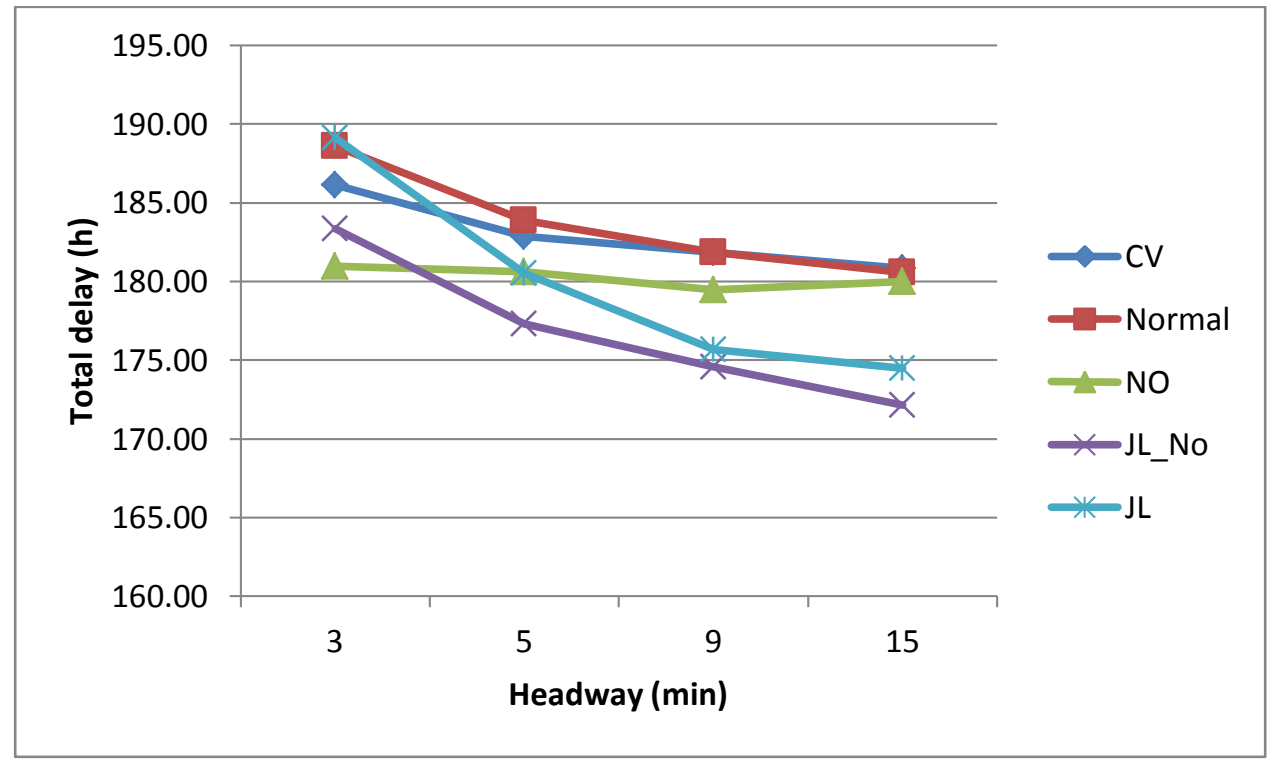

Figure 5 Total delay with various headway. 
For no queue jumper scenario (NO), headway showed no impact on total delay of all traffic users. While other scenarios showed a negative correlation between total delay and bus headway level. As shown in Figure 5, the Normal scenario caused the most interference on general traffic. With $\mathrm{CV}$, total delay was reduced. However, paired t-test revealed that the differences among Normal, $\mathrm{NO}$ and $\mathrm{CV}$ were not statistically significant across all headway levels. Therefore, both conventional queue jumper bus stop (Normal) and queue jumper with $\mathrm{CV}(\mathrm{CV})$ caused no adverse effect on general traffic. To be noted, although all five designs are plotted here in the same figure, Normal is not the baseline for JL because of their difference in geometry. JL should be compared against JL_No. As observed in Figure 5, JL caused minor increase on total delay across all headway levels, except when headway equals to 9 minutes, the other three differences are statistically significant.

\section{Right Turn Volume}

For joint lane scenarios, since buses are using the same lane as the right turning vehicles, it is possible that the queue jumper function would be influenced by right turn volume. Therefore, various right turn volume levels were tested. Four volume levels investigated are: 108 vph, 308 vph, $508 \mathrm{vph}$, and $708 \mathrm{vph}$.

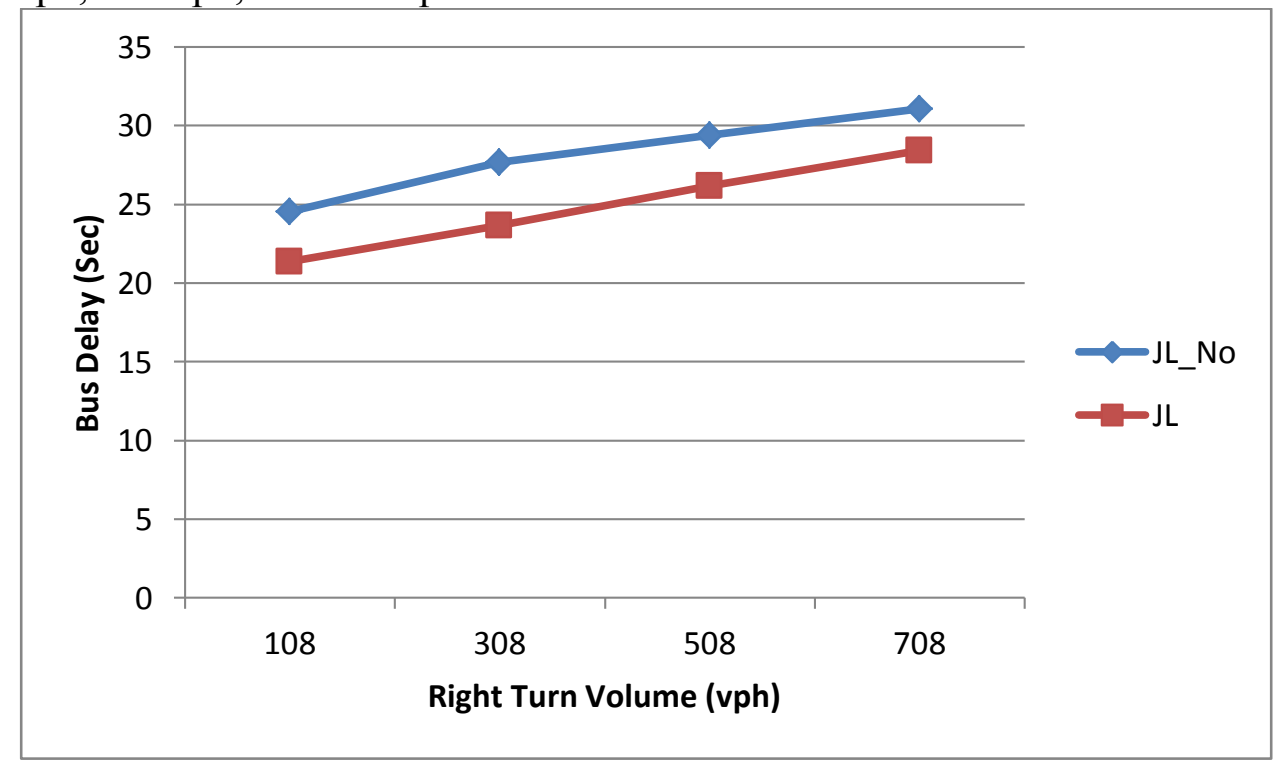

\section{Figure 6 Bus delay with various right turn.}

The result showed that the benefit of joint lane with queue jumper demonstrates a negative correlation with right turn volume. As right turn volume increases from 108 vph to $708 \mathrm{vhp}$, the delay saving drops from $15 \%$ to $9 \%$. Nonetheless, constantly, JL is statistically beneficial to transit buses. 


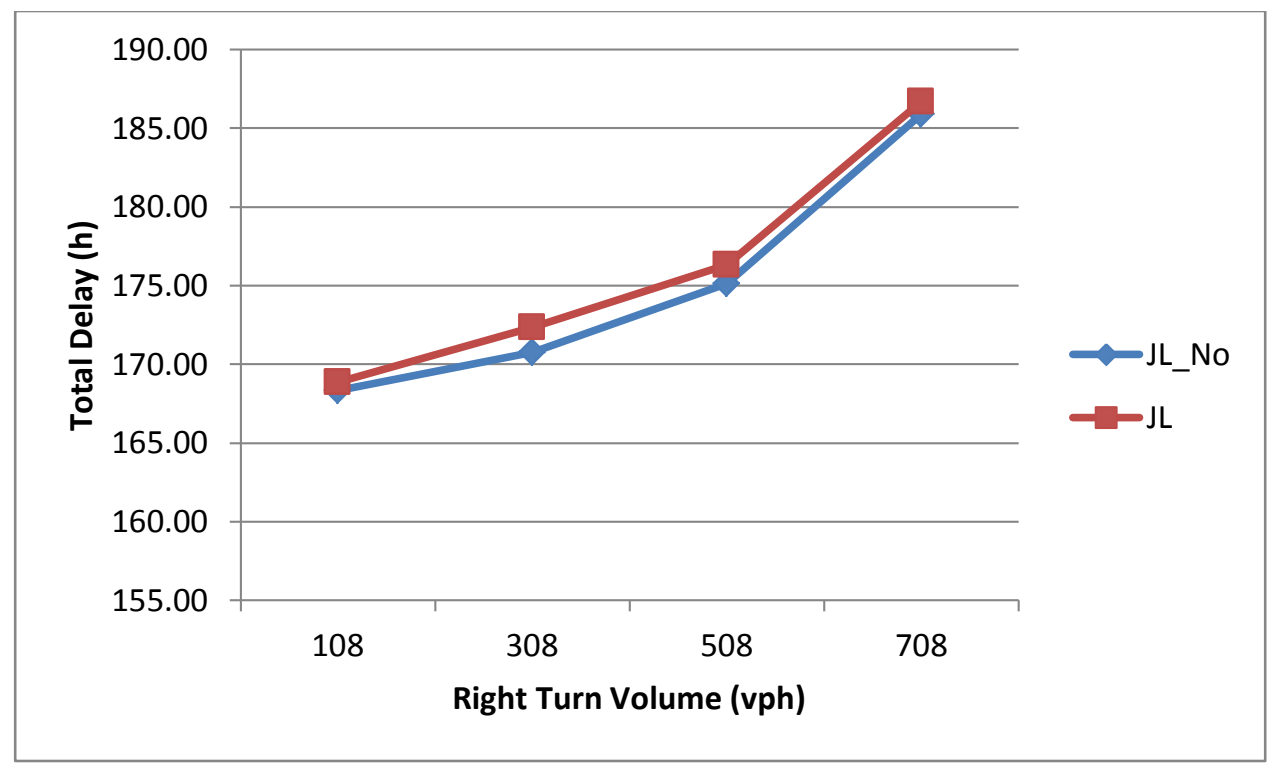

Figure 7 Total delay with various right turn.

Joint lane with queue jumper did not increase total delay. The hypothesis that joint lane with and without queue jumper share the same total delay cannot be rejected at a 95\% confidence level.

\section{CONCLUSIONS AND FUTURE RESEARCH}

Transit buses at a near-side bus stop often suffer from significant time loss when trying to merge back into traffic. Given that most bus stops in urban areas within the US are near-sided, it is important to help bus merge back to traffic stream more efficiently. Instead of moving near-side bus stops to far-side, an alternative solution is to add a queue jumper lane to near-side bus stop. This paper investigated different designs of queue jumper lane bus stop and proposed a new queue jumper bus stop design utilizing connected vehicle technology.

The study confirmed the value of the existing queue jumper bus stop in Arlington County area and demonstrated via simulation that the existing queue jumper near-side bus stop in Arlington County causes no adverse effect on general traffic users. Therefore, in case the bus fleet is not equipped with CV technology, it is a good option to add an extra queue jumper lane to near-side bus stop and pair it with head-start green time. A $15 \%$ reduction on bus delay is observed even without connected vehicle technology.

Queue jumper bus stop with CV technology is the most recommended way of adding queue jumper lane to near-side bus stop. The results demonstrated that queue jumper with $\mathrm{CV}$ technology produced the best benefit of about 30\% delay reduction for buses. Queue jumper with $\mathrm{CV}$ produced constant benefit over various congestion levels and bus headway scenarios. In addition, queue jumper with $\mathrm{CV}$ did not cause extra delay to other traffic users. It is important to note that an extra queue jumper lane is required to secure consistent bus delay savings under various volume levels. Otherwise, time saving would diminish when the intersection is at near congestion. 
In case, the geometry on a road does not allow an extra lane for queue jumper or budget is limited, this paper tested an alternative to turn the right turn lane into a joint lane of right turn and queue jumper lane. It is discovered that joint lane with queue jumper would function just as well as queue jumper with extra lane when the road is not congested and headway between buses is about 9 minutes. In addition, the simulation results showed that right turn volume does have a negative correlation with queue jumper lane performance. But joint queue jumper lane is always beneficial under various right turn volume levels, even though queue jumper with joint lane is sensitive to bus headway. When headway deviates from its optimal level of about 9 minutes, the benefit of joint-lane queue-jumper lane diminished and eventually failed when traffic is close to congestion.

The following recommendaionts were made for future research. Firstly, this study investigated the benefit of adding a queue jumper lane to a near-side bus stop at an isolated intersection. Thus, future research should look into the effect on progression of a whole corridor. Secondly, the signal timing plan used in this study was not optimized for other volume scenarios and bus headways evaluated in this study. Future research should use optimized timing plans with varying traffic volumes and bus headways.

\section{ACKNOWLEDGEMENTS}

The authors are grateful to Ms. Bing Zhang and Chris Copeland of the University of Virginia who helped building initial VISSIM network and evaluating Arlington County queue jumper bus stop. This research was in part supported by Connected Vehicle/Infrastructure University Transportation Center. 


\section{REFERENCES}

1. Jason Werner. "Houston Metro Plans to Deploy a More Intelligent Transit Priority Scheme," National Associations Working Group for ITS, 2002.

2. Matt Johnson. "Relocate Bus Stops to Save Time," http://greatergreaterwashington.org/post/4636/relocate-bus-stops-to-save-time/, Accessed July 5, 2013.

3. Peter B. Ohlms. "Strategies for Transit Priority," Preceedings of $1^{\text {st }}$ National Conference on Intermodal Transportation: Problems, Practices, and Policies, Oct 2012, pp. pp 34-46.

4. Gu Weihua, Michael J Cassidy, Vikash VGayah, and Yanfeng Ouyang. "Mitigating Negative Impacts of Near-Side Bus Stops on Cars," Transportation Research Part B: Methodological, Vol. 47, 2013, pp. pp 42-56.

5. Brossard KHONDAKER and SC (Chan) WIRASINGHE. "Bus Stop Spacing and Location for a Corridor with Multiple Bus Routes," The Many Faces of Transportation, 2013, pp. 13 p.

6. Marcos Medina-Tapia, Ricardo Giesen, and Juan Munoz. "A Model for the Optimal Location of Bus Stops and its Application to a Public Transport Corridor in Santiago," Presented at the $92^{\text {nd }}$ Annual Meeting of the Transportation Research Board, Washington, D.C.., 2013, pp. 20p.

7. Guangwei Zhou, and Albert Gan. "Design of Transit Signal Priority at Signalized Intersections with Queue Jumper Lanes," Journal of Public Transportation, Vol. 12, No. 4, 2009, pp. pp 117132.

8. Guangwei Zhou, Albert Gan, and Xiaoxia Zhu. "Determination of Optimal Detector Location for Transit Signal Priority with Queue Jumper Lanes," Transportation Research Record: Journal of the Transportation Research Board, No. 1978, 2006, pp. pp 123-129.

9. An Zhou. "Queue Jumper Lanes: A Strategy for Improving Transit Performance?" http://www.itegoldcoast.org/scholarshiparticles/Queue\%20Jumper\%20Lanes\%20\%20A\%20Strategy\%20for\%20Improving\%20Transit\%20Performance.pdf, Accessed July 1, 2013.

10. Guangwei Zhou, and Albert Gan. "Performance of Transit Signal Priority with Queue Jumper Lanes," Transportation Research Record: Journal of the Transportation Research Board, No. 1925, 2005, pp. pp 265-271.

11. PTV. VISSIM 5.10 User Manual., Jul. 2008.

12. PTV. VISSIM 5.10-03 COM Interface Manual., Sep. 2008. 
13. VDOT Traffic Engineering Division. "Traffic Operations Analysis Tool Guidebook," http://www.virginiadot.org/business/resources/traffic_engineering/VDOT_Traffic_Operations_A nalysis_Tool_GuidebookV1.1-August2013.pdf, Accessed Dec 15, 2013. 


\title{
INTELLIGENT TRANSIT SIGNAL PRIORITY CONSIDERING CONFLICTING REQUESTS
}

By

\author{
$\mathrm{Jia} \mathrm{Hu}^{*}, \mathbf{1 )}$ \\ Ph.D. Candidate
}

Tel: 919-744-9842, Email: jh8dn@virginia.edu

\section{Byungkyu "Brian" Park, Ph.D. ${ }^{1)}$}

Associate Professor

Tel: (434) 924-6347, Email: bpark@,virginia.edu

\section{Young-Jae Lee, Ph.D. ${ }^{2)}$}

Associate Professor

Tel: (443) 885-1872, Email: YoungJae.Lee@morgan.edu

4) Department of Civil and Environmental Engineering University of Virginia

P.O. Box 400742

Charlottesville, VA 22904-4742

Fax: (434) 982-2951

5) Department of Transportation and Urban Infrastructure Studies

Morgan State University

1700 E. Cold Spring Lane, Baltimore, Maryland 21251

Fax: (443) 885-8218

July 2014

Submitted for consideration for presentation at the $94^{\text {th }}$ Annual Meeting of the Transportation Research Board, January 12-16, 2015

Word Count: 5,487 text words plus 2,000 for figures/tables $(8 * 250)=7,487$ total

*Corresponding Author 


\section{ABSTRACT}

Transit Signal Priority (TSP) has been studied as a control strategy that offers preferences to transit vehicles at signalized intersections. This paper presents an enhanced TSP logic based on Connected Vehicle (CV) technology to address challenges of the previously developed TSPCV mechanism. The newly strengthened logic, referred to as Transit Signal Priority with Connected Vehicle Technology Accommodating Multiple Buses (TSPCVM), is capable of accommodating multiple TSP requests by taking advantage of the two-way communications and additional and more accurate information enabled from Connected Vehicle technology.

The logic is evaluated in two ways. An analytical approach identifies theoretical improvement by conducting an exhaustive search to find the best TSP timing plan. A microscopic simulation approach provides an assessment of realistic improvements of the TSP logic. It is compared with no TSP (NTSP) and conventional TSP (CTSP) under different scenarios. The measures of effectiveness used are bus delay and total travel time of all travelers. The performance of TSPCVM is also compared against conventional TSP (CTSP) under various congestion levels. The analytical results show that the TSPCVM greatly reduces bus delay at signalized intersection for all congestion levels considered. A microscopic traffic simulationbased evaluation results also showed that the TSPCVM logic reduces the bus delay between 5\% and $48 \%$ compared to the conventional TSP. The range of improvement corresponding to four different $\mathrm{v} / \mathrm{c}$ ratios tested, which are $0.5,0.7,0.9$ and 1.0. No negative effects on other traffic users are observed except when $\mathrm{v} / \mathrm{c}=1.0$. 


\section{INTRODUCTION}

Transit Signal Priority (TSP) has been applied in many cities in Europe, Asia and North America (1-3). It refers to a variety of strategies that provide preferences to transit buses at signalized intersections. It is believed that TSP could reduce bus delay, improve schedule adherence, and increase road network mobility.

When a transit bus sends out a request as it approaches a traffic signal controlled intersection, the conventional TSP responds with a simple extension to or early start of the originally planned green time (2-4). To decide which logic should be used, a quick calculation is performed on site using a bus arrival time model based on historical data. If the bus is expected to arrive shortly before the original green time, the green time starts early; and vice versa (5). This kind of TSP logic is restricted in many ways. Most importantly, because the data fed into the model is either outdated or not accurate, the bus arrival time forecast could be severely biased. The inaccurate forecast of the bus arrival time could waste extra TSP green time and cause unnecessary adverse effects on side streets. Additionally, even with extension and/or early start, conventional TSP green time can only cover a small portion of a traffic signal cycle. Therefore, a large portion of buses may not benefit from the TSP.

To properly address these challenges, a next generation TSP logic based on Connected Vehicle technology (TSPCV) was proposed in (6). This new TSP takes advantage of the resources provided by Connected Vehicle (CV) technology, including two-way communications between the bus and the traffic signal controller, accurate bus location detection and prediction and the number of passengers. The key feature of the proposed TSP logic is the green time reallocation, which moves green time instead of adding extra green time. The TSP was also designed to be conditional. That is, the delay per person served as the most important criterion determining whether TSP is to be granted. Based on the simulation results, the proposed TSP was shown to provide buses with more accuracy and better effectiveness. Furthermore, it accommodates a higher percentage of transit buses than conventional TSP. Its performance was compared against the conventional TSP (CTSP) and no-TSP (NTSP) conditions under various congestion levels. The results show that the TSPCV greatly reduces the bus delay at signalized intersections without causing statistically significant negative effects on side streets.

Like many other advanced TSP strategies $(1,7-9)$, the TSPCV was developed to accommodate one single bus at a time. In case that multiple conflicting TSP requests were made, the system was designed to serve the first request only. Nevertheless, it was discovered that the current "first come, first serve" way of solving conflicting priority requests not only does no benefit but also deteriorates the TSP system. A 13\% extra bus delay was observed with firstcome-first-serve strategy compared to no-TSP option (10).

Very few studies have investigated the problem of conflicting TSP requests. Ma et al. developed two methods accommodating multiple TSP requests, the first is a passive bus priority for exclusive bus lane that maximizes person capacity (11), the other is using decision tree to 
decide serve sequence (12). He et al. presented a heuristic algorithm which reduces up to $50 \%$ of the bus delay compared to the "first come first serve" policy (13). Zlatkovic et al. proposed a logic which always provides priority first to the direction with the green phase on (10). This algorithm shows a benefit of more than $30 \%$ reduction on traffic light delay. Both algorithms have room to be improved. First of all, He's algorithm is designed and generate significant benefit only during over saturated condition. Secondly, they were both developed for the condition when only two strategies are applied: "green extension" and "red truncation" (5). These algorithms are not applicable towards complicated TSP strategies like the previously developed TSPCV logic. Finally, they also lack consideration regarding adverse effect on other traffic users. Hence, an enhanced TSPCV logic is required which accommodates conflicting TSP requests while causing no negative effect to other traffic users.

\section{RESEARCH OBJECTIVE}

The objectives of this paper are as follows:

1. Enhance the previously developed TSPCV logic in the sense of accommodating multiple conflicting TSP requests. Since the enhanced TSPCV is capable of dealing with multiple TSP requests, to distinguish from the previous TSPCV logic, the new logic will be referred to as TSPCVM.

2. Evaluate the newly upgraded TSPCVM logic.

The remainder of this paper describes the structure of the TSPCVM, presents analytical and simulation-based evaluation results and findings, and identifies the contributions.

\section{LOGIC ARCHITECTURE DESCRIPTION}

The TSPCVM logic is activated every time a bus sends out a TSP request. When the TSPCVM logic is called, it will check two criteria before proceeding to TSP timing plan computation. The first criterion checks whether the bus is behind schedule and the second one verifies whether this TSP request conflicts with any previously accepted request. If the first criterion is not met, then no TSP is granted. If the second criterion is not fulfilled, then the logic degrades into the previously developed TSPCV logic. The bus will be accommodated through the single TSP request mechanism which is TSPCV. In summary, since the proposed TSP logic is built upon the previously developed TSPCV logic, several core characteristics are inherited from the previous logic. First, the cooperation between transit buses and traffic signals is required and enabled. When a bus approaching an intersection sends a priority request, not only the traffic controller tries to accommodate the buses, but also the bus needs to travel at a reasonable speed to minimize the effort from the signal. The bus speed is recommended based on remaining/expected queue, road geometry and normal signal timing plan. Secondly, the TSP logic proposed is the green time reallocation, in other words, instead of adding additional green time to the original timing plan the proposed TSP logic splits the original green time and moves part of it to when green time is mostly needed by a transit bus. Finally, TSP green time is granted conditionally based on two criteria which are schedule adherence and delay per person. Many aforementioned functions are made possible by the Connected Vehicle (CV) technology, including two-way communications between the bus and the traffic signal controller, accurate bus location detection and prediction, and the number of passengers. More specific descriptions of the TSPCVM logic 
are discussed in this section. FIGURE displays the architecture of the TSPCVM. The logic is composed of three major components:

1. Arrival time prediction component, in which arrival time ranges of all buses approaching the subject intersection are predicted.

2. TSP timing plan and bus speed calculation component, in which, given the arrival time ranges, the algorithm generates a timing plan that will have minimum impact on general traffic users and calculates corresponding recommended bus speeds.

3. Logic assessment and implementation component, in which the TSP timing plan will be compared against the normal signal time (winner overwrites the other) and the recommended bus speeds will be transmitted to the coming buses.

Detailed information regarding the three components of TSPCV is provided in the literature (6). The following presents the logic of TSPCVM.

\section{Arrival Time Prediction Component}

Every and each time a bus approaches the intersection, this component is activated and predicts the arrival time for all the buses that are traveling towards the intersection. One of the great advantages of integrating TSP into a CV system is the two-way communications between road side equipment (RSE) and traffic users which in this case is the bus. A CV-equipped bus communicates with traffic signal controllers and is capable of receiving speed instructions. It is assumed that the desired speed of an approaching bus could vary between $10 \%$ above and $20 \%$ below the speed limit. Therefore, the prediction result generated from this component is not a set of fixed numbers; instead, it is a set of time ranges. The ranges of arrival time are computed for all the approaching buses for the next step so that the buses can adjust their travel at various speeds to cooperate with the TSP strategy.

\section{TSP Timing Plan and Bus Speed Calculation Component}

This component is called right after the previous arrival time prediction component. It identifies all buses approaching the intersection and judges whether these TSP requests are conflicting. Conflicting TSP requests are defined as multiple TSP green time requested within one signal cycle. If no conflicting request is detected, the logic degrade into the single bus TSPCV logic, and otherwise, it proceeds to compute the potential TSP green time for all buses. The potential TSP green time is calculated based on the goal that TSP green is inserted exactly where it is needed for the duration it is needed. The duration of the TSP green time is determined so that the queue is cleared before the bus arrives at the intersection. In other words, the bus will catch up with the end of the queue right at the stop bar of the intersection. The calculation of the real-time queue length estimation is based on the model developed by Liu (14) which is an extension of the shock wave theory. The arrival information of other vehicles at the end of the queue is acquired using connected vehicle technology. 


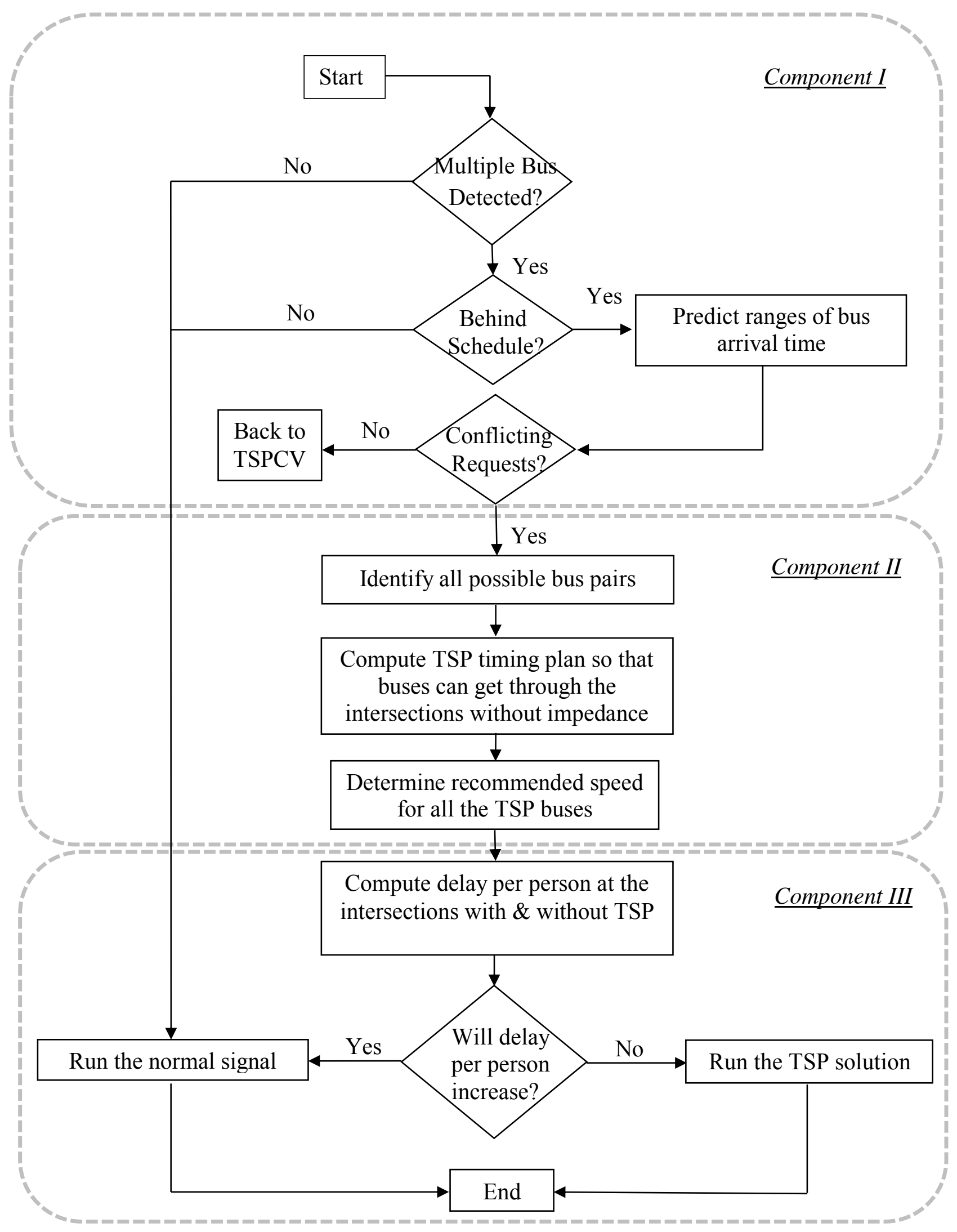

FIGURE 1 The Structure of TSPCVM 
The cycle length will be the same even when the TSP green is inserted, because the TSP green time is spliced from the original green time in the direction of the bus. The inserted green time taken from the certain direction is $100 \%$ used in clearing the traffic for that direction. Therefore, theoretically speaking, not a single second is wasted during the TSP. So strictly speaking, the extra TSP green time is "moved" rather than "inserted" or "added." This feature greatly minimizes the adverse effect compared to the conventional TSP which adds extra green that no other traffic users except for bus can make use of.

The ranges of predicted bus arrival time are passed on from the last step for TSP timing plan calculation. Therefore, the corresponding potential TSP green time the computation finds is a group of ranges of TSP green start time and end time. The number of ranges equals to the number of conflicting TSP requests. While there will be numerous TSP timing plans depending on when the buses arrive, the identified potential TSP green time is filtered firstly using the following rules:

1. A maximum of $2 \mathrm{TSP}$ green time should be granted within one signal cycle. If 3 or more conflicting TSP requests are made, the algorithm identifies all possible 2-bus pairs and then accommodates the bus pair associating with the least travel time of all vehicles.

2. If two conflicting TSP buses are traveling towards each other from opposite directions, it is preferred they travel through the intersection within one single TSP green time.

3. A TSP green time is preferred to start at the end of phase rather than to cut into the middle of a non-TSP signal phase (for better safety and drivers' expectation).

4. If a TSP green has to start in the middle of a phase, it is preferred that the bus travels at its normal speed.

5. Minimum green time is required for both the TSP green time and the original timing plan.

Based on these rules, the algorithm finds a pool of preferred TSP starts and ends times from the time range that TSP can possibly start and end while fitting them into an optimization algorithm. This optimization algorithm is activated every time a TSP request is received, it finds the value of choice variables $T_{T S P e n d i}$ to minimize total person delay at the intersection for a preset time interval. The objective function estimating total person delay can be expressed as follows:

The choice variables include: $T_{T S P e n d 1}, T_{T S P e n d 2} \ldots T_{T S P e n d k}$.

$$
\operatorname{Minimize}\left(\sum_{c y c l e=1}^{c y c l e=\mathrm{n}} \int_{0}^{T} D_{i} * O c c_{i}+\sum_{K=1}^{k} \sum_{c y c l e=1}^{\text {cycle }=\mathrm{n}} \int_{0}^{T} D_{b u s_{-} k} * O c c_{b_{u s} \_}\right)
$$

Which subject to:

$$
\begin{gathered}
\sum_{j}\left(G_{j_{-} \text {before } \_k}+G_{j_{-} \text {after } \_}\right)+G_{T S P_{-} k}+G_{\text {remain } \_k}=T=\text { constant } \\
T_{\text {TSPend_k }}-T_{T S P S_{-} k}=G_{T S P_{-} k} \\
T_{\text {BAlow } k} \leq T_{T S P e n d_{-} k} \leq T_{\text {BAup } \_k}
\end{gathered}
$$




$$
\begin{aligned}
& T_{T S P S_{-} k}=\sum_{j} G_{j \_b e f o r e \_k} \\
& G_{j_{\_} b e f o r e \_k}+G_{j_{\_} a f t e r \_k}=\text { Constant } \\
& G_{j_{-} \text {before } k} \geq G_{\min } \text { or } G_{j_{-} \text {before } k}=0 \\
& G_{j_{-} a f t e r_{-} k} \geq G_{\min } \text { or } G_{j_{-} a f t e r_{-} k}=0 \\
& G_{T S P_{-} k} \geq G_{\min } \text { or } G_{T S P_{-} k}=0 \\
& G_{\text {remain_k }} \geq G_{\min } \text { or } G_{\text {remain } \_k}=0
\end{aligned}
$$

Where:

$k$ : The number of conflicting TSP requests

$T$ : cycle length at the intersection

$D i$ : Delay of vehicle i

$D_{\text {bus } \_ \text {: }}$ : Delay of bus k

$O c c_{i}$ : Occupancy on vehicle i

$O c c_{\text {bus_k }}$ : Occupancy on the bus k

$G_{j \_b e f o r e \_}$: Green time for phase $\mathrm{j}(1,2$, or 3$)$ before TSP green granted for bus k

$G_{j_{a} a f t e r \_}$: Green time for phase $\mathrm{j}(1,2$, or 3$)$ after TSP green granted for bus k

$G_{T S P_{-} k}$ : TSP green time granted for bus $\mathrm{k}$

$G_{\text {remain_k}}$ : Remaining green time for lane group with bus after taking out the TSP green granted

for bus k

$G_{m i n}$ : minimum green time requirement

$T_{T S P e n d \_k}$ : End time of TSP green granted for bus $\mathrm{k}$

$T_{T S P S_{-} k}:$ Start time of TSP green granted for bus $\mathrm{k}$

$T_{B A l o w \_}$: Lower bound of bus arrival time range of bus $\mathrm{k}$

$T_{B A u p \_k}$ : Upper bound of bus arrival time range of bus $\mathrm{k}$

The optimization algorithm finds a set of TSP green time associated with the least delay of all vehicles. Computation power is not an issue here. Based on the case study performed, the algorithm can finish within a second. Therefore, implementing this logic on a real time basis is feasible. Once the timing plan is generated, the recommended bus speeds are computed so that buses travel through the intersection right after the queues in front are cleared and before TSP green phases end.

\section{Logic Assessment and Implementation Component}

After a TSP timing plan is determined, the algorithm compares the "with TSP" scenario against the "normal timing" scenario. Since the number of passengers on board is likely to be known under the CV environment, person delay performance measure is to be used. The person delay is calculated for a number of consecutive signal cycles starting from the TSP implemented cycle. In this study, a TSP timing plan is only implemented when its corresponding person delay is less than the "no TSP" scenario. 
During implementation, two major steps are conducted. First, an instruction is given to a bus about the desired recommended speed. Second, the signal time is altered to accommodate TSP green. A possible buffer green time is given to a bus in case a bus is not expected to make it to the intersection. The TSP green time can be extended up to 5 seconds to accommodate the random delay.

\section{EVALUATIONS \\ Study Site}

The test network is a VISSIM model of the intersection at Emmet St. and Barracks Rd. in Charlottesville, VA, as shown in Figure 2. The model had been calibrated to match the real world. It was achieved by adjusting the car following model parameters to reach a realistic saturation flow rate at the intersection. The model was also visually examined by the researcher to ensure the validity of the simulation. Current signal cycle at the intersection is 160 seconds.

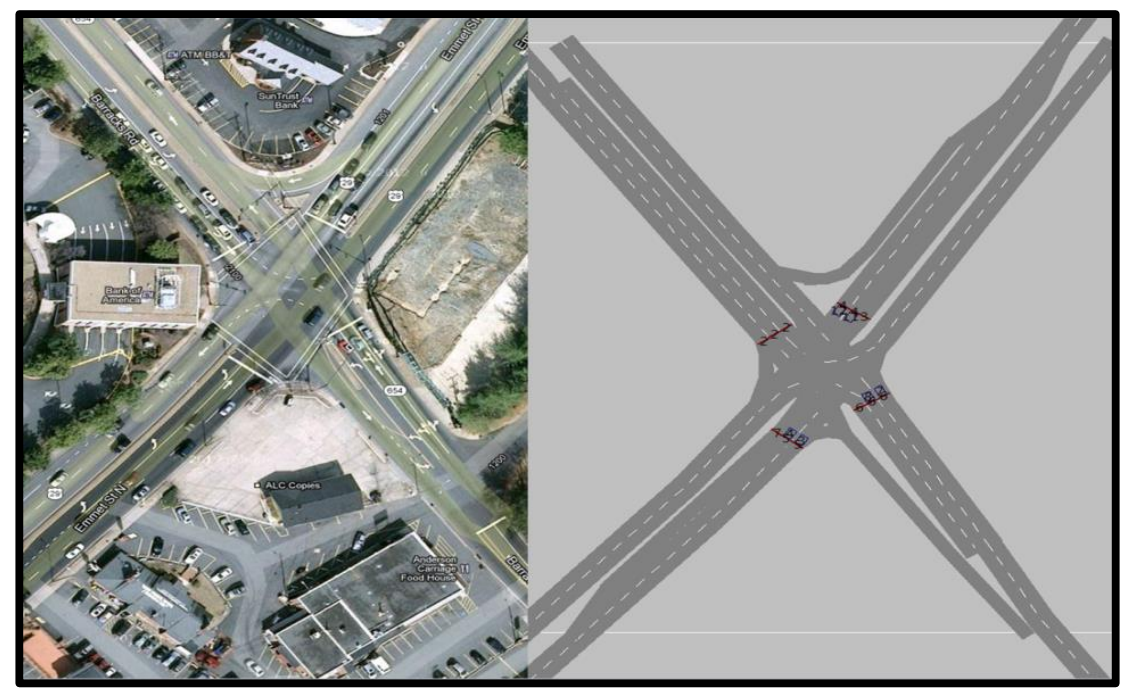

\section{FIGURE 2 Study site-Emmet St and Barracks Rd intersection, Charlottesville.}

\section{Methodology}

Both analytical tests and simulation evaluations have been performed for the proposed TSPCVM as well as conventional TSP (CTSP) and no TSP (NTSP) cases. The analytical test is based on a deterministic calculation. It considers all the possible conflicting scenarios and quantifies the performance by averaging the measurement of effectiveness computed for all these scenarios. On the other hand, simulation evaluation considers uncertainty due to vehicle interactions and interarrival times. This evaluation better mimics real world and quantifies the performance more realistically. Both evaluation methods are essential in order to achieve a comprehensive understanding of the logic. The conventional TSP logic compared here is the first-come-first serve TSP. It is not only because the first-come-first-serve TSP is the current common practice, but also because all the aforementioned research use the FCFS TSP as the control group. By comparing to the same benchmark, the advantage of TSPCVM is clearly presented. The CTSP grants 10 -second extra green time to buses which arrive within 10 seconds of the end of normal 
green time. In case the bus could not make through the intersection within that 10 seconds, CTSP will add to the previous 10 seconds with up to 5 seconds to accommodate the late arrival. When multiple TSP requests are made within one signal cycle, only one request is accommodated by the CTSP. The logic follows the real implementation in the Northern Virginia (15).

All three logic cases are investigated under three scenarios:

1. Two conflicting requests from opposite directions

2. Two conflicting requests from perpendicular directions

3. Three conflicting requests from three directions

The research assumes buses are traveling towards the intersection from three different directions. All the bus lines are with mid-block bus stops located about 750 feet upstream of the intersection. With the speed limit of $40 \mathrm{mph}$, it is assumed buses can travel within the speed range between $30 \mathrm{mph}$ and $45 \mathrm{mph}$. The TSP logic is activated when buses pass 0.5 mile upstream of the intersection. The optimization algorithm minimizes the total person delay at the intersection for 3 signal cycles. It is noted that three cycles are used to be long enough to capture residual effects caused by TSP and be short enough not to interfere with another TSP. Three 160second cycles are about the minimum headway between buses. The buses traveling in the system are assumed with 40 riders and their average dwell time at the bus stops is 30 seconds with 2 seconds standard deviation. These attributes regarding the buses are obtained from an NCHRP study investigating bus rapid transit (16). The bus dwell time variation is set moderately low, since the CV technology improves dwell time prediction and minimizes forecast deviation.

The measures of effectiveness (MOE) used are bus delay and total travel time of all travelers. Bus delay quantifies the effectiveness of various TSP treatments while the total travel time demonstrates whether any adverse effect is caused.

To consider the effect of simulation randomness, at least 10 simulation runs were performed for each scenario and the MOEs for each scenario were averaged from the output of all simulation runs. Minimum sample size requirement was checked to make sure that sufficient number of simulation runs was achieved to represent the entire population. Minimum sample size was calculated using the formula recommended by the Virginia Department of Transportation (17), which is:

Where,

$$
\mathrm{N}=Z^{2} * S_{s}^{2} /\left(X_{S} * E\right)^{2}
$$

$Z$ : Number of standard deviations away from the mean corresponding to the required confidence level in a normal distribution. In this study, confidence level is set to be $95 \%$.

Ss: Sample standard deviation.

$X s$ : Sample mean.

$E$ : Tolerable error. In this study, $\mathrm{E}=10 \%$.

Finally, all the differences have been checked for statistical significance. The purpose is to ensure that all the improvements or adverse effects claimed in the result session are 
statistically significant. Paired two tailed T-test with was utilized, since data in comparison was collected from the same site. Confidence level tested was $95 \%$.

\section{Analytical Test}

In order to prove the benefit of TSPCVM on a theoretical level, the performance of TSPCVM accommodating two conflicting TSP requests is estimated first using analytical analysis. Stop delay of buses and all other traffic users is calculated using the deterministic computation. In order for a fair evaluation, all possible TSP activation scenarios need to be considered and averaged to find the assessment results. Given the cycle length at the intersection is 160 seconds. Since a TSP can be activated at any given second, and two bus lines are under consideration, there are $160 * 160=25600$ possible TSP activation situations. Stop delay for bus and all other traffic users is calculated by averaging these $160 * 160$ situations. All three treatments have been computed and compared. Field peak-hour volume data is applied, which is near capacity situation $(\mathrm{v} / \mathrm{c}=0.9)$. The results are summarized in the Table 1.

TABLE 1 Analytical Assessment on Various TSP Treatments

\begin{tabular}{ccccccc}
\hline & NTSP & CTSP & ITSP & N/TSP & N/C & C/TSP \\
\hline $\begin{array}{c}\text { Delay Per Person } \\
\text { (Opp) (Sec) }\end{array}$ & 49.5 & 49.2 & 44.3 & $10.5 \%$ & $0.8 \%$ & $9.8 \%$ \\
$\begin{array}{c}\text { Delay Per Person } \\
\text { (Perp) (Sec) }\end{array}$ & 49.5 & 49.2 & 46.0 & $7.2 \%$ & $0.7 \%$ & $6.5 \%$ \\
$\begin{array}{c}\text { Bus Delay } \\
\text { (Opp) (Sec) }\end{array}$ & 105.4 & 99.2 & 51.8 & $50.9 \%$ & $5.9 \%$ & $47.8 \%$ \\
$\begin{array}{c}\text { Bus Delay } \\
\text { (Perp) (Sec) }\end{array}$ & 117.2 & 109.4 & 65.4 & $44.2 \%$ & $6.6 \%$ & $40.3 \%$ \\
\hline
\end{tabular}

Table 1 presents the performance of TSPCVM accommodating 2 different kinds of conflicting TSP requests: two buses coming from opposite directions (Opp) and buses coming from perpendicular directions (Perp). Both per person delay of all traffic users and bus delay are compared. It is discovered that TSPCVM is superior over CTSP regardless of conflict conditions tested in this evaluation. While CTSP showed comparable benefits over two conflicting scenarios, TSPCVM demonstrated more advantages when buses are coming from opposite directions. Not only the bus delay is reduced more, delay per person is also minimized in a greater magnitude. This observation is intuitive because two opposite traveling buses can be accommodated in a single TSP green. As a result, more bus passengers are provided with preference at the same time while fewer disturbances are caused to other traffic users. To further prove this hypothesis, Table 2 is computed. 
TABLE 2 TSP Granting Condition

\begin{tabular}{ccccc}
\hline Perp & Main St & Minor St & Both Buses & None \\
\hline count & 13800 & 10828 & 972 & 0 \\
\% & $53.9 \%$ & $42.3 \%$ & $3.8 \%$ & $0.0 \%$ \\
Opp & Main St 1 & Main St 2 & Both Buses & None \\
count & 11032 & 9460 & 5108 & 0 \\
\% & $43.1 \%$ & $37.0 \%$ & $20.0 \%$ & $0.0 \%$ \\
\hline
\end{tabular}

Table 2 presents the number of TSPCVM granted sorted by TSP type out of the $160 * 160$ different conflicting conditions. The first column is the number of TSP granted only to the bus traveling on the main street. The second column represents the number of TSP granted only to the other bus. The third column shows the number of cases that both buses receive TSP. As shown in Table 2, the likelihood of double accommodation for opposite traveling buses is $20 \%$ which is about 4 times higher than that of the perpendicular traveling buses. This is the reason why TSPCVM demonstrates more advantage when buses are coming from opposite directions. In addition, the results also reveal that the TSPCVM logic prefers buses traveling on the direction with a higher volume level. As a larger portion of buses on the main street are granted with TSP than that of the buses on the minor street. Given that the volume is slightly higher on the main street 1 direction than the main street 2 direction, the percentages associated demonstrate the same relationship. It is intuitive because the TSP green not only provides preference to the buses, but also let pass the vehicles that are traveling with the bus. The higher the volume, the greater the benefit will the other traffic users experience, hence the more likelihood will this TSP plan be executed.

\section{Simulation-based Evaluation in VISSIM}

While the analytical test results show significant benefits under the proposed TSPCVM logic, it does not consider any variability due to vehicle interactions and inter-arrival times. A microscopic traffic simulator can assess the performance of the proposed TSP under more plausible conditions. The microscopic simulation software package VISSIM (18) is used to evaluate the proposed TSP logic under a Connected Vehicle environment. A COM interface is used to assess information that is available within a Connected Vehicle environment (19). The evaluation is performed under the assumption that only transit buses are connected to traffic signal controllers and other traffic users do not have $\mathrm{CV}$ equipment. In other words, $0 \% \mathrm{CV}$ market penetration except for buses. The end of queue is estimated based on incoming vehicles and outgoing vehicles at the intersection. Detailed algorithm can be found in the model developed by Liu (14) which is an extension of the shock wave theory. Therefore, the data extracted via COM interface is only speed and position of bus, number of passengers on board, number of potential passenger at the bus stop, number of vehicles passing the intersection and volume from all four approaches. Also, the COM interface is used to change signal timing plan during the simulation. All programs are coded in Microsoft EXCEL VBA. 
The simulation test network has been calibrated to better match the real world.

Measurement utilized is saturation flow rate. In order to reduce the saturation flow rate to a realistic range, the default settings of Wiedemann 74 car following model have been adjusted. Average standstill distance is raised to 7.5 , additive part to 3 and multiplicative part to 4 . After the parameters' adjustments, saturation flow rate is reduced to an average of $1838 \mathrm{veh} / \mathrm{h} / \mathrm{ln}$. It is consistent with the HCM 2010 which states that the saturation flow rate on an urban street segment is $1800 \mathrm{veh} / \mathrm{h} / \mathrm{ln}(20)$.

The simulation based evaluations compared all three scenarios:

1. Two conflicting requests from opposite directions

2. Two conflicting requests from perpendicular directions

3. Three conflicting requests from three directions

Each scenario ran at least 10 times with different random seeds. It is ensured that the results show statistical significance with a 95\% confidence level and 5\% tolerance error. The sample size sufficiency is assessed using the formula recommended by the Virginia Department of Transportation (17).

All the differences shown in the following tables have been checked for statistical significance. The differences that are NOT significant are underlined and in italics. All other changes were determined to be statistically significant at $\alpha=0.05$.

\section{Conflicting Requests From Opposite Directions}

As noted, the test network is a calibrated model of the intersection at Emmet St. and Barracks Rd. in Charlottesville, VA. Vehicle volumes and turning movements are actual morning peak-hour data collected from the site. Bus dwelling time at the stop is 30 seconds average with a standard deviation of 2 seconds. A pair of transit buses is designed to arrive every 494 seconds. Given the cycle length is 160 seconds at the intersection; the interval of bus arrival is exactly 3 cycles plus 14 seconds. Also, the headway between the two buses within a pair increases by 14 seconds every time another pair of buses is generated. This research purposefully designed the offset and headway to be 14 seconds so that buses within one single simulation run will arrive at different times relative to signal cycles; hence the simulation result would be less biased.

The results from the simulation based evaluation are shown in TABLE a. Bus delay and total travel time of all vehicles were summarized and averaged from all simulation runs. The proposed TSP treatment was compared with NTSP and CTSP conditions and T-test was performed to validate that all the differences are statistically significant. In sum, the simulation based results support the findings from the analytical analysis. Compared to the other scenarios, most significant improvements are observed. The delay of all buses is reduced by $44 \%$ compared to CTSP and 50\% compared to NTSP. Delay of all traffic users is slightly minimized as well. An unbalance of improvement is observed between the two bus lines. Partially, as aforementioned, it is because the traffic volume travelling the same direction with the second bus line is less. As a result, the algorithm tends to provide more preference for the bus line 1 . The other reason is that 
a larger portion of buses on line 2 arrives during the green phase. Hence, the room for improvement is less compared to line 1 .

TABLE 3a Two Conflicting Requests from Opposite Directions

\begin{tabular}{cccccc}
\hline & TSPCVM & CTSP & NTSP & ITSP/CTSP & ITSP/NTSP \\
\hline $\begin{array}{c}\text { Bus 1 Delay } \\
\text { (Sec) }\end{array}$ & 16.4 & 38.3 & 46.6 & $57.3 \%$ & $64.9 \%$ \\
$\begin{array}{c}\text { Bus 2 Delay } \\
\text { (Sec) }\end{array}$ & 24.2 & 34.2 & 34.4 & $29.1 \%$ & $29.5 \%$ \\
$\begin{array}{c}\text { Total Bus } \\
\text { Delay (Sec) }\end{array}$ & 40.6 & 72.5 & 81.0 & $44.0 \%$ & $49.8 \%$ \\
$\begin{array}{c}\text { Total Travel } \\
\text { Time (Sec) }\end{array}$ & 579802.7 & 612359.3 & 613774.1 & $-5.3 \%$ & $-5.5 \%$ \\
\hline
\end{tabular}

\section{Conflicting Requests from Perpendicular Directions}

The setting of simulation here is mostly the same as the previous scenario, except that the buses are now coming from perpendicular directions. Again this research purposefully designed the offset and headway to be 14 seconds so that buses within one single simulation run will arrive at different times relative to signal cycles; hence the simulation result would be less biased.

The results from the simulation based evaluation are shown in Table $3 \mathrm{~b}$. Bus delay and total travel time of all vehicles are summarized and averaged from all simulation runs. The proposed TSP treatment is compared with NTSP and CTSP conditions and t-test was performed. In summary, the simulation based results support the findings from the analytical analysis. The delay of all buses is reduced by $31 \%$ compared to CTSP and 35\% compared to NTSP.

Regardless of the fact that the delay of all traffic users raises slightly, the differences are proven to be statistically insignificant. Interestingly, buses on the minor street are showing larger improvement than the buses on the principal street. One major reason is that a larger room of improvement exists for the minor street buses. Therefore, when being granted with TSP, minor street buses tend to generate more delay savings. Hence, despite that less portion of minor street buses receive TSP, more benefit is observed. 
TABLE 3b Two Conflicting Requests from Perpendicular Directions

\begin{tabular}{cccccc}
\hline & TSPCVM & CTSP & NTSP & ITSP/CTSP & ITSP/NTSP \\
\hline $\begin{array}{c}\text { Main St Bus } \\
\text { Delay (Sec) } \\
\text { Minor St }\end{array}$ & 42.0 & 52.7 & 53.4 & $20.4 \%$ & $21.5 \%$ \\
$\begin{array}{c}\text { Bus Delay } \\
\text { (Sec) }\end{array}$ & 40.6 & 66.9 & 73.2 & $39.4 \%$ & $44.6 \%$ \\
$\begin{array}{c}\text { Total Bus } \\
\text { Delay (Sec) }\end{array}$ & 82.5 & 119.6 & 126.6 & $31.0 \%$ & $34.8 \%$ \\
$\begin{array}{c}\text { Total Travel } \\
\text { Time (Sec) }\end{array}$ & 551599.7 & 530469.6 & 533278.0 & $\underline{4.0 \%}$ & $\underline{3.4 \%}$ \\
\hline
\end{tabular}

Conflicting Requests from Three Directions

The setting of simulation here is mostly consistent as the previous scenario, except that one more bus line is included in the consideration. The same bus schedule generated in the previous two scenarios is adopted. The consideration again is to ensure that buses within one single simulation run will arrive at different times relative to signal cycles; hence the simulation result would be less biased.

The results from the simulation based evaluation are shown in Table $3 \mathrm{c}$. The magnitude of improvement is not as significant as the two-conflicting-requests conditions. It is because the nature of TSPCVM only allows a maximum of two TSP grants at a time. As a result, when more than two TSP requests are made, at least one bus does not receive TSP treatment. Although this fact reduces the size of improvement, the results are still showing $18 \%$ reduction in bus delay compared to CTSP and $21 \%$ drop compared to NTSP. Again no adverse effect on other traffic users is caused by TSPCVM. Nevertheless, the TSPCVM does show advantage over CTSP on total travel time of all traffic users.

TABLE 3c Conflicting Requests from Three Directions

\begin{tabular}{cccccc}
\hline & TSPCVM & CTSP & NTSP & ITSP/CTSP & ITSP/NTSP \\
\hline $\begin{array}{c}\text { Main St Bus 1 } \\
\text { Delay (Sec) }\end{array}$ & 41.7 & 46.1 & 49.9 & $9.5 \%$ & $16.5 \%$ \\
$\begin{array}{c}\text { Main St Bus 2 } \\
\text { Delay (Sec) }\end{array}$ & 32.5 & 34.5 & 34.7 & $5.9 \%$ & $6.5 \%$ \\
$\begin{array}{c}\text { Minor St Bus } \\
\text { Delay (Sec) }\end{array}$ & 50.6 & 70.8 & 72.9 & $28.5 \%$ & $30.6 \%$ \\
$\begin{array}{c}\text { Total Bus } \\
\text { Delay (Sec) }\end{array}$ & 124.8 & 151.3 & 157.5 & $17.6 \%$ & $20.8 \%$ \\
$\begin{array}{c}\text { Total Travel } \\
\text { Time (Sec) }\end{array}$ & 608602.9 & 617539.6 & 613305.6 & $-1.4 \%$ & $\underline{-0.8 \%}$ \\
\hline
\end{tabular}




\section{Sensitivity Analysis on Congestion Levels}

In order to verify that the findings from the experiment are consistent with various congestion levels, a sensitivity analysis is conducted comparing TSPCVM against CTSP. Since the field collected volume data is at $\mathrm{v} / \mathrm{c}$ ratio 0.9 , three other scenarios are tested: $\mathrm{v} / \mathrm{c}=0.5, \mathrm{v} / \mathrm{c}=0.7$ and $\mathrm{v} / \mathrm{c}=1.0$. The results have been presented in Table $4 \mathrm{a}$ through Table $6 \mathrm{~b}$.

All three scenarios show similar trends with respect to how TSPCVM performs under various congestion levels. When the congestion level is low, TSPCVM helps reduce bus delays up to about $44 \%$ compared to CTSP. As the congestion level rises, the benefit of TSPCVM decreases, while no extra delay is caused. This is because the algorithm is designed to be conditional on person delay. When the volume becomes closer to the capacity, less portion of the green time will be granted to TSPCVM to prevent TSP from causing extra delay on other travelers. As a result, the benefit will drop correspondingly, while adverse effects on side streets would still be kept under a certain level. It is interesting to see that even when $\mathrm{v} / \mathrm{c}$ ratio equals to 0.9 , the benefit of TSPCVM is still significant and drops dramatically when $\mathrm{v} / \mathrm{c}$ becomes 1.0 . However, even when $\mathrm{v} / \mathrm{c}=1.0$, TSPCV is still superior to conventional TSP. Furthermore, the TSPCVM logic only shows unbalanced preference under near capacity conditions. When $\mathrm{v} / \mathrm{c}$ level is low, the improvements observed by different bus line are similar. As the congestion level rises, the difference in delay saving rises with it. Sometimes, the logic would even sacrifice one bus line in order to achieve an overall delay reduction.

As shown in Table $4 \mathrm{a}$ and Table $4 \mathrm{~b}$, the greatest delay reduction is observed when conflicting TSP requests come from opposite directions. The performance of TSPCVM reacts to congestion level change the same fashion as described above. Statistically significant total travel time reduction is observed under all congestion levels tested. 
TABLE 4a Sensitive Analysis for Conflicting Requests from Opposite Directions (Bus Delay)

\begin{tabular}{|c|c|c|c|c|c|}
\hline & $\mathbf{v} / \mathbf{c}=$ & 0.5 & 0.7 & 0.9 & 1.0 \\
\hline \multirow[t]{3}{*}{ TSPCVM } & $\begin{array}{c}\text { Main St } \\
\text { Bus 1 Delay } \\
\text { (Sec) }\end{array}$ & 18.1 & 16.9 & 16.4 & 33.0 \\
\hline & $\begin{array}{l}\text { Main St } \\
\text { Bus } 2 \text { Delay } \\
\text { (Sec) }\end{array}$ & 17.1 & 17.1 & 24.2 & 47.2 \\
\hline & $\begin{array}{l}\text { Total Bus } \\
\text { Delay (Sec) }\end{array}$ & 35.2 & 34.1 & 40.6 & 80.2 \\
\hline \multirow[t]{3}{*}{ CTSP } & $\begin{array}{c}\text { Main St } \\
\text { Bus } 1 \text { Delay } \\
\text { (Sec) }\end{array}$ & 32.5 & 35.4 & 38.3 & 47.1 \\
\hline & $\begin{array}{c}\text { Main St } \\
\text { Bus } 2 \text { Delay } \\
(\mathrm{Sec})\end{array}$ & 28.2 & 29.7 & 34.2 & 50.5 \\
\hline & $\begin{array}{l}\text { Total Bus } \\
\text { Delay }(\mathrm{Sec})\end{array}$ & 60.7 & 65.1 & 72.5 & 97.7 \\
\hline \multirow[t]{3}{*}{ Improvement } & $\begin{array}{c}\text { Main St } \\
\text { Bus 1 Delay } \\
(\mathrm{Sec})\end{array}$ & $44.4 \%$ & $52.2 \%$ & $57.3 \%$ & $30.1 \%$ \\
\hline & $\begin{array}{c}\text { Main St } \\
\text { Bus } 2 \text { Delay } \\
\text { (Sec) }\end{array}$ & $39.3 \%$ & $42.4 \%$ & $29.1 \%$ & $6.5 \%$ \\
\hline & $\begin{array}{l}\text { Total Bus } \\
\text { Delay }(\mathrm{Sec})\end{array}$ & $42.0 \%$ & $47.7 \%$ & $44.0 \%$ & $17.9 \%$ \\
\hline
\end{tabular}

TABLE 4b Sensitive Analysis for Conflicting Requests from Opposite Directions (Total Delay)

\begin{tabular}{ccccc}
\hline v/c & $\begin{array}{c}\text { TSPCVM } \\
\text { (h) }\end{array}$ & $\begin{array}{c}\text { CTSP } \\
\text { (h) }\end{array}$ & Diff & t-test \\
\hline $\mathbf{0 . 5}$ & 91.7 & 93.8 & $2.3 \%$ & $6.80 \mathrm{E}-07$ \\
$\mathbf{0 . 7}$ & 122.8 & 128.5 & $4.4 \%$ & $7.81 \mathrm{E}-05$ \\
$\mathbf{0 . 9}$ & 161.1 & 170.1 & $5.3 \%$ & $1.84 \mathrm{E}-07$ \\
$\mathbf{1 . 0}$ & 197.3 & 204.1 & $3.3 \%$ & $3.00 \mathrm{E}-02$ \\
\hline
\end{tabular}

Table $5 \mathrm{a}$ and Table $5 \mathrm{~b}$ demonstrate how bus delay savings and total travel time change with congestion level when conflicting TSP requests come from perpendicular directions. The performance of TSPCVM mostly reacts to congestion level change the same fashion as described above. When the volume is at the capacity, the logic sacrifices the bus line on the main street to achieve overall bus delay improvement. It is discovered that TSPCVM reduces delay for other 
traffic users when $\mathrm{v} / \mathrm{c}=0.5$. No statistically significant adverse effect is observed under all other congestion levels.

TABLE 5a Sensitive Analysis for Conflicting Requests from Perpendicular Directions (Bus Delay)

\begin{tabular}{|c|c|c|c|c|c|}
\hline & $\mathbf{v} / \mathbf{c}=$ & 0.5 & 0.7 & 0.9 & 1.0 \\
\hline \multirow[t]{3}{*}{ TSPCVM } & $\begin{array}{c}\text { Main St } \\
\text { Bus Delay } \\
\text { (Sec) }\end{array}$ & 24.6 & 28.9 & 42.0 & 71.7 \\
\hline & $\begin{array}{l}\text { Minor St } \\
\text { Bus Delay } \\
\text { (Sec) }\end{array}$ & 40.0 & 41.5 & 40.6 & 43.7 \\
\hline & $\begin{array}{c}\text { Total Bus } \\
\text { Delay (Sec) }\end{array}$ & 64.6 & 70.4 & 82.5 & 115.4 \\
\hline \multirow[t]{3}{*}{ CTSP } & $\begin{array}{l}\text { Main St } \\
\text { Bus Delay } \\
\text { (Sec) }\end{array}$ & 43.7 & 47.6 & 52.7 & 54.5 \\
\hline & $\begin{array}{c}\text { Minor St } \\
\text { Bus Delay } \\
\text { (Sec) }\end{array}$ & 63.9 & 65.8 & 66.9 & 66.9 \\
\hline & $\begin{array}{l}\text { Total Bus } \\
\text { Delay }(\mathrm{Sec})\end{array}$ & 107.6 & 113.4 & 119.6 & 121.4 \\
\hline \multirow[t]{3}{*}{ Improvement } & $\begin{array}{l}\text { Main St } \\
\text { Bus Delay } \\
\text { (Sec) }\end{array}$ & $43.7 \%$ & $39.4 \%$ & $20.4 \%$ & $-31.5 \%$ \\
\hline & $\begin{array}{l}\text { Minor St } \\
\text { Bus Delay } \\
\text { (Sec) }\end{array}$ & $37.4 \%$ & $36.9 \%$ & $39.4 \%$ & $34.6 \%$ \\
\hline & $\begin{array}{c}\text { Total Bus } \\
\text { Delay (Sec) }\end{array}$ & $39.9 \%$ & $38.0 \%$ & $31.0 \%$ & $5.0 \%$ \\
\hline
\end{tabular}

TABLE 5b Sensitive Analysis for Conflicting Requests from Perpendicular Directions (Total Delay)

\begin{tabular}{ccccc}
\hline $\mathbf{v} / \mathbf{c}=$ & $\begin{array}{c}\text { TSPCVM } \\
\text { (h) }\end{array}$ & $\begin{array}{c}\text { CTSP } \\
\text { (h) }\end{array}$ & Diff & t-test \\
\hline $\mathbf{0 . 5}$ & 92.9 & 96.0 & $3.3 \%$ & 0.0001 \\
$\mathbf{0 . 7}$ & 132.3 & 132.2 & $\underline{-0.1 \%}$ & 0.8938 \\
$\mathbf{0 . 9}$ & 179.9 & 173.0 & $\underline{-4.0 \%}$ & 0.0821 \\
$\mathbf{1 . 0}$ & 206.6 & 206.7 & $\underline{\underline{0.1 \%}}$ & 0.9849 \\
\hline
\end{tabular}

Table $6 \mathrm{a}$ and Table $6 \mathrm{~b}$ demonstrate how bus delay savings and total travel time change with congestion level under three conflicting TSP requests scenario. The performance of TSPCVM mostly reacts to congestion level change the same fashion as described above. When 
the volume is at the capacity, the logic sacrifices the bus line 1 on the main street to achieve overall bus delay improvement. No statistically significant adverse effect is observed under all congestion levels.

TABLE 6a Sensitive Analysis for Conflicting Requests from Three Directions (Bus Delay)

\begin{tabular}{|c|c|c|c|c|c|}
\hline & $\mathrm{v} / \mathrm{c}=$ & 0.5 & 0.7 & 0.9 & 1.0 \\
\hline \multirow[t]{4}{*}{ TSPCVM } & $\begin{array}{c}\text { Main St } \\
\text { Bus } 1 \text { Delay } \\
\text { (Sec) }\end{array}$ & 33.3 & 37.2 & 41.7 & 57.8 \\
\hline & $\begin{array}{c}\text { Main St } \\
\text { Bus } 2 \text { Delay } \\
\text { (Sec) }\end{array}$ & 25.9 & 26.8 & 32.5 & 47.5 \\
\hline & $\begin{array}{l}\text { Minor St } \\
\text { Bus Delay } \\
\quad(\mathrm{Sec})\end{array}$ & 53.3 & 55.1 & 50.6 & 54.3 \\
\hline & $\begin{array}{c}\text { Total Bus } \\
\text { Delay }(\mathrm{Sec})\end{array}$ & 112.4 & 119.1 & 124.8 & 159.6 \\
\hline \multirow[t]{4}{*}{ CTSP } & $\begin{array}{c}\text { Main St } \\
\text { Bus } 1 \text { Delay } \\
\text { (Sec) }\end{array}$ & 36.8 & 40.8 & 46.3 & 51.4 \\
\hline & $\begin{array}{c}\text { Main St } \\
\text { Bus } 2 \text { Delay } \\
\text { (Sec) }\end{array}$ & 27.6 & 29.7 & 34.7 & 51.4 \\
\hline & $\begin{array}{l}\text { Minor St } \\
\text { Bus Delay } \\
\quad(\mathrm{Sec})\end{array}$ & 64.0 & 66.0 & 70.0 & 67.4 \\
\hline & $\begin{array}{c}\text { Total Bus } \\
\text { Delay (Sec) }\end{array}$ & 124.6 & 136.5 & 151.0 & 170.2 \\
\hline \multirow[t]{4}{*}{ Improvement } & $\begin{array}{c}\text { Main St } \\
\text { Bus } 1 \text { Delay } \\
\text { (Sec) }\end{array}$ & $9.6 \%$ & $8.9 \%$ & $10.1 \%$ & $-12.4 \%$ \\
\hline & $\begin{array}{c}\text { Main St } \\
\text { Bus } 2 \text { Delay } \\
\text { (Sec) }\end{array}$ & $6.4 \%$ & $9.8 \%$ & $6.4 \%$ & $7.6 \%$ \\
\hline & $\begin{array}{c}\text { Minor St } \\
\text { Bus Delay } \\
\quad(\mathrm{Sec})\end{array}$ & $16.7 \%$ & $16.5 \%$ & $27.7 \%$ & $19.4 \%$ \\
\hline & $\begin{array}{c}\text { Total Bus } \\
\text { Delay }(\mathrm{Sec})\end{array}$ & $9.8 \%$ & $12.8 \%$ & $17.4 \%$ & $6.2 \%$ \\
\hline
\end{tabular}


TABLE 6b Sensitive Analysis for Conflicting Requests from Three Directions (Total Delay)

\begin{tabular}{ccccc}
\hline $\mathbf{v} / \mathbf{c}=$ & $\begin{array}{c}\text { TSPCVM } \\
(\mathbf{h})\end{array}$ & $\begin{array}{c}\text { CTSP } \\
\text { (h) }\end{array}$ & Diff & t-test \\
\hline 0.5 & 84.2 & 87.3 & $3.5 \%$ & 0.046 \\
0.7 & 116.2 & 120.3 & $3.4 \%$ & 0.020 \\
0.9 & 169.1 & 171.8 & $1.6 \%$ & 0.088 \\
1.0 & 188.4 & 188.2 & $\underline{-0.1 \%}$ & 0.642 \\
\hline
\end{tabular}

\section{CONCLUSIONS AND FUTURE RESEARCH}

The research fills in the knowledge gap and provides methods to resolve multiple conflicting TSP requests at an isolated intersection. It overcomes the challenge bore by the conventional "first come first serve" strategy and presents significant improvement on bus service performance. At the same time, the logic also minimizes the interruption caused by providing TSP green time, showing promise in reducing cost to implement the system.

The enhanced TSPCVM logic proposed was built upon the foundation of the previously developed TSPCV logic. It inherits the merits from the TSPCV which are vehicle-infrastructure cooperation and green time reallocation. These two features greatly increase the portion of TSP accommodated buses and minimize unused TSP green time. In addition, the improved TSP, taking advantage of the two-way communications and additional and more accurate information provided by Connected Vehicle technology, is capable of accommodating multiple conflicting TSP requests. The logic incorporates an algorithm that prioritizes the buses coming from different approaches and solves for the premium signal timing that minimizes the total delay at the intersection. By determining the total number and sequence of the buses accommodated, the most bus delay is saved while the least total delay of all motorists is achieved. Both analytical tests and simulation evaluations have been performed to evaluate the TSPCVM logic. The results show that, when under moderate volume condition, bus delay is reduced by approximately 40 $50 \%$. Furthermore, its performance is compared against conventional TSP (CTSP) conditions under various congestion levels and various conflicting conditions. Results demonstrate that the TSPCVM logic reduces the bus delay between $5 \%$ and $48 \%$ compared to the conventional TSP. The range of improvement corresponding to four different $\mathrm{v} / \mathrm{c}$ ratios is tested, which are $0.5,0.7$, 0.9 and 1.0. Based on the results, it can be concluded that the proposed TSPCVM would greatly reduce bus delay at signalized intersection, no matter what congestion level and conflicting condition are.

The effect on other traffic users of TSPCVM was evaluated under various congestion conditions, including near capacity volume condition. The results show that, under all circumstances, TSPCVM causes no adverse effect. Hence, TSPCVM minimizes installation and maintenance cost in the sense that it requires no need for local agencies and DOTs to perform a study of LOS and/or V/C ratio for potential TSP intersections before installation.

Future research could consider improving the TSP logic in terms of bus progression through adjacent intersections so that the delay buses saved from one intersection would not be wasted at downstream intersections. Investigation concerning the field implementation of the proposed TSP logic is also worth performing. 


\section{ACKNOWLEDGEMENT}

This research is supported by CVI/UTC at Virginia Polytechnic Institute and State University. 


\section{REFERENCES}

1. Liao, C., and G. Davis A. Simulation Study of Bus Signal Priority Strategy: Taking Advantage of Global Positioning System, Automated Vehicle Location System, and Wireless Communications. Transportation Research Record: Journal of the Transportation Research Board, No. 2034, 2007, pp. pp 82-91.

2. Al-Sahili, K., and W. Taylor. Evaluation of Bus Priority Signal Strategies in Ann Arbor, Michigan. Transportation Research Record: Journal of the Transportation Research Board, Vol. 1554, No. -1, 1996, pp. 74-79.

3. Pratt, R.,H, K. Turnbull F, J. Evans E, B. McCollom E, F. Spielberg, E. Vaca, and J. Kuzmyak Richard. Traveler Response to Transportation System Changes: Interim Handbook. TCRP Web Document, No. 12, 2000, pp. v.p.

4. Muthuswamy, S., W. McShane, and J. Daniel. Evaluation of Transit Signal Priority and Optimal Signal Timing Plans in Transit and Traffic Operations. Transportation Research Record: Journal of the Transportation Research Board, Vol. 2034, No. -1, 2007, pp. 92-102.

5. Lee, J., A. Shalaby, J. Greenough, M. Bowie, and S. Hung. Advanced Transit Signal Priority Control with Online Microsimulation-Based Transit Prediction Model. Transportation Research Record: Journal of the Transportation Research Board, No. 1925, 2005, pp. pp 185-194.

6. Hu, J., B. Park "Brian", and A. Parkany Emily. Transit Signal Priority with Connected Vehicle Technology. In , 2014, pp. 20p.

7. Balke, K.,N, C. Dudek L, and T. Urbanik. Development and Evaluation of Intelligent Bus Priority Concept. Transportation Research Record, No. 1727, 2000, pp. p. 12-19.

8. Chang, G., M. Vasudevan, and C. Su. Modeling and Evaluation of Adaptive Bus-Preemption Control with and without Automatic Vehicle Location Systems. Transportation Research Part A: Policy and Practice, Vol. 30, No. 4, 1996, pp. p. 251-268.

9. Ekeila, W., T. Sayed, and M. El Esawey. Development of a Dynamic Transit Signal Priority Strategy. In , 2009, pp. 20 p.

10. Zlatkovic, M., A. Stevanovic, and P. Martin T. Development and Evaluation of Algorithm for Resolution of Conflicting Transit Signal Priority Requests. Transportation Research Record: Journal of the Transportation Research Board, No. 2311, 2012, pp. pp 167â€"175.

11. Ma, W., K. L. Head, and Y. Feng. Integrated Optimization of Transit Priority Operation at Isolated Intersections: A Person-Capacity-Based Approach. Transportation Research Part C: Emerging Technologies, Vol. 40, No. 0, 2014, pp. 49-62.

12. Ma, W., and Y. Bai. Serve Sequence Optimization Approach for Multiple Bus Priority Requests Based on Decision Tree. In , 2008, pp. pp 605-615. 
13. He, Q., K. L. Head, and J. Ding. Heuristic Algorithm for Priority Traffic Signal Control. Transportation Research Record: Journal of the Transportation Research Board, No. 2259, 2011, pp. 1-7.

14. Liu, H. X., X. Wu, W. Ma, and H. Hu. Real-Time Queue Length Estimation for Congested Signalized Intersections. Transportation Research Part C: Emerging Technologies, Vol. 17, No. 4, 2009, pp. 412-427.

15. Rakha, H.,Ahmed, and K. Ahn. Transit Signal Priority Project - Phase II: Field and Simulation Evaluation Results. , 2006.

16. Cost/Benefit Analysis of Converting a Lane for Bus Rapid Transitâ€'Phase II Evaluation and Methodology. NCHRP Research Results Digest, No. 352, 2011, pp. 28p.

17. VDOT, T. E. D. Traffic Operations Analysis Tool Guidebook.

http://www.virginiadot.org/business/resources/traffic engineering/VDOT_Traffic_Operations_A nalysis_Tool_GuidebookV1.1-August2013.pdf, Accessed Dec 15, 2013.

18. PTV. VISSIM 5.10 User Manual., Jul. 2008.

19. PTV. VISSIM 5.10-03 COM Interface Manual., Sep. 2008.

20. Highway Capacity Manual 2010. , 2010. 


\title{
INTELLIGENT TRANSIT SIGNAL PRIORITY CONSIDERING BUS PROGRESSION
}

By

\author{
Jia $\mathrm{Hu}^{*}$,1) \\ Ph.D. Candidate
}

Tel: 919-744-9842, Email: jh8dn@virginia.edu

\section{Byungkyu "Brian" Park, Ph.D. 1)}

Associate Professor

Tel: (434) 924-6347, Email: bpark@virginia.edu

\section{Young-Jae Lee, Ph.D. ${ }^{2)}$}

Associate Professor

Tel: (443) 885-1872, Email: YoungJae.Lee@morgan.edu

6) Department of Civil and Environmental Engineering

University of Virginia

P.O. Box 400742

Charlottesville, VA 22904-4742

Fax: (434) 982-2951

1) Department of Transportation and Urban Infrastructure Studies Morgan State University

1700 E. Cold Spring Lane, Baltimore, Maryland 21251

Fax: (443) 885-8218

June 2014

Submitted for consideration for presentation at the $94^{\text {th }}$ Annual Meeting of the Transportation Research Board, January 12-16, 2015

Word Count: 5,148 text words plus 2,000 for figures/tables $(8 * 250)=7,148$ total

*Corresponding Author 


\section{ABSTRACT}

Transit Signal Priority (TSP) has been studied as a control strategy that offers preferences to transit vehicles at signalized intersections. This paper presents an enhanced TSP logic based on Connected Vehicle (CV) technology to address limitations identified in the previously developed TSPCV mechanism: Globally optimized TSP with Connected Vehicle (GTSPCV). As opposed to the previously developed TSPCV logic which optimizes bus performance on an individual intersection basis, the GTSPCV considers the coordination of the intersections and progression of the bus. Both TSP take advantage of the two-way communications and additional and more accurate information provided by Connected Vehicle technology.

The logic developed in this research is evaluated both analytical and microscopic traffic simulation approaches. Both analytical tests and simulation evaluations compared four scenarios: without TSP (NTSP), conventional TSP (CTSP), Locally optimized TSP with Connected Vehicle (LTSPCV), and Globally optimized TSP with Connected Vehicle (GTSPCV). The measures of effectiveness used include bus delay and total travel time of all travelers. The performance of GTSPCV is compared against conventional TSP (CTSP) conditions under four congestion levels and five intersection spacing cases. The results show that the GTSPCV greatly reduces bus delay at signalized intersection for all congestion and spacing levels considered. Although the LTSPCV is not as efficient as GTSPCV, it still demonstrates sizable improvement over CTSP. The sensitivity analysis on intersection spacing reveals that, as long as the intersections are not too closely located, LTSPCV can produce a delay reduction up to $30 \%$. Simulation based evaluation results show that the GTSPCV logic reduces the bus delay between $35 \%$ and $68 \%$ compared to the conventional TSP. The range of improvement corresponding to the four different $\mathrm{v} / \mathrm{c}$ ratios tested, which are $0.5,0.7,0.9$ and 1.0 , respectively. No statistically significant negative effects are observed except when $\mathrm{v} / \mathrm{c}$ ratio equals1.0. 


\section{INTRODUCTION}

Transit Signal Priority (TSP), or sometimes referred to as bus priority, is a collection of techniques that provide preference to transit buses at the signalized intersections. By adjusting the traffic signal plan according to bus arrivals, the delay that transit buses experience at intersections will be reduced. Therefore, travel time can be reduced to improve the transit service quality. The technology has been applied in many cities in Europe, Asia and North America. In the US, Seattle, Portland, Los Angeles, Chicago and several other large cities have all implemented conventional TSP systems (1).

The TSP that has been installed in all the aforementioned cities is restricted in many ways. These conventional TSP (CTSP) usually only provides simple strategies like extension to or early start of the originally planned green time. When a transit bus approaching a signalized intersection sends out a TSP request, a quick calculation is generally performed on site using a bus arrival time model based on historical data. If the bus is expected to arrive shortly before the original green time, the green time starts early; else if the bus is expected to arrive shortly after the original green time ends, the green time is extended (2). One can expect the performance of the CTSP is greatly affected by the quality of the historical data. Anyhow, because the data fed into the model is either outdated or not accurate, the bus arrival time forecast could be severely biased. The inaccurate forecast of the bus arrival time could lead to the waste of extra TSP green time and cause unnecessary adverse effects on side streets. Additionally, even with extension and/or early start, conventional TSP green time can only cover a small portion of a traffic signal cycle so that a large portion of buses may not benefit from the TSP.

A next generation TSP logic based on Connected Vehicle technology (TSPCV) has been developed to address the challenges identified in the conventional TSP (3). This new TSP takes advantage of the resources provided by Connected Vehicle (CV) technology, including two-way communications between the bus and the traffic signal controller, accurate bus location detection and prediction, and the number of passengers. The key feature of the previously developed TSP logic is green time re-allocation, which moves green time instead of adding extra green time. The TSP was also designed to be conditional. That is, delay per person served as the most important criterion deciding whether TSP is to be granted. Based on the simulation results, it was concluded that the TSPCV provided buses with more accuracy and better effectiveness. Furthermore, it accommodates a higher percentage of transit buses than conventional TSP. Its performance was compared against conventional TSP (CTSP) and no-TSP (NTSP) conditions under various congestion levels. The results based on microscopic traffic simulation show that the TSPCV greatly reduces the bus delay at signalized intersection without causing negative effects on side streets.

Although, the previously developed TSPCV logic conquers the drawback of CTSP in many ways, it was under the assumption of an isolated intersection condition. In other words, the coordination between adjacent intersections is not considered. This particular consideration is 
important because the travel time savings generated at the upstream intersection could not be maintained if the TSP bus stops at the next intersection due to lack of progression.

In terms of granting green time, conventional TSP only has two strategies that are "green extension" and "red truncation" (2). One can expect that if the background timing plan on a corridor is already coordinated, the granted TSP green progression band would be coordinated by default. Hence, no extra mechanism needs to be developed in order to maintain the progression of a CTSP bus along a coordinated corridor. As a result, only one literature is reported regarding CTSP bus progression on an uncoordinated corridor. The logic of that research is simple, which is declining TSP requests for buses that cannot make through intersections between two consecutive bus stops (4). This logic is definitely not applicable to TSPCV mechanism and will certainly not produce as much as delay savings.

On the other hand, a number of new TSP strategies have been added to the TSP logic library in order to address the drawback of conventional TSP technology. Green time extension at other phase transitions with the help of AVL (5), cycle extension during rush hours: compensation is introduced to limit the adverse effects on the side streets by cutting or skipping the time from the non-bus phase (6), and finally combining TSP consideration into adaptive signal control $(1,7)$. However, as the complication of those newly developed TSP mechanisms increases, the underlying progression of the CTSP is compromised. Unfortunately, none of these newly developed TSP mechanisms include the bus progression component to their algorithms. As a matter of fact, most TSP logic cases were evaluated under one isolated intersection condition and their performance on a corridor was not investigated.

The purpose of this research is to enhance the previously developed TSPCV logic so that the coordination between consecutive intersections is explicitly considered. There are two approaches to realize this coordination:

1. Install the previously developed TSPCV logic on both intersections. Although the progression is not $100 \%$ assured by this solution, it is possible for a sizable portion of the bus group to be granted green on both intersections and thus maintain the progression. Since this solution optimizes the timing plan on each individual intersection basis, it will be referred to as Locally Optimized TSPCV (LTSPCV).

2. Enhance the previously developed TSPCV so that it considers the coordination of the intersections and progression of the bus. Since this solution optimizes consecutive intersections as a whole, it will be referred to as Globally Optimized TSPCV (GTSPCV).

In summary, the objectives of this paper are:

1. Test the performance of the LTSPCV.

2. Develop an optimization algorithm for the GTSPCV.

3. Evaluate the newly developed GTSPCV logic.

The remainder of this paper describes the structure of the enhanced TSPCV, presents analytical and simulated test results and findings, and identifies the contributions. 


\section{LOGIC ARCHITECTURE DESCRIPTION}

The proposed GTSPCV logic is built upon the foundation of the previously developed TSPCV logic (3). Hence, several characteristics are inherited from the previous logic. Firstly, the cooperation between transit buses and traffic signal is required and enabled. When a bus approaching an intersection sends a priority request, not only the traffic controller tries to accommodate the buses, but also the bus needs to travel at a reasonable speed to increase the portion of buses that can be granted with TSP. The bus speed is recommended based on remaining/expected queue, road geometry and normal signal timing plan. Secondly, as shown in Figure 1, the TSP logic proposed is the green time reallocation, in other words, instead of adding additional green time to the original timing plan the proposed TSP logic splits the original green time and compensates part of it to when green time is mostly needed by a transit bus. This mechanism makes sure that all the TSP green time is fully used. It is either discharging remaining queue or letting go the bus. Compared to the conventional TSP, unnecessary TSP green time is reduced to the minimum.

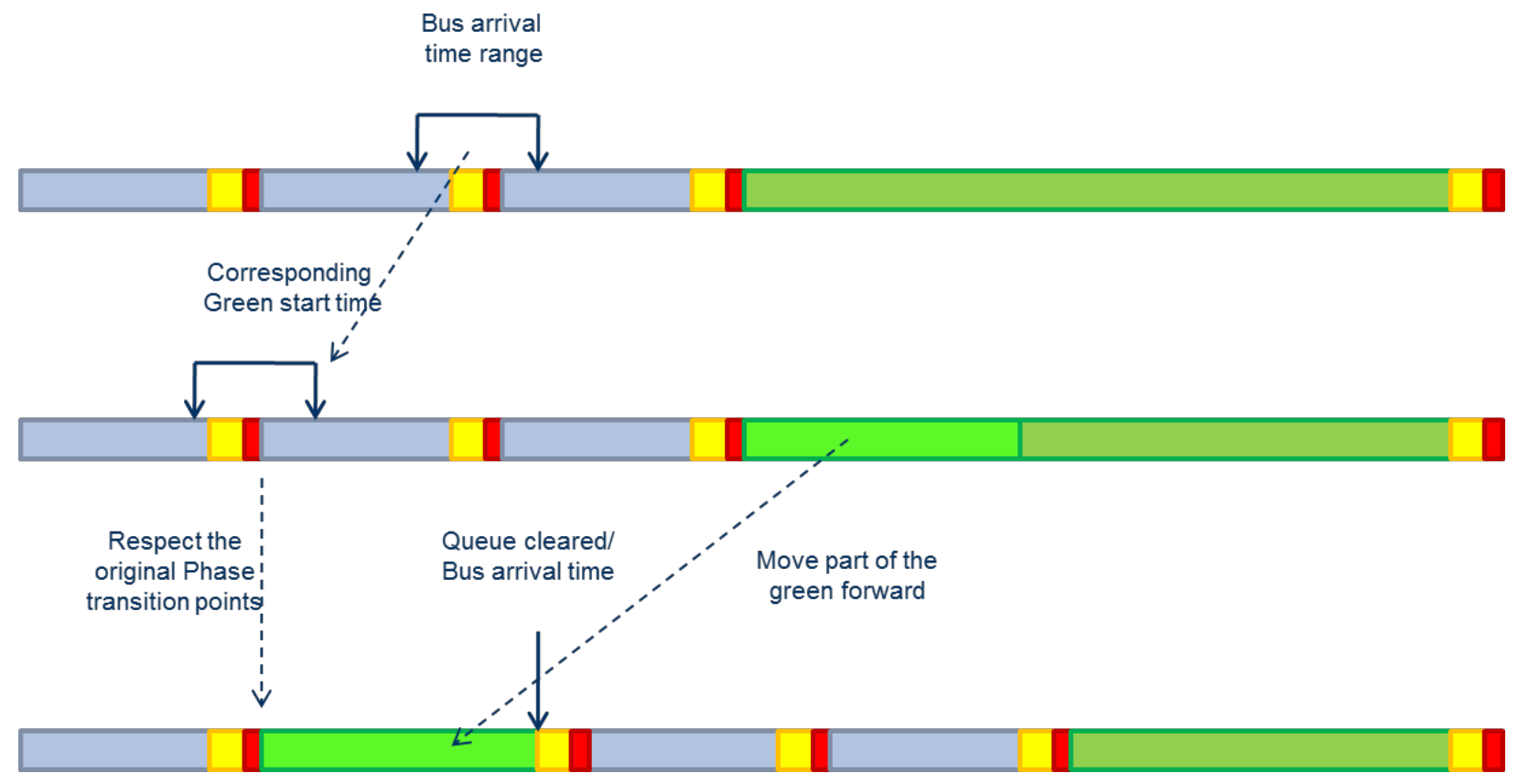

\section{FIGURE 1 Illustration of green reallocation.}

Finally, TSP green time is granted conditionally based on two criteria which are schedule adherence and delay per person. Connected Vehicle (CV) technology is required to enable twoway communications between the bus and the traffic signal control and provide information, including accurate bus location detection and prediction, and the number of passengers. Apart from the aforementioned characteristics, the proposed GTSPCV optimizes TSP timing plan 


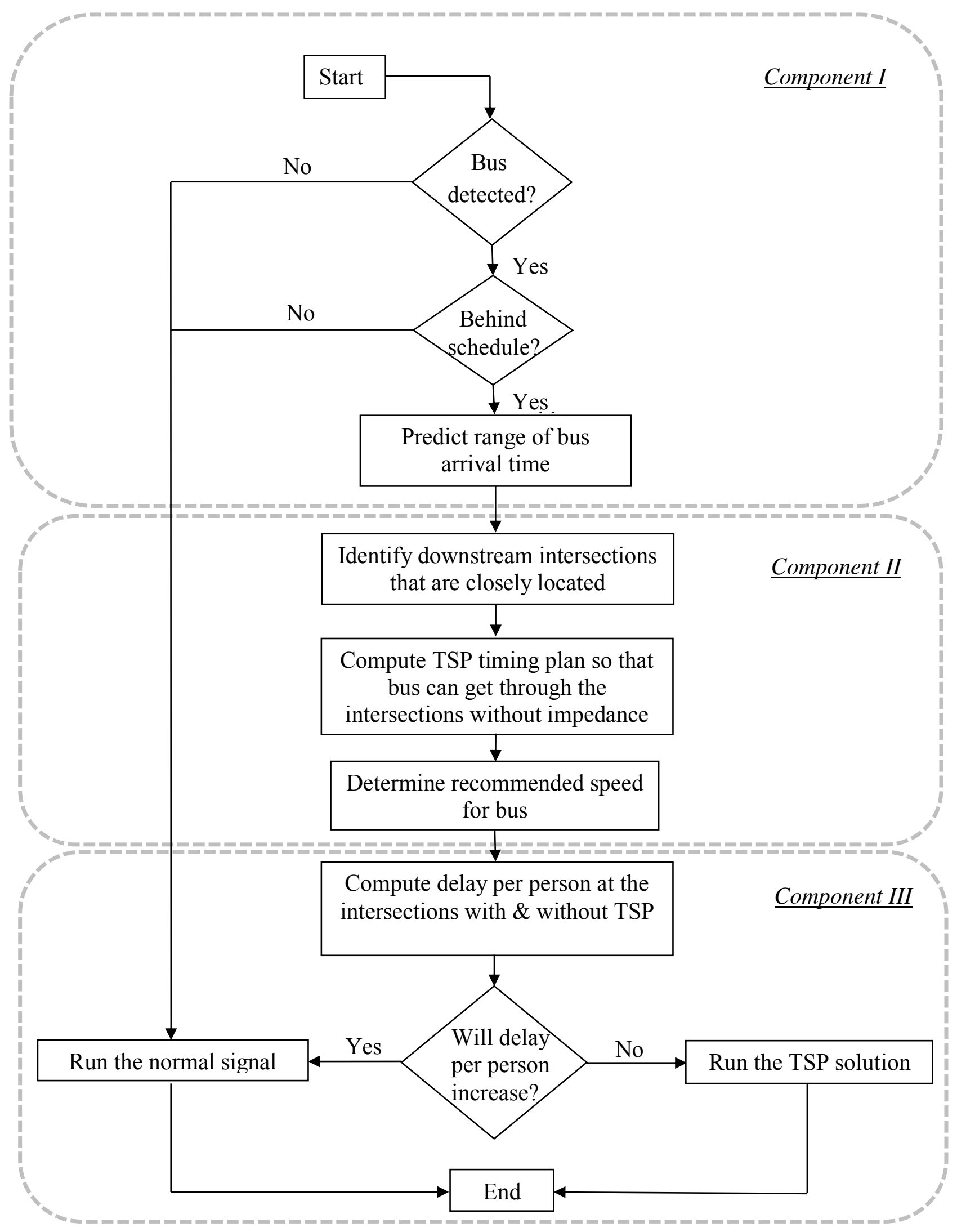

Figure 2 The Structure of GTSPCV 
globally across all intersections along the corridor of interest and considers the progression of the bus. Thereafter, the TSP will only be granted unless the bus could travel through the corridor without impedance and the new timing plan causes no adverse effect to other traffic users at the same time. Figure 2 displays the architecture of the GTSPCV. The logic is composed of three major components:

1. Arrival time prediction component, in which the bus arrival time range at the subject intersection is predicted.

2. TSP timing plan and bus speed calculation component, in which, given the arrival time range, the algorithm generates a timing plan that will have minimum impact on general traffic users, and calculates the corresponding recommended bus speed.

3. Logic assessment and implementation component, in which the TSP timing plan will be compared against the normal signal time (winner overwrites the other) and the recommended bus speed will be transmitted to the coming bus.

Detailed information regarding the three components of LTSPCV is provided in the literature (3). The following presents the logic of GTSPCV.

\section{Arrival Time Prediction Component}

One of the great advantages of integrating TSP into a CV system is the two-way communications between the roadside equipment (RSE) and traffic users which in this case is the bus. A CVequipped bus communicates with traffic signal controller and is capable of receiving speed instructions. Assuming the desired speed of an approaching bus could vary between $10 \%$ above and $20 \%$ below the speed limit, then the prediction result generated from this component is not a fixed number but a time range. The range of arrival time is computed for the next step so that the bus can adjust its travel at various speeds to cooperate with TSP strategy.

\section{TSP Timing Plan and Bus Speed Calculation Component}

The first step of generating an optimum timing plan is to compute a range of TSP green start time using the arrival time range acquired from the previous component. The TSP green start time is calculated based on the goal that the TSP green is inserted exactly when it is needed and for the duration it is needed. The duration of the TSP green time is determined so that the queue is cleared before the bus arrives at the intersection. In other words, the bus will catch up with the end of the queue right at the stop bar of the intersection. The calculation of the adopted real-time queue length estimation is based on the model developed by Liu (8) which is an extension of the shock wave theory. Vehicle arrival rates at the intersections follow the platoon dispersion model (9). In other words, when the upstream intersection is discharging the queue $\left(t<t_{q}\right)$, then,

$$
Q_{t}=s\left(1-(1-F)^{t}\right),
$$

After the queue at the upstream intersection is fully discharged, $\left(t>t_{q}\right)$, then,

$$
Q_{t}=s\left(1-(1-F)^{t_{q}}\right) \times(1-F)^{t-t_{q}},
$$


Where $Q_{t}$ is the number of vehicles arrive at time $t ; s$ is the queue discharging rate at the upstream intersection; $t_{q}$ is the queue dissipating time at the upstream intersection; $F$ is a parameter that represents the rate that a platoon disperses over time and space.

It is noted that the cycle length will be the same even when the TSP green is inserted, because the TSP green time is spliced from the original green time of the direction of the bus. So strictly speaking, the extra TSP green time is "moved" rather than "inserted" or "added." The inserted green time taken from the certain direction is $100 \%$ used to clear the traffic for that direction. Therefore, theoretically speaking, not a single second is wasted during the TSP.

By calling the previous component, a range of bus arrival time is first computed for the most upstream intersection. Using this time range, the bus arrival time range for the sequential intersection can be calculated. This same calculation process is applied on all the downstream intersections. In the end, the predicted bus arrival time is generated as a pack of time ranges. The number of ranges represents the number of intersections considered. Each time stamp with these time ranges represents the time the bus leaves that particular intersection. It is also the end of the TSP green time at that intersection. Based on the mechanism described in previous paragraphs, this component then finds a corresponding set of ranges of TSP green end time. While there will be numerous TSP timing plans depending on when the bus arrives, the potential TSP timing plans are first narrowed down using the following rules:

1. A TSP green time is preferred to start at the end of phase than to cut into the middle of a non-TSP signal phase (for better safety and drivers' expectation)

2. If a TSP green has to start in the middle of a phase, it is preferred that the bus travels at its normal speed

3. Minimum green time is required for both the TSP green time and the original timing plan.

4. The bus is able to pass all the intersections without impedance. In case bus cannot receive green (either TSP green or original green) at all the intersections, the TSP time plan is not implemented.

Based on these rules, the algorithm then finds a pool of preferred TSP start and end times from the time range that TSP can possibly start and terminate, and then it feeds them into an optimization algorithm. This optimization algorithm is activated every time a TSP request is received, and it does an exhaustion search for all the possible TSP start and end time and calculates total person delay at all the intersections for a predetermined duration of time. The TSP timing plan with the least total person delay is select as the candidate for the next step. The objective function estimating total person delay can be expressed as follows:

The choice variables include: $T_{T S P e n d 1}, T_{T S P e n d 2} \ldots T_{T S P e n d k}$.

$$
\text { Minimize } \sum_{K=1}^{k}\left(\sum_{c y c l e=1}^{c y c l e=3} \int_{0}^{T} D_{i} * O c c_{i}+\sum_{c y c l e=1}^{c y c l e=3} \int_{0}^{T} D_{b u s} * O c c_{b u s}\right) \text {. }
$$


Subject to:

$$
\begin{aligned}
& \sum_{j}\left(G_{j_{-} b e f o r e_{-} k}+G_{j_{-} a f t e r_{-} k}\right)+G_{T S P_{-} k}+G_{\text {remain } \_k}=T=\text { constant } \\
& T_{T S P e n d \_k}-T_{T S P s_{-} k}=G_{T S P \_k} \\
& T_{\text {BAlow_k }} \leq T_{\text {TSPend_k }} \leq T_{\text {BAup_k }} \\
& T_{\text {TSPs_k }}=\sum_{j} G_{j \_b e f o r e \_} \\
& G_{j_{-} b e f o r e \_k}+G_{j_{-} a f t e r_{-} k}=\text { Constant } \\
& G_{j_{-} b e f o r e \_k} \geq G_{\min } \text { or } G_{j_{\_} \text {before } k}=0 \\
& G_{j_{-} a f t e r_{-} k} \geq G_{\min } \text { or } G_{j_{-} a f t e r_{-} k}=0 \\
& G_{T S P_{-} k} \geq G_{\min } \text { or } G_{T S P_{-} k}=0 \\
& G_{\text {remain_k }} \geq G_{\min } \text { or } G_{\text {remain_k }}=0
\end{aligned}
$$

Where:

$k$ : The number of intersections identified that are closely located with each other.

$T$ : cycle length at the intersection

$D i$ : Delay of vehicle i

$D_{\text {bus }}$ : Delay of bus

$O c c_{i}$ : Occupancy on vehicle $i$

Occ $_{\text {bus }}$ : Occupancy on the bus

$G_{j \_ \text {before } \_}$: Green time for phase $\mathrm{j}(1,2$, or 3$)$ before TSP green at the intersection $k$

$G_{j_{-} a f t e r} k$ : Green time for phase $\mathrm{j}(1,2$, or 3$)$ after TSP green at the intersection $\mathrm{k}$

$G_{T S P_{-} k}$ : TSP green time at the intersection $\mathrm{k}$

$G_{\text {remain } \_ \text {: }}$ Remaining green time for lane group with bus after taking out the TSP green at the intersection $\mathrm{k}$

$G_{m i n}$ : minimum green time requirement

$T_{T S P e n d} k$ : End time of TSP green at the intersection $\mathrm{k}$

$T_{T S P S_{-} k}:$ Start time of TSP green at the intersection $\mathrm{k}$

$T_{B A l o w \_}$: Lower bound of bus arrival time range at the intersection $\mathrm{k}$

$T_{\text {BAup_k }}$ : Upper bound of bus arrival time range at the intersection $\mathrm{k}$

Once the timing plan is generated, the recommended bus speed could then be computed so that the bus would travel through the intersection right after the queue ahead is cleared and before the TSP green phase ends.

\section{Logic Assessment and Implementation Component}

After a TSP timing plan is determined, the algorithm will compare the "with TSP" scenario against the "normal timing" scenario. Since the number of passengers on board is likely to be 
known under the $\mathrm{CV}$ environment, the person delay performance measure is to be used. The person delay is calculated for a predefined duration of time starting from the TSP implemented cycle. In this study, a TSP timing plan will be only implemented when its corresponding person delay is less than the "no TSP" scenario.

During implementation, two major steps are conducted. First, an instruction is given to a bus about the desired recommended speed. Second, a buffer green time is possibly given to a bus in case a bus is not expected to make it through the intersection. The TSP green time would be extended up to 5 seconds to accommodate the random delay.

\section{EVALUATIONS}

\section{Study Site}

A study site with two consecutive intersections on Route 50 in Fairfax, Virginia, was selected for evaluating the proposed logic. The intersections, as presented in Figure 3, are the joints of Route 50 with Sullyfield Cir and Centreville. The site was chosen because the signal timing is coordinated and has been calibrated shortly before the volume data was collected (10).

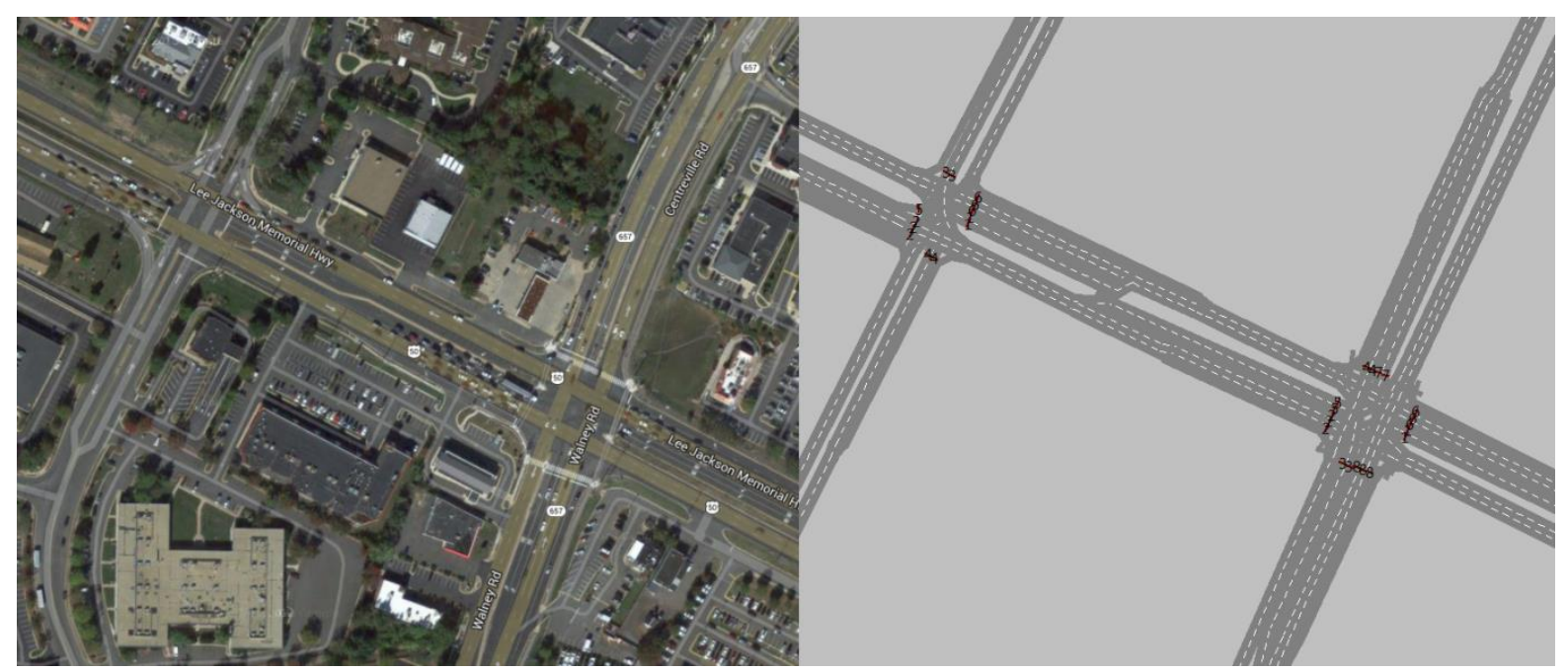

\section{FIGURE 3 Study site.}

\section{Methodology}

Both analytical tests and simulation evaluations have been performed for the proposed GTSPCV as well as LTSPCV, conventional TSP and no TSP cases. As noted, the test network is a calibrated VISSIM model of the Route 50 in Fairfax, Virginia, as shown in FIGURE . Vehicle volumes and turning movements are actual peak-hour data collected from the site. Details concerning the calibration of the model and data collection process are provided in the literature (10). To avoid multiple buses, while it is not likely, it is assumed that a bus is traveling EB on Route 50 with a mid-block bus stop located 750 feet upstream of the first intersection. The speed limit on Route 50 is $45 \mathrm{mph}$, therefore, buses are allowed to travel within the speed range between $35 \mathrm{mph}$ and $50 \mathrm{mph}$ (i.e., between $20 \%$ below and $10 \%$ above speed limit). The TSP logic is activated when buses pass 0.5 mile upstream of the first intersection. As aforementioned, 
a duration of time needs to be predefined for person delay calculation. In this case study, a duration of 3 signal cycle is adopted. It is noted that the three cycles are used to be long enough to capture residual effects caused by TSP and be short enough to prevent including another TSP request, given three cycles of 120 -second cycle is about the minimum bus headway. Given the size of the problem, the optimization method used is the simple exhaustive search. When the number of intersections considered increases, more advanced optimization method may be considered. For example, the Damped Newton model, Levenbery-Marquardt method, etc. However, at this moment, the exhaustive search method can already solve the problem within one tenth of a second. Two assumptions are made for the buses: 1, bus occupancy is 40; 2, dwell time at bus stops is 30 seconds with 2 seconds standard deviation. The values are adopted from an NCHRP research regarding bus rapid transit (11). Consider that the CV technology is capable of providing accurate dwell time prediction; the variation is set to be moderately low.

Both analytical tests and simulation evaluations compared four scenarios: without TSP (NTSP), conventional TSP (CTSP), Locally optimized TSP with Connected Vehicle (LTSPCV), and Globally optimized TSP with Connected Vehicle (GTSPCV). The detailed about the analytical tests and simulation evaluations will be presented in the following sections. The conventional TSP logic compared here is TSP with AVL and an on board passenger counting system. In other words, CTSP uses the state-of-the-art TSP plus a more accurate bus arrival time forecast module. The difference between CTSP and TSPCV is that the logic CTSP utilizes is a simple one (green extension only) with no cooperative interactions between the bus and the traffic signal controller. The CTSP will grant 10 -second extra green time to buses which arrive within 10 seconds of the end of normal green time. In case the bus could not make through the intersection within that 10 seconds, CTSP will add to the previous 10 seconds with up to 5 seconds to accommodate the late arrival. The logic follows the real implementation in the Northern Virginia (12). The difference between two approaches of TSPCV is that the GTSPCV considers the coordination of the intersections and progression of the bus while LTSPCV does not.

The measures of effectiveness used are bus delay and total travel time of all travelers. Bus delay quantifies the effectiveness of various TSP treatments while the total travel time demonstrates whether the adverse effect is caused.

To consider the effect of simulation randomness, 20 simulation runs were performed for each scenario and the MOEs for each scenario were averaged from the output of each of the 20 runs. Minimum sample size requirement was checked to make sure that sufficient number of simulation runs was achieved to represent the entire population. Minimum sample size was calculated using the formula recommended by the Virginia Department of Transportation (13), which is:

$$
\mathrm{N}=Z^{2} * \frac{S_{S}^{2}}{\left(X_{S} * E\right)^{2}},
$$


where,

$Z$ : Number of standard deviations away from the mean corresponding to the required confidence level in a normal distribution. In this study, confidence level is set to be $95 \%$.

Ss: Sample standard deviation.

$X s$ : Sample mean.

$E$ : Tolerable error. In this study, $\mathrm{E}=10 \%$.

Finally, all the differences have been checked for statistical significance. The purpose is to ensure that all the improvements or adverse effects claimed in the result session are statistically significant. Paired two tailed T-test was utilized, since data in comparison was collected from the same site, and the confidence level tested was $95 \%$.

\section{Analytical Test}

The analytical test is a deterministic calculation that quantifies the performance of the proposed TSP logic on the theoretical level. Here are all the factors considered. Volume is the average flow rate collected from the study site during peak hour. Signal timing plan is also adopted from the current timing plan in the field. Saturation flow rate is the default value adopted from the Synchro which is $1900 \mathrm{veh} / \mathrm{h} / \mathrm{ln}$. Queue length at the stop bar is estimated based on the constant arrival rate assumption. All possible TSP activation scenarios are considered. The cycle length at the intersection is 120 seconds. Assuming a TSP can be activated at any given second, there are 120 possible situations. The stop delay for bus is calculated by averaging these 120 situations. All three treatments have been computed and compared to NTSP condition. Field peak-hour volume data is applied, which is near capacity situation. The delay savings are summarized in Table 1.

TABLE 1 Bus Delay Reduction under Various Intersection Spacing

\begin{tabular}{ccccccc}
\hline & $\begin{array}{c}\text { Distance } \\
\text { (Mile) }\end{array}$ & $\mathbf{0 . 1 4}$ & $\mathbf{0 . 2 4}$ & $\mathbf{0 . 3 4}$ & $\mathbf{0 . 4 4}$ & $\mathbf{0 . 5 4}$ \\
\hline delay (Sec) & LTSPCV & 33.3 & 30.4 & 30.0 & 26.6 & 25.4 \\
& GTSPCV & 18.8 & 21.8 & 13.7 & 15.2 & 14.0 \\
& CTSP & 33.4 & 33.4 & 33.4 & 33.4 & 33.4 \\
\hline \multirow{5}{*}{ Improvements } & NTSP & 36.4 & 36.4 & 36.4 & 36.4 & 36.4 \\
& LTSPCV & $8.6 \%$ & $16.4 \%$ & $17.6 \%$ & $26.8 \%$ & $30.1 \%$ \\
& GTSPCV & $48.3 \%$ & $40.0 \%$ & $62.5 \%$ & $58.3 \%$ & $61.7 \%$ \\
& CTSP & $8.3 \%$ & $8.3 \%$ & $8.3 \%$ & $8.3 \%$ & $8.3 \%$ \\
\hline
\end{tabular}

The current spacing between the two intersections is 0.14 miles. Under such condition, LTSPCV presents similar improvement as CTSP with a reduction around 8\%, while GTSPCV demonstrates much greater benefit of $48 \%$ delay reduction. It is intuitive since the small spacing of the two intersections significantly reduces the flexibility of the LTSPCV and thus the portion of buses that is able to receive LTSPCV from both intersections largely decreases. However, one can expect that, as the spacing increases, the flexibility of LTSPCV will also increase and bring up the associated bus delay reduction.

Therefore, the research took one step ahead and performed a sensitivity analysis on the intersection spacing based on deterministic computation. To better demonstrate the results, 
TABLE is interpreted into Figure 4. The results are intuitive to show that the performance of CTSP is not affected by intersection spacing, since a fixed proportion of buses receive CTSP treatment. The benefit of LTSPCV is positively correlated with the intersection spacing. Although LTSPCV shows similar benefit as CTSP under the 0.14-mile-spacing condition, its advantage over CTSP becomes more obvious as the spacing increases over 0.24 miles. When spacing reaches 0.54 miles, the delay reduction increases to a sizable improvement of $30 \%$ which is almost 4 times the benefit of CTSP. Interestingly, GTSPCV always demonstrates superior improvement than the other two treatments, no matter what size of the spacing is. The benefit of GTSPCV grows slightly with the intersection spacing, but it does not always necessarily maintain a positive correlation. Detailed investigation reveals that the constraint of minimum green time is a major influencing factor. Despite the fact that the increase of spacing raises the flexibility on the transit buses, it also changes the pairing of timing plans from two intersections. As a result, to fulfill the minimum green time requirement, some previous feasible coordination plans become unfeasible. This is what leads to the decrease in benefit when intersection spacing increases.

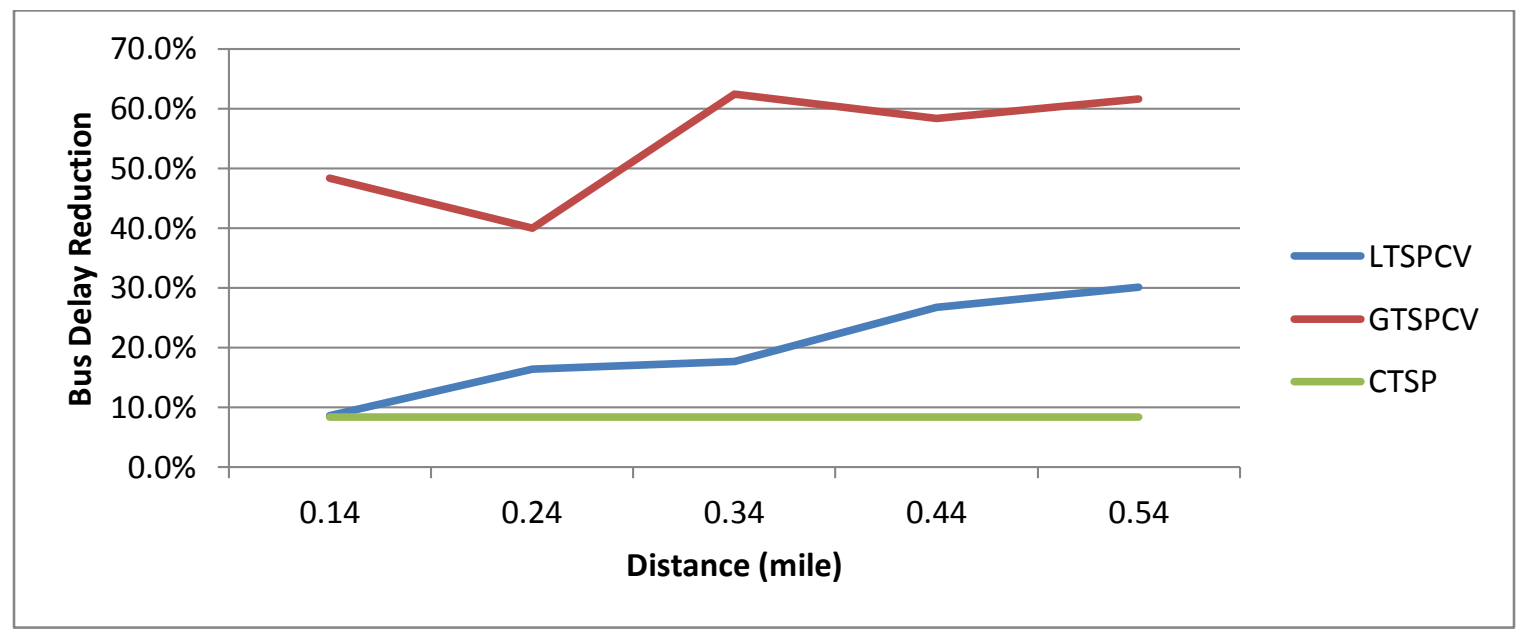

FIGURE 4 Bus delay reduction under various intersection spacing.

\section{Simulation-based Evaluation in VISSIM}

While the analytical test results show significant benefits under the proposed TSPCV logic, it does not consider any variability due to vehicle interactions and inter-arrival times. A microscopic traffic simulator can assess the performance under more plausible conditions. The microscopic simulation software package VISSIM (14) is used to evaluate the proposed TSP logic under a CV environment. A COM interface is used to assess information that would be available within a CV environment (15). The evaluation is performed under the assumption that only transit buses are connected to the traffic signal controller and other traffic users do not have $\mathrm{CV}$ equipments. In other words, $0 \% \mathrm{CV}$ market penetration except for buses. The end of queue is estimated based on incoming vehicles and outgoing vehicles at the intersection. Detailed algorithm can be found in the model developed by Liu (8) which is an extension of the shock wave theory. The input to this algorithm is the average flow rates from all travel directions. Therefore, the data extracted via COM interface would include speed and position of bus, 
number of passengers on board, number of potential passengers at the bus stop, number of vehicles passing the intersection and volume from all four approaches. Besides, the COM interface is used to change the signal timing plan during the simulation. All programs are coded in Microsoft EXCEL VBA.

As noted, the test network is a calibrated model of 2 consecutive intersections on Route 50 in Fairfax, Virginia. Vehicle volumes and turning movements are actual peak-hour data collected from the site. Bus dwelling time at the stop is 30 seconds average with a standard deviation of 2 seconds. A transit bus is designed to arrive every 375 seconds. Given the cycle length is 120 seconds at the intersection; the interval of bus arrival is exactly 3 cycles plus 15 seconds. This research purposefully designed the offset to be 15 seconds so that buses within one single simulation run will arrive at different times relative to signal cycles; hence the simulation results would be less biased.

The simulation based evaluations also compared four scenarios: without TSP (NTSP), conventional TSP (CTSP), Locally optimized TSP with Connected Vehicle (LTSPCV), and Globally optimized TSP with Connected Vehicle (GTSPCV). Each scenario ran 20 times with different random seeds. It is ensured that the results show statistical significance with a $95 \%$ confidence level and 5\% tolerance error. The sample size sufficiency is assessed using the formula recommended by the Virginia Department of Transportation (13). Twenty runs are sufficient in this case.

The results from the simulation based evaluation are shown in Table 2 . The bus delay and total travel time of all vehicles were summarized and averaged from 20 simulation runs. All three TSP treatments were compared with NTSP condition and T-test was performed to validate the differences from a statistical perspective. In summary, the simulation based results support the findings from the analytical analysis. As two intersections are closely located ( 0.14 miles), LTSPCV and CTSP showed minor improvement over NTSP condition while GTSPCV significantly saved bus delay by $37 \%$. Since the delay savings generated by LTSPCV and CTSP are not statistically significant, only GTSPCV presented benefit for bus travelling through closely located intersections under near capacity volume.

The research also collected travel time data for all traffic users which is included in Table 2 as well. It is discovered that GTSPCV and LTSPCV caused a minor adverse effect on other traffic users. It is because the delay estimation module embedded in the GTSPCV logic could not accurately predict the effect of the queue-spill-back effect, and the peak-hour data collected from the field is around the capacity. Hence, some TSP requests are granted regardless of the fact that extra delay would be caused. However, the effect is minimal (less than $1 \%$ increase in travel time). When translated into delay increase, GTSPCV caused about 1 second delay per person. 
TABLE 2 Simulation Based Assessment on Various TSP Treatments

\begin{tabular}{ccccccccc}
\hline & $\begin{array}{c}\text { Bus } \\
\text { delay } \\
(\text { Sec) }\end{array}$ & $\begin{array}{c}\text { \% } \\
\text { saving }\end{array}$ & Std_Dev & T-test & $\begin{array}{c}\text { Total TT } \\
\text { (h) }\end{array}$ & $\begin{array}{c}\text { \% } \\
\text { saving }\end{array}$ & Std_Dev & T-test \\
\hline GTSPCV & 42.4 & $37.1 \%$ & 4.5 & $6.7 \mathrm{E}-08$ & 256.9 & $-0.6 \%$ & 2.9 & $6.8 \mathrm{E}-07$ \\
LTSPCV & 55.9 & $4.0 \%$ & 7.2 & $3.4 \mathrm{E}-01$ & 256.8 & $-0.5 \%$ & 3.3 & $1.1 \mathrm{E}-05$ \\
CTSP & 57.4 & $1.4 \%$ & 7.8 & $2.9 \mathrm{E}-01$ & 254.6 & $0.3 \%$ & 2.8 & $1.8 \mathrm{E}-01$ \\
NTSP & 58.1 & $0.0 \%$ & 8.3 & N/A & 255.4 & $0.0 \%$ & 2.8 & N/A \\
\hline
\end{tabular}

\section{Sensitivity Analysis on Congestion Levels}

In order to verify that the findings from the experiment are consistent with various congestion levels, a sensitivity analysis is conducted. Because LTSPCV cannot perform well under such close intersection spacing, the sensitivity study on congestion levels is not conducted for LTSPCV. Since the field collected volume data is at $\mathrm{v} / \mathrm{c}$ ratio of 1.0 , three other scenarios are tested: $\mathrm{v} / \mathrm{c}=0.5, \mathrm{v} / \mathrm{c}=0.7$ and $\mathrm{v} / \mathrm{c}=0.9$. The results have been presented in the Table 3 .

TABLE 3 Sensitivity Analysis Concerning Congestion Levels

\begin{tabular}{cccccc}
\hline v/c ratio & MOE & CTSP & GTSP & Difference & \% change \\
\hline $\mathbf{0 . 5}$ & Bus Delay & 44.1 & 28.0 & -16.1 & $-57.4 \%$ \\
& Total TT (h) & 122.4 & 122.2 & -0.2 & $-0.2 \%$ \\
$\mathbf{0 . 7}$ & Bus Delay & 47.8 & 28.5 & -19.3 & $-67.6 \%$ \\
& Total TT (h) & 171.1 & 171.6 & 0.5 & $0.3 \%$ \\
$\mathbf{0 . 9}$ & Bus Delay & 52.5 & 34.3 & -18.2 & $-53.1 \%$ \\
& Total TT (h) & 223.5 & 223.7 & 0.3 & $0.1 \%$ \\
$\mathbf{1}$ & Bus Delay & 57.4 & 42.4 & -15.0 & $-35.3 \%$ \\
& Total TT (h) & 253.6 & 256.9 & 3.3 & $1.3 \%$ \\
\hline
\end{tabular}

As noted, delay per person at the intersection is a measure that reflects adverse effects caused by TSP. When examining the results, both CTSP and GTSPCV did not cause additional person delay at various $\mathrm{v} / \mathrm{c}$ ratios except when $\mathrm{v} / \mathrm{c}=1.0$. But it is consistent with the previous results that during high volume condition ( $\mathrm{v} / \mathrm{c}=1.0)$, GTSPCV shows minor adverse effect on other traffic users. However, the results also reveal that, when volume decreases below capacity, no statistically significant increase in delay was caused by GTSPCV. On the other hand, significant reduction on the bus delay is generated by applying GTSPCV. To better represent the results, Table 3 is visualized into the Figure 5. 


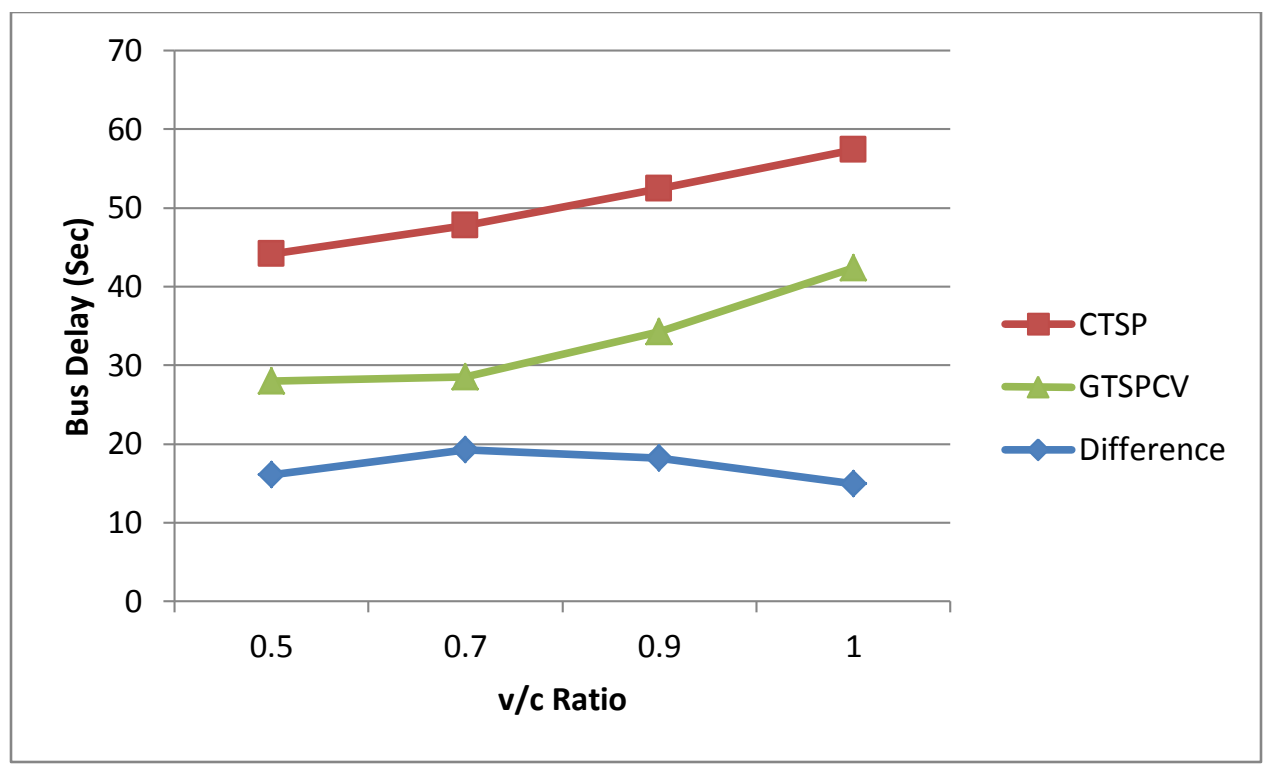

\section{FIGURE 5 Bus delay change with various congestion levels.}

As demonstrated in FIGURE, when the congestion level is low, GTSPCV would help reduce bus delays up to about $68 \%$ compared to CTSP under VISSIM simulations. As the congestion level rises, the benefit of GTSPCV decreases, while no extra delay is caused. This is because the algorithm is designed to be conditional on the person delay. When the volume becomes closer to the capacity, less portion of the green time will be granted to TSPCV to prevent TSP from causing extra delay on other travelers. As a result, the benefit would drop correspondingly, while adverse effects on side streets would still be kept under a certain level. The only exception happens when $\mathrm{v} / \mathrm{c}$ ratio rises from 0.5 to 0.7 . The reason is similar that the increase in volume leads to change in queue dissipation time which is eventually bounded by the minimum green time requirement. Hence, it is likely that more buses have been granted with GTSPCV under $0.7 \mathrm{v} / \mathrm{c}$ ratio than $0.5 \mathrm{v} / \mathrm{c}$ ratio.

\section{CONCLUSIONS AND FUTURE RESEARCH}

To address limitations in the previously developed TSPCV mechanism, an enhanced TSP logic based on Connected Vehicle technology, GTSPCV, is proposed and evaluated. The research provides a method to secure the mobility benefit generated by the intelligent TSP logic along a corridor so that the bus delay saved at an upstream intersection is not wasted at the downstream intersections. The LTSPCV logic simply applies the previously developed TSPCV mechanism on each individual intersection, while the GTSPCV considers the coordination of the intersections and progression of the bus. Both TSP logic cases take advantage of the two-way communications and additional and more accurate information provided by Connected Vehicle technology. Based on the simulation results, it can be concluded that the proposed GTSPCV provides buses with more delay savings. Furthermore, its performance is compared against conventional TSP (CTSP) conditions under various congestion levels and various intersection spacing. The results show that the GTSPCV would greatly reduce the bus delay at signalized intersections for all congestion levels and spacing considered in this paper. Although the LTSPCV is not as efficient as GTSPCV, it still demonstrated sizable improvement over CTSP. Sensitivity analysis on intersection spacing reveals that, as long as the intersections are not too 
closely located, LTSPCV can produce a delay reduction up to 30 percent. In addition, LTSPCV is superior to GTSPCV in terms of the computation burden. Therefore, GTSPCV is suitable for small networks and produce much more significant benefit, while LTSPCV generates sizable but less benefit and it is capable of handling much larger networks.

The effect on other traffic users of GTSPCV was evaluated under various congestion conditions, including near capacity volume condition. The results show that, for congestion levels below capacity, GTSPCV causes no adverse effect. Although little adverse effects on side streets are expected when the volume reaches capacity, the delay increase is minor and less than 1 second per person. Hence, it might not be a must for local agencies and DOTs to perform a study of LOS and/or V/C ratio for potential TSP intersections before installing GTSPCV.

This research evaluated the performance of the proposed TSP logic under the $0 \%$ market penetration condition. The purpose is to show the least improvement. Future research could consider investigating the improvement of GTSPCV generated with high CV technology coverage. In addition, the next phase of this research could proceed to consider multiple buses and multiple corridors, in other words, a TSP logic that accommodates conflicting TSP requests on a transportation network. This ultimate TSP logic can be embedded with the city traffic control system and would be able to optimize the transit system performance on an area basis. This research adopted person delay as one of the condition criteria, other measurements can be tested in future research, for example, fuel consumption, emissions, etc. 


\section{REFERENCES}

1. Liao, C., and G. Davis A. Simulation Study of Bus Signal Priority Strategy: Taking Advantage of Global Positioning System, Automated Vehicle Location System, and Wireless Communications. Transportation Research Record: Journal of the Transportation Research Board, No. 2034, 2007, pp. pp 82-91.

2. Lee, J., A. Shalaby, J. Greenough, M. Bowie, and S. Hung. Advanced Transit Signal Priority Control with Online Microsimulation-Based Transit Prediction Model. Transportation Research Record: Journal of the Transportation Research Board, No. 1925, 2005, pp. pp 185-194.

3. Hu, J., B. Park "Brian", and A. Parkany Emily. Transit Signal Priority with Connected Vehicle Technology. In, 2014, pp. 20p.

4. Ma, W., W. Ni, L. Head, and J. Zhao. Effective Coordinated Optimization Model for Transit Priority Control Under Arterial Progression. Transportation Research Record: Journal of the Transportation Research Board, No. 2356, 2013, pp. pp 71â€“"83.

5. Balke, K.,N, C. Dudek L, and T. Urbanik. Development and Evaluation of Intelligent Bus Priority Concept. Transportation Research Record, No. 1727, 2000, pp. p. 12-19.

6. Ekeila, W., T. Sayed, and M. El Esawey. Development of a Dynamic Transit Signal Priority Strategy. In , 2009, pp. 20 p.

7. Chang, G., M. Vasudevan, and C. Su. Modeling and Evaluation of Adaptive Bus-Preemption Control with and without Automatic Vehicle Location Systems. Transportation Research Part A: Policy and Practice, Vol. 30, No. 4, 1996, pp. p. 251-268.

8. Liu, H. X., X. Wu, W. Ma, and H. Hu. Real-Time Queue Length Estimation for Congested Signalized Intersections. Transportation Research Part C: Emerging Technologies, Vol. 17, No. 4, 2009, pp. 412-427.

9. Mathew, J., H. Thomas, A. Sharma, L. Devi, and L. Rilett. Studying Platoon Dispersion Characteristics Under Heterogeneous Traffic in India. Procedia - Social and Behavioral Sciences, Vol. 104, No. 0, 2013, pp. 422-429.

10. PARK, B., and J. Schneeberger D. Microscopic Simulation Model Calibration and Validation: Case Study of VISSIM Simulation Model for a Coordinated Actuated Signal System.

Transportation Research Record, No. 1856, 2003, pp. p. 185-192.

11. Cost/Benefit Analysis of Converting a Lane for Bus Rapid Transitâ€'Phase II Evaluation and Methodology. NCHRP Research Results Digest, No. 352, 2011, pp. 28p.

12. Rakha, H.,Ahmed, and K. Ahn. Transit Signal Priority Project - Phase II: Field and Simulation Evaluation Results. , 2006. 
13. VDOT, T. E. D. Traffic Operations Analysis Tool Guidebook.

http://www.virginiadot.org/business/resources/traffic_engineering/VDOT_Traffic_Operations_A nalysis_Tool_GuidebookV1.1-August2013.pdf, Accessed Dec 15, 2013.

14. PTV. VISSIM 5.10 User Manual., Jul. 2008.

15. PTV. VISSIM 5.10-03 COM Interface Manual., Sep. 2008. 


\section{IMPLEMENTATION PLAN}

It is valuable to test the feasibility of the developed TSPCV system in the field, as it is one of the few applications that are capable of being implemented in the early stage of connected vehicle technology roll-out. This document contains detailed information regarding the design of possible field implementation in the Northern Virginia area. The document is written under the assumption that DOTs and bus agencies provide permission to install devices onto buses and make changes to signal controls.

As stated in the previous papers, the developed TSPCV logic has been evaluated successfully using a microscopic simulation. The logic will then be tested at the Smart Road to ensure the connected vehicle technology harnessed TSP logic performs as expected on the functional level. After successful testing on the Smart Road in Blacksburg, VA, the researcher proposes to implement a real world testing in the Northern Virginia area. The purpose is to confirm the mechanism developed works under real world condition. The mechanism should work in terms of both saving bus travel time and minimize adverse effect on other traffic users.

A study site needs to be selected. In order to not interrupt too much on the general traffic, it is suggested that a two-consecutive-intersection segment shall be chosen as the test bed. Since the simulation study picked the joints of Route 50 with Sullyfield Cir and Centreville, the same site is preferred.

\section{DATA}

Two bus routes should be selected for the experiment. One route travels on the major street and the other passes through the minor street. This fashion of selecting bus routes enables possible perpendicular TSP conflict and three-TSP-request conflict. The fleet should be installed with data collection devices. The details regarding the devices are provided in the following "devices" section. Depending on the budget, at least two weeks' worth of data should be collected. One week for without TSPCV condition and the other week for with TSPCV condition. This way of collecting data enables time-of-day and day-of-week comparison so that the before and after conditions can be compared under similar traffic conditions.

Here are the measurement of effectiveness (MOE) recommended:

Total travel time: The total travel time of all vehicles passing through the study site should be collected. It is the MOE that quantifies the adverse effect caused by TSP. Bluetooth data could serve as the data source.

Bus travel time: Bus travel time passing through the study site should be collected. It is the MOE that quantifies the benefit of the TSP. It could be computed using the bus GPS data collected. 
Success rate: It is percentage that TSP is successfully implemented. In other words, the percentage of buses that make through the study site with the help of TSPCV. It demonstrates the stability of the TSP mechanism.

Other data that should be kept for record include: volume, bus GPS record, communication record and etc.

\section{SCENARIOS DESIGN}

\section{One Bus Scenario}

The scenario evaluates the performance of TSPCV dealing with one transit vehicle at an isolated intersection. The TSPCV will be compared against the Conventional TSP (CTSP) which is simple green extension. After the data is collected, the MOEs should be compared based on different traffic conditions (time of the day, day of the week) and various arrival types (beginning of red, in the middle of the red, end of the red). It is recommended a minimum of 10 data should be collected for each arrival type and traffic condition in order to demonstrate the improvement generated by TSPCV is statistically significant.

\section{Multiple Conflicting Requests}

Three different scenarios will be tested: 1, two requests coming from perpendicular directions; 2, two requests coming from opposite directions; 3 , three conflicting requests coming from three directions. All the experiment will be conducted on one single intersection.

The TSPCVM will be compared against the conventional TSP (first come first serve). Again, after the data is collected, the MOEs should be compared based on different traffic conditions (time of the day, day of the week) and various arrival types (beginning of red, in the middle of the red, end of the red). Depends on the number of buses take participants in this experiment, the likelihood of observing multiple conflicting requests varies. If the number of experimental buses is limited, the researcher proposes to validate the benefit of TSPCV firstly based on results from "one bus scenario" and "bus progression" scenario. After these results confirm that TSPCV does save bus travel time and cause no adverse effect, the second step of this research could consider including more buses and collecting data for a longer period of time in order to observe and evaluate TSPCVM resolving multiple conflicting requests.

\section{Bus Progression}

This scenario evaluates the progression of one TSP bus travelling along two consecutive intersections. Three different TSP logic types will be compared: GTSPCV, LTSPCV and CTSP. Again, after the data is collected, the MOEs should be compared based on different traffic conditions (time of the day, day of the week) and various arrival types (beginning of red, in the middle of the red, end of the red). 


\section{DEVICES}

\section{Communication Devices}

The communication devices enable the data exchange process among vehicles, transit buses, signal and other infrastructures. These devices create a channel to provide the central control with the states of different involved parties and also send back driving instructions to the transit buses. Two candidate technologies are presented in the following: Dedicated Short Range Communication (DSRC) technology and commercial cellular services.

\section{DSRC Technology}

Dedicated Short Range Communication (DSRC) technology was developed specifically for Connected Vehicle initiatives. It refers to communications on a dedicated $5.9 \mathrm{GHz}$ frequency band reserved using the Wireless Access in Vehicular Environments (WAVE) protocols defined in the IEEE 1609 standard and its subsidiary parts. It is discovered that DSRC is better suited for applications requiring high bandwidth and low latency. One major limit of DSRC is its communication range (less than $1500 \mathrm{ft}$ ). For the deployment of the TSPCV, sometimes transit buses would require longer communication range in order to achieve the optimum time saving. In that case, additional communication devices or further treatments are needed.

\section{Commercial Cellular Services}

With increasing coverage of the " $3 G$ " and " $4 G$ " cellular network, smart phones are now an alternative option for communication. The extensive coverage of this network makes it advantageous for applications needing continuous connectivity over long distance. While, on the other hand, the network latencies and potential connection drop makes cellular services potentially problematic for real time V2X applications.

\section{GPS}

To realize the connected vehicle environment, GPS needs to be installed on all vehicles, including buses. It collects information like vehicle speeds, positions, arrival rates, rates of acceleration and deceleration, queue lengths, and stopped time and so on.

\section{APC System}

Automatic Passenger Counting (APC) system needs to be installed on buses to provide the number of passengers on board.

\section{Blue Tooth Reader}

The blue tooth reader shall be installed on all six legs of the study site and on the segment in between two intersections of interest. It would provide the ID and time stamp a specific vehicle passing through the blue tooth reader. This information will be used to compute the total travel time. 


\section{INVOLVED PARTIES}

\section{Traffic Signal Control}

Controller cabinet needs to be upgraded with roadside equipment (RSE) to enable V2X communication. The roadside equipment could either be based on DSRC technology or cellular network. As mention before, each technology has its strength and weaknesses. In case cellular network is adopted, the effect caused by network latencies and potential connection drop needs to be tested and quantified. In case DSRC technology is deployed, one can consider equip one single controller with multiple RSEs to enable detection at further locations and pass back data through closer located RSEs. As a result, larger distance can be covered by DSRC devices.

A computing engine needs to be placed at the signal control. Therefore, when data and information is collected and gathered to the signal control, a computation will be performed on site. Signal plans and suggested speeds for buses are generated and passed on to the interested parties.

\section{Transit Buses}

Transit buses require being equipped with communication devices, GPS and APC system. During the Transit Signal Priority (TSP) activation, GPS provides the control with states of the buses, including speed and location, while APC system reports the number of passengers on board. This information is transmitted to the control via the communication devices. And then, after the computation is completed at the control, advised speed will also be presented to bus drivers through the communication devices.

When commercially deployed, a display needs to be installed on the bus as well. User interface should be developed to demonstrate advisory speed to the driver. For the purpose of the field test, the advisory speed can be provided to the driver by communicating through walkie talkies.

\section{Other Motorists}

As the roll out the connected vehicle technology, it is expected that vehicles will be equipped with DSRC devices. It enables better estimation and forecast on traffic state, like arrival rates, queue lengths, and stopped time and so on. However, this developed TSPCV technique can work fairly well without the installation of DSRC. In that case, loop detector is required to be deployed on every approach of the intersections of interest. Hence, only arrival rates can be collected and other data needed will be estimation based on arrival rate. 


\section{CONCLUSIONS AND FUTURE RESEARCH}

The value and importance of providing preferences to transit buses at the signalized intersections have long been recognized by transport agencies and DOTs. However, the concerns of the uncertainty in TSP's performance and possible adverse effects associated with implementing TSP have been holding back the extensive deployment of this particular technique. In light of this situation, this research advanced the mechanism of TSP logic with a collection of techniques that can grant TSP to buses with more accuracy, better effectiveness and higher reliability while causing little adverse effect to other traffic users.

A system of TSP techniques has been invented under the Connected Vehicle environment. The first stage of the research addressed the problem of providing TSP green to single bus traveling through an isolated intersection. It laid the foundation for all the subsequent research and is embedded with the three core innovative concepts of this collection of bus priority strategies. The first concept is the idea of green time reallocation. Instead of adding additional green time to the original timing plan the proposed TSP logic splits the original green time and moves part of it to when green time is mostly needed by a transit bus. Since the green time moved is fully used on discharging vehicles, the time wasted is mostly avoided and the adverse effect associated is minimized as well. At the same time, since the TSP green time is able to start mostly any time, much greater portion of buses can take advantage of the TSP mechanism. The second concept is the idea that buses could cooperate with signal to perform TSP. By having bus varying its speed/arrival time, the flexibility of the TSP strategies is further improved. Buses are now able to avoid arriving during all red phases or during minimum green for other approaches. Furthermore, the flexibility of bus arrival time also enables the TSP green to start when the least person delay at the intersection is to be observed. This improvement ensures that almost every single bus is capable of being granted with TSP. However, they are not always receiving TSP treatment. It brings up the last but the most important concept that all the TSP is conditional on person delay of all traffic users. This criterion represents a balance of interest between the public transportation users and private vehicles. It also prevents the implementation of TSP from causing adverse effect on other traffic users. All together, the logic is developed for the base TSP granting condition in order to achieve the maximum bus fleet coverage, maximum bus delay reduction and least delay for all motorists. The benefit of TSPCV is firstly proven via a theoretical deterministic analysis. It is demonstrated that TSPCV outperforms CTSP by up to $89.5 \%$. As expected, TSPCV reduces the most bus delay during low volume condition and benefits become less as volume increases. But even at $\mathrm{v} / \mathrm{c}=1.0$ condition, the TSPCV still show benefit over CTSP by about $47 \%$. For all v/c conditions evaluated, no adverse effect is discovered. The findings from the theoretical analysis are confirmed via simulation-based evaluation. Again, four different v/c ratios are tested, which are $0.5,0.7,0.9$ and 1.0. The results show that the proposed TSP logic reduces bus delay between $9 \%$ and $84 \%$ compared to conventional TSP and between $36 \%$ and $88 \%$ compared to the no TSP condition. Both theoretical analysis and simulation evaluation show that no significant negative effects are caused by the proposed TSP logic. 
The second stage of this research developed a logic that resolves conflicting TSP requests at an isolated intersection. It is built upon the foundation of the TSPCV logic developed in the previous stage and inherits all three innovative concepts. In addition, the logic incorporates an algorithm that prioritizes the buses coming from different approaches and solves for a best signal timing that minimizes the total delay at the intersection. By determining the total number and sequence of the buses accommodated, the most bus delay is saved while, at the same time, the total delay of all motorists is minimized. The enhanced logic is evaluated using a theoretical deterministic computation and simulation-based evaluation. Three conflicting scenarios are tested: two conflicting requests from opposite directions, two conflicting requests from perpendicular directions and three conflicting requests from three directions. The theoretical computation and simulation-based evaluation show consistent results. TSPCVM outperforms CTSP and reduce bus delay between 5\% and 48\%. The range of improvement corresponding to the four different $\mathrm{v} / \mathrm{c}$ ratios tested, which are $0.5,0.7,0.9$ and 1.0 , respectively. Delay reduction increases as $\mathrm{v} / \mathrm{c}$ ratio decreases. It is discovered that TSPCVM generates more benefit when buses are coming from opposite directions. Not only the bus delay is reduced more, delay per person is also minimized in a greater magnitude. The least improvement is observed when accommodating three conflicting requests. Both theoretical analysis and simulation evaluation show that no significant negative effects are caused by the proposed TSP logic.

The third stage of this research solves the problem of maintaining bus progression along a corridor of interest. Again, it is built upon the foundation of the TSPCV logic developed in the previous stage and inherits all three innovative concepts. In this case, the algorithm embedded optimizes the signal time of all intersections along the corridor as a whole. It ensures that the bus granted with TSP is able to travel through the entire corridor without stopping, and at the same time, maintains the least total delay of all motorists. This mechanism prevents the benefit buses receive at one intersection from losing to the next, with the precondition that no adverse effect is caused. Theoretical analysis reveals that, without the consideration of the bus progression, the previously developed TSPCV mechanism is able to generate sizable benefit when the spacing between signals is large. But this benefit declines greatly as the spacing narrows. GTSPCV always demonstrates superior improvement than the other two treatments for the spacing cases considered in this study. The benefit of GTSPCV grows slightly with the intersection spacing, but it does not always necessarily maintain a positive correlation. Sensitivity study on congestion level is performed for GTSPCV. Four different v/c ratios are tested, which are 0.5, 0.7, 0.9 and 1.0. Results show that the GTSPCV logic reduces the bus delay between $35 \%$ and $68 \%$ compared to the conventional TSP. The results also show that, for congestion levels below capacity, GTSPCV causes no adverse effect. Although little adverse effects on side streets are observed when the volume reaches capacity, the total delay increase is very minor. The magnitude of this increase is less than 1 second per person.

Finally, the research also presents a technique that it considers the help buses can use to merge back into the traffic more efficiently from a nearside bus stop. The research provides theoretical 
support for the value of adding queue jumper function to near-side bus stops. It is discovered that queue jumper bus stop with $\mathrm{CV}$ technology is the most recommended way of adding queue jumper lane to near-side bus stop. This mechanism could reduce up to $30 \%$ delay for buses. As the combination of all four bus priority strategies, this system of TSP technology is now a complete architecture covering most of the scenarios that buses could possibly encounter on a road network.

The value of the proposed TSP techniques is showing in many different aspects. First of all, by implementing these techniques, a much higher percentage of transit buses are able to benefit from the TSP mechanism which will eventually lead to an improved transit service with less delay, higher mobility and nicer quality of service. Secondly, the techniques also relieve the agencies and DOTs from the aforementioned major concern that TSP interrupts the progression on side streets and cause tremendous delay on other traffic users. Hence, this feature allows DOTs to save cost for not performing a study of LOS and/or V/C ratio for every potential TSP intersection before installation. Since local agencies and DOTs do not need to validate potential TSP intersections for adverse effect before installation, the proposed TSP techniques could save cost on installation and maintenance. One can expect that, with this newly developed TSP system, more intersection will be installed with TSP, better riding experience will be achieved, and higher transit ridership will be observed. All these changes will lead to a mode switch from private vehicles to public transportations and eventually lighten the burden on the existing road network and improve the traffic condition. Finally, the proposed technique is one of the few ITS applications that is capable of being realized in the early stage of the ITS roll-out. The aforementioned concept is made possible by Connected Vehicle technology which provides twoway communications and additional and more accurate information. In order to achieve the best performance of this system of TSP technology, it is preferred that all traffic users are equipped with CV technology. Nevertheless, unlike most of the other Intelligent Transportation System (ITS) applications, the proposed TSP techniques can produce sizable benefit even when only buses are equipped. This feature makes the proposed TSP techniques a good start point to promote ITS system at the early stage of ITS technology deployment.

Future research could consider consolidate all the techniques into an ultimate TSP logic. This TSP will be able to accommodate multiple conflicting TSP requests for buses traveling on a road network. This scenario would become more complicated as the maintaining of bus progression will cause significantly more complications than the isolated intersection scenario. Apart from that, additional condition criteria could also be tested instead of per person delay, for example, fuel consumption, emission, etc. In addition, investigation concerning the field implementation of the proposed TSP techniques is worth performing as well. 


\section{REFERENCES}

1. Zlatkovic, M., A. Stevanovic, and P. T. Martin. Development and Evaluation of Algorithm for Resolution of Conflicting Transit Signal Priority Requests. Transportation Research Record: Journal of the Transportation Research Board, 2012, pp. 167-175.

2. Hill, C. J., and J. K. Garrett. AASHTO Connected Vehicle Infrastructure Deployment Analysis. FHWA-JPO-11-090, , 2011.

3. Updated Descriptions on USDOT High-Priority Dynamic Mobility Applications. http://www.its.dot.gov/press/2011/mobility app.htm, Accessed April 18, 2013.

4. Volpe, J. A. Vehicle-Infrastructure Integration (VII) Initiative Benefit-Cost Analysis Version 2.3 . Federal Highway Administration, 2008. 